\title{
ON A CLASS OF NONLINEAR SCHRÖDINGER-POISSON SYSTEMS INVOLVING A NONRADIAL CHARGE DENSITY
}

\author{
CARLO MERCURI AND TERESA MEGAN TYLER
} \begin{abstract}
Schrödinger-Poisson system

$$
\begin{cases}-\Delta u+u+\rho(x) \phi u=|u|^{p-1} u, & x \in \mathbb{R}^{3}, \\ -\Delta \phi=\rho(x) u^{2}, & x \in \mathbb{R}^{3},\end{cases}
$$
\end{abstract}

Abstract. In the spirit of the classical work of P. H. Rabinowitz on nonlinear Schrödinger equations, we prove existence of mountain-pass solutions and least energy solutions to the nonlinear

under different assumptions on $\rho: \mathbb{R}^{3} \rightarrow \mathbb{R}_{+}$at infinity. Our results cover the range $p \in(2,3)$ where the lack of compactness phenomena may be due to the combined effect of the invariance by translations of a 'limiting problem' at infinity and of the possible unboundedness of the PalaisSmale sequences. Moreover, we find necessary conditions for concentration at points to occur for solutions to the singularly perturbed problem

$$
\begin{cases}-\epsilon^{2} \Delta u+u+\rho(x) \phi u=|u|^{p-1} u, & x \in \mathbb{R}^{3}, \\ -\Delta \phi=\rho(x) u^{2}, & x \in \mathbb{R}^{3},\end{cases}
$$

in various functional settings which are suitable for both variational and perturbation methods. MSC: 35J20, 35B65, 35J60, 35Q55

Keywords: Stationary Nonlinear Schrödinger-Poisson System, Weighted Sobolev Spaces, Palais-Smale Sequences, Lack of Compactness.

\section{CONTEnts}

1. Introduction 2

1.1. Main results 4

1.2. Related questions 11

1.3. Outline 12

1.4. Notation 12

Acknowledgements 13

2. Preliminaries 13

2.1. The space $E\left(\mathbb{R}^{3}\right) \quad 13$

2.2. Regularity and positivity 13

2.3. Pohozaev identity 14

3. The min-max setting: definition of $c_{\mu}, c_{\mu}^{\infty}, c$, and $c^{\infty} \quad 14$

3.1. Lower bounds for $I$ and $I^{\infty} \quad 18$

4. Existence: the case of coercive $\rho(x) \quad 20$

4.1. Proof of Theorem $2 \quad 21$

4.2. Proof of Corollary $1 \quad 24$

5. Existence: the case of non-coercive $\rho(x) \quad 25$

5.1. $\quad$ Bounded PS sequences: proof of Proposition $2 \quad 25$

5.2. Proof of Theorem $3 \quad 35$

5.3. Proof of Theorem $4 \quad 37$

5.4. Proof of Corollary $2 \quad 38$

6. Necessary conditions for concentration of semiclassical states 39 
6.1. Proof of Theorem $5 \quad 39$

6.2. Proof of Theorem $6 \quad 47$

Appendix: proof of Lemma $2.2 \quad 47$

References $\quad 50$

\section{INTRODUCTION}

We study existence of positive solutions to the following nonlinear Schrödinger-Poisson system

$$
\begin{cases}-\Delta u+u+\rho(x) \phi u=|u|^{p-1} u, & x \in \mathbb{R}^{3} \\ -\Delta \phi=\rho(x) u^{2}, & x \in \mathbb{R}^{3}\end{cases}
$$

with $p \in(2,5)$ and $\rho: \mathbb{R}^{3} \rightarrow \mathbb{R}$ a nonnegative measurable function which represents a non-constant 'charge' corrector to the density $u^{2}$. In the context of the so-called Density Functional Theory, variants of system (1.1) appear as mean field approximations of quantum many-body systems, see [6], [18], [37]. The positive Coulombic potential, $\phi$, represents a repulsive interaction between particles, whereas the local nonlinearity $|u|^{p-1} u$ generalises the $u^{5 / 3}$ term introduced by Slater [46] as local approximation of the exchange potential in Hartree-Fock type models, see e.g. [12], [38].

Within a min-max setting and in the spirit of Rabinowitz [43], we study existence and qualitative properties of the solutions to (1.1), highlighting those phenomena which are driven by $\rho$. The system (1.1) 'interpolates' the classical equation

$$
-\Delta u+u=u^{p}, \quad x \in \mathbb{R}^{3},
$$

whose positive solutions have been classified by Kwong [33], with

$$
\begin{cases}-\Delta u+u+\phi u=|u|^{p-1} u, & x \in \mathbb{R}^{3} \\ -\Delta \phi=u^{2}, & x \in \mathbb{R}^{3}\end{cases}
$$

studied by several authors in relation to existence, nonexistence, multiplicity and behaviour of the solutions in the semi-classical limit; see e.g. [2], [10], [18], and references therein. In the case $\rho(x) \rightarrow 0$ as $|x| \rightarrow+\infty$, (1.2) has been exploited as limiting equation to tackle existence/compactness questions related to certain classes of systems similar to (1.1), see e.g. [20] and [21]. In the present paper we consider instances where the convergence of approximating solutions to (1.1) is not characterised by means of (1.2), namely the cases where, as $|x| \rightarrow+\infty$, it holds either that $\rho \rightarrow+\infty$ ('coercive case'), or that $\rho \rightarrow \rho_{\infty}>0$ ('non-coercive case'). The latter corresponds to the case where nontrivial solutions of (1.3) (up to coefficients) cause lack of compactness phenomena to occur. The main difficulty in this context is that, despite the extensive literature, a full understanding of the set of positive solutions to (1.3) has not yet been achieved (symmetry, non-degeneracy, etc.).

The autonomous system (1.3), as well as (1.1), presents various mathematical features which are not shared with nonlinear Schrödinger type equations, mostly related to lack of compactness phenomena. In a pioneering work [45], radial functions and constrained minimisation techniques have been used, over a certain natural constraint manifold defined combining the Pohozaev and 
Nehari identities, yielding existence results of positive radial solutions to (1.3) for all $p \in(2,5)$. Again in a radial setting, a variant of system (1.3) has been studied more recently in [32]. When $p \leq 2$ the change in geometry of the associated energy functional causes differing phenomena to occur. In [45] existence, nonexistence and multiplicity results have been shown to be sensitive to a multiplicative factor for the Poisson term. Nonexistence results for (1.3) have also been obtained in $\mathbb{R}^{3}$ in the range $p \geq 5$ (see e.g. [22]). In the presence of potentials, however, existence may occur when $p=5$, as it has been recently shown in [19]. Ambrosetti and Ruiz [5] improved upon these early results by using the so-called 'monotonicity trick' introduced by Struwe [47] and formulated in the context of the nonlinear Schrödinger equations by Jeanjean [30] and Jeanjean-Tanaka [31], in order to show the existence of multiple bound state solutions to (1.3).

Related problems involving a non-constant charge density $\rho$, and in the presence of potentials, have been studied. The vast majority of works involve the range $p>3$ since, when $p \leq 3$, one has to face two major obstacles in applying the minimax methods: constructing bounded Palais-Smale sequences and proving that the Palais-Smale condition holds, see e.g [45], and [5], [39]. Cerami and Molle [20] and Cerami and Vaira [21] studied the system

$$
\begin{cases}-\Delta u+V(x) u+\lambda \rho(x) \phi u=K(x)|u|^{p-1} u, & x \in \mathbb{R}^{3}, \\ -\Delta \phi=\rho(x) u^{2}, & x \in \mathbb{R}^{3},\end{cases}
$$

where $\lambda>0$ and $V(x), \rho(x)$ and $K(x)$ are nonnegative functions in $\mathbb{R}^{3}$ such that

$$
\lim _{|x| \rightarrow+\infty} \rho(x)=0, \quad \lim _{|x| \rightarrow+\infty} V(x)=V_{\infty}>0, \quad \lim _{|x| \rightarrow+\infty} K(x)=K_{\infty}>0
$$

and, under suitable assumptions on the potentials, proved the existence of positive ground state and bound state solutions for $p \in(3,5)$. In [15] existence of positive solutions to (1.4) in the range $p \in[3,5)$ has been proved under suitable assumptions on the potentials that guarantee some compact embeddings of weighted Sobolev spaces into weighted $L^{p+1}$ spaces. Vaira [50] also studied system (1.4), in the case that

$$
\lim _{|x| \rightarrow+\infty} \rho(x)=\rho_{\infty}>0, V(x) \equiv 1, \lim _{|x| \rightarrow+\infty} K(x)=K_{\infty}>0
$$

and, assuming $\lambda>0$ and $K(x) \not \equiv 1$, proved the existence of positive ground state solutions for $p \in(3,5)$. In a recent and interesting paper, Sun, Wu and Feng (see Theorem 1.4 of [49]) have shown the existence of a solution to (1.4) for $p \in(1,3]$, assuming (1.6) and $K(x) \equiv 1$, provided $\lambda$ is sufficiently small and $\int_{\mathbb{R}^{3}} \rho(x) \phi_{\rho, w_{\lambda}} w_{\lambda}^{2}<\int_{\mathbb{R}^{3}} \rho_{\infty} \phi_{\rho_{\infty}, w_{\lambda}} w_{\lambda}^{2}$, where $\left(w_{\lambda}, \phi_{\rho_{\infty}, w_{\lambda}}\right)$ is a positive solution to

$$
\begin{cases}-\Delta u+u+\lambda \rho_{\infty} \phi u=|u|^{p-1} u, & x \in \mathbb{R}^{3} \\ -\Delta \phi=\rho_{\infty} u^{2}, & x \in \mathbb{R}^{3}\end{cases}
$$

Their results are obtained using the fact that all nontrivial solutions to (1.4) lie in a certain manifold $M_{\lambda}^{-}$(see Lemma 6.1 in [49]) to show that the energy functional $J_{\lambda}$ is bounded from below on the set of nontrivial solutions to (1.4). We believe that this is necessary to prove Corollary 4.3 in [49], and, ultimately, to prove Theorem 1.4 in [49], and thus the existence result is only viable in the reduced range $2.18 \approx \frac{-2+\sqrt{73}}{3}<p \leq 3$ and provided the additional assumption $\frac{3 p^{2}+4 p-23}{2(5-p)} \rho(x)+\frac{p-1}{2}(\nabla \rho(x), x) \geq 0$ also holds. In this range of $p$ and under these assumptions, as observed in [49], solutions are ground states. 
1.1. Main results. In light of the above results, we aim to study existence and qualitative properties of the solutions to (1.1), in the various functional settings corresponding to different hypotheses on the behaviour of $\rho$ at infinity. Throughout the paper we set $D^{1,2}\left(\mathbb{R}^{3}\right)=D^{1,2}$ as the space defined as

$$
D^{1,2}\left(\mathbb{R}^{3}\right):=\left\{u \in L^{6}\left(\mathbb{R}^{3}\right): \nabla u \in L^{2}\left(\mathbb{R}^{3}\right)\right\}
$$

and equipped with norm

$$
\|u\|_{D^{1,2}\left(\mathbb{R}^{3}\right)}:=\|\nabla u\|_{L^{2}\left(\mathbb{R}^{3}\right)} .
$$

It is well-known that if $u^{2} \rho \in L_{\text {loc }}^{1}\left(\mathbb{R}^{3}\right)$ is such that

$$
\int_{\mathbb{R}^{3}} \int_{\mathbb{R}^{3}} \frac{u^{2}(x) \rho(x) u^{2}(y) \rho(y)}{|x-y|} \mathrm{d} x \mathrm{~d} y<+\infty
$$

then,

$$
\phi_{u}(x):=\int_{\mathbb{R}^{3}} \frac{\rho(y) u^{2}(y)}{\omega|x-y|} \mathrm{d} y \in D^{1,2}\left(\mathbb{R}^{3}\right)
$$

is the unique weak solution in $D^{1,2}\left(\mathbb{R}^{3}\right)$ of the Poisson equation

$$
-\Delta \phi=\rho(x) u^{2}
$$

and it holds that

$$
\int_{\mathbb{R}^{3}}\left|\nabla \phi_{u}\right|^{2}=\int_{\mathbb{R}^{3}} \rho \phi_{u} u^{2} \mathrm{~d} x=\int_{\mathbb{R}^{3}} \int_{\mathbb{R}^{3}} \frac{u^{2}(x) \rho(x) u^{2}(y) \rho(y)}{\omega|x-y|} \mathrm{d} x \mathrm{~d} y .
$$

Here, $\omega=4 \pi$. Using the explicit representation of $\phi_{u}$ the system (1.1) reduces to solving the problem

$$
-\Delta u+u+\rho(x) \phi_{u} u=|u|^{p-1} u,
$$

whose positive solutions are critical points of the functional

$$
I(u):=\frac{1}{2} \int_{\mathbb{R}^{3}}\left(|\nabla u|^{2}+u^{2}\right)+\frac{1}{4} \int_{\mathbb{R}^{3}} \rho \phi_{u} u^{2}-\frac{1}{p+1} \int_{\mathbb{R}^{3}} u_{+}^{p+1},
$$

which is natural to define in $E\left(\mathbb{R}^{3}\right) \subseteq H^{1}\left(\mathbb{R}^{3}\right)$

where

$$
E\left(\mathbb{R}^{3}\right):=\left\{u \in D^{1,2}\left(\mathbb{R}^{3}\right):\|u\|_{E}<+\infty\right\},
$$

$$
\|u\|_{E}^{2}:=\int_{\mathbb{R}^{3}}\left(|\nabla u|^{2}+u^{2}\right) \mathrm{d} x+\left(\int_{\mathbb{R}^{3}} \int_{\mathbb{R}^{3}} \frac{u^{2}(x) \rho(x) u^{2}(y) \rho(y)}{|x-y|} \mathrm{d} x \mathrm{~d} y\right)^{1 / 2} .
$$


Variants of this space have been studied since the work of P.L. Lions [35], see e.g. [44], and [8],[15], [40]. We recall that by the classical Hardy-Littlewood-Sobolev inequality, it holds that

$$
\left|\int_{\mathbb{R}^{3}} \int_{\mathbb{R}^{3}} \frac{u^{2}(x) \rho(x) u^{2}(y) \rho(y)}{\omega|x-y|} \mathrm{d} x \mathrm{~d} y\right| \leq C|| \rho u^{2} \|_{L^{\frac{6}{5}}\left(\mathbb{R}^{3}\right)}^{2},
$$

for some $C>0$. Thus, if $u \in H^{1}\left(\mathbb{R}^{3}\right)$, we see that, depending on the assumptions on $\rho$, we may not be able to control the Coulomb integral (1.8) using the natural bound provided by HLS inequality. This may be the case if e. g. $\rho(x) \rightarrow+\infty$ as $|x| \rightarrow+\infty$. For these reasons, in all of the results of the present paper we also analyse those instances where $E\left(\mathbb{R}^{3}\right)$ and $H^{1}\left(\mathbb{R}^{3}\right)$ do not coincide.

As a warm-up observation we have the following theorem regarding existence in the coercive case for $p \geq 3$.

Theorem 1. [Coercive case: existence of mountain pass solution for $p \geq 3$ ] Suppose $\rho \in C\left(\mathbb{R}^{3}\right)$ is nonnegative and $\rho(x) \rightarrow+\infty$ as $|x| \rightarrow+\infty$. Then, for any $p \in[3,5)$, there exists a solution, $\left(u, \phi_{u}\right) \in E\left(\mathbb{R}^{3}\right) \times D^{1,2}\left(\mathbb{R}^{3}\right)$, of $(1.1)$, whose components are positive functions. In particular, $u$ is a mountain pass critical point of I at level $c$, where $c$ is the min-max level defined in (3.7).

Proof. Since $\rho(x) \rightarrow+\infty$ as $|x| \rightarrow+\infty$, then $E$ is compactly embedded in $L^{p+1}\left(\mathbb{R}^{3}\right)$ by Lemma 4.1 below, and therefore the existence of a Mountain Pass solution $u$ to (1.1) is provided by Theorem 1 of [15]. Both $u, \phi_{u}$ are positive by the strong maximum principle, and this concludes the proof.

It is also worth finding conditions such that the term $\rho u^{2}$ goes to zero at infinity, since the whole right hand side of the Poisson equation is classically interpreted as a 'charge density'. This is provided by the following.

Proposition 1. [Decay of $u$ and $\left.\rho u^{2}\right]$ Let $\rho: \mathbb{R}^{3} \rightarrow \mathbb{R}$ be continuous and nonnegative, $p \in[1,5]$, and $\left(u, \phi_{u}\right) \in E\left(\mathbb{R}^{3}\right) \times D^{1,2}\left(\mathbb{R}^{3}\right)$ be solution to (1.1). Assume that $u$ is nonnegative. Then, for every $\gamma \in(0,1)$, there exists $C>0$ such that

$$
u(x) \leq C e^{-\gamma(1+|x|)} \quad\left(L^{2} \text {-decay }\right) .
$$

If, in addition, $\rho$ is such that

(i) $\liminf _{|x| \rightarrow \infty} \rho(x)|x|^{1-2 \alpha}>A$

(ii) $\lim \sup _{|x| \rightarrow \infty} \rho(x) e^{-\beta(1+|x|)^{\alpha}} \leq B$

for some $\alpha, \beta, A, B>0$, with $\beta<2 \sqrt{A}$, then, for some constant $C>0$, it holds that

(a) $u(x) \leq C e^{-\sqrt{A}(1+|x|)^{\alpha}}$ 
and therefore

(b) $\rho(x) u^{2}(x)=O\left(e^{(\beta-2 \sqrt{A})(1+|x|)^{\alpha}}\right), \quad$ as $|x| \rightarrow+\infty$.

Proof. The conclusion easily follows by Theorem 6 in [15] (see also [16]). More precisely, setting $W(x):=1+\frac{\rho(x)}{|x|}$, the $L^{2}$-decay follows as $W(x) \geq 1$ and therefore

$$
\liminf _{|x| \rightarrow+\infty} W(x)>\gamma^{2}
$$

is automatically satisfied for every $\gamma \in(0,1)$. Moreover, note that by $(i)$ it follows that

$$
\liminf _{|x| \rightarrow+\infty} W(x)|x|^{2-2 \alpha} \geq \liminf _{|x| \rightarrow+\infty} \rho(x)|x|^{1-2 \alpha}>A
$$

which yields $(a)$ again by Theorem 6 in [15]. This concludes the proof.

With these preliminary results in place, we first study the case of coercive $\rho$, namely $\rho(x) \rightarrow+\infty$ as $|x| \rightarrow+\infty$, and work in the natural setting for this problem, $E\left(\mathbb{R}^{3}\right)$. When $p \in(2,3)$, we make use of the aforementioned 'monotonicity trick' exploiting the structure of our functional, in order to construct bounded Palais-Smale sequences for small perturbations of (1.1). We are able to prove that these sequences converge using a compact embedding established in Lemma 4.1. We finally show that these results extend to the original problem and obtain the following theorem.

Theorem 2. [Coercive case: existence of mountain pass solution for $p \in(2,3)]$ Suppose $\rho \in C\left(\mathbb{R}^{3}\right) \cap W_{\text {loc }}^{1,1}\left(\mathbb{R}^{3}\right)$ is nonnegative and $\rho(x) \rightarrow+\infty$ as $|x| \rightarrow+\infty$. Suppose further that $k \rho(x) \leq(x, \nabla \rho)$ for some $k>\frac{-2(p-2)}{(p-1)}$. Then, for any $p \in(2,3)$, there exists a solution, $\left(u, \phi_{u}\right) \in E\left(\mathbb{R}^{3}\right) \times D^{1,2}\left(\mathbb{R}^{3}\right)$, of (1.1), whose components are positive functions. In particular, $u$ is a mountain pass critical point of I at level $c$, where $c$ is the min-max level defined in (3.7).

After establishing these results, we prove the existence of least energy solutions for all $p \in(2,5)$. It is important to note that for $p \in(3,5)$ the solutions provided by the following corollary coincide with those provided by Theorem 1 . For $p \in(2,3]$, we make use of a minimising sequence in order to obtain the result, however we do not know whether the least energy solutions provided by what follows are the same as those provided by Theorem $1(p=3)$ and Theorem 2 .

Corollary 1. [Coercive case: existence of a least energy solution for $p \in(2,5)]$ Suppose $\rho \in C\left(\mathbb{R}^{3}\right)$ is nonnegative and $\rho(x) \rightarrow+\infty$ as $|x| \rightarrow+\infty$. If $p \in(2,3)$, suppose, in addition, that $\rho \in W_{\text {loc }}^{1,1}\left(\mathbb{R}^{3}\right)$ and $k \rho(x) \leq(x, \nabla \rho)$ for some $k>\frac{-2(p-2)}{(p-1)}$. Then, for all $p \in(2,5)$, there exists a solution, $\left(u, \phi_{u}\right) \in E\left(\mathbb{R}^{3}\right) \times D^{1,2}\left(\mathbb{R}^{3}\right)$, of $(1.1)$, whose components are positive functions, such that $u$ is a least energy critical point of $I$.

Remark 1. If we define

$$
\mathcal{I}(u):=\frac{1}{2} \int_{\mathbb{R}^{3}}\left(|\nabla u|^{2}+u^{2}\right)+\frac{1}{4} \int_{\mathbb{R}^{3}} \rho \phi_{u} u^{2}-\frac{1}{p+1} \int_{\mathbb{R}^{3}}|u|^{p+1},
$$


then, under the same assumptions on $\rho$ as in Corollary 1, we can prove the existence of a least energy critical point for $\mathcal{I}$ for all $p \in(2,5)$ by following similar techniques to those used in the proof of Corollary 1. Since for $p>3$ the mountain pass level is equal to the infimum on the Nehari manifold, in this range it is possible to select a positive groundstate critical point for $\mathcal{I}$. It is not clear whether this is also the case for $p \in(2,3]$.

We then focus on the case of non-coercive $\rho$, namely when $\rho(x) \rightarrow \rho_{\infty}>0$ as $|x| \rightarrow+\infty$. For this problem, $E\left(\mathbb{R}^{3}\right)$ coincides with the larger space $H^{1}\left(\mathbb{R}^{3}\right)$, and so we look for solutions $\left(u, \phi_{u}\right) \in H^{1}\left(\mathbb{R}^{3}\right) \times D^{1,2}\left(\mathbb{R}^{3}\right)$ of (1.1). Our method relies on an a posteriori compactness analysis of bounded Palais-Smale sequences (in the spirit of the classical book of M. Willem [52]), in which we find that any possible lack of compactness is related to the invariance by translations of the subcritical 'problem at infinity' associated to (3.1), namely

$$
-\Delta u+u+\rho_{\infty} \bar{\phi}_{u} u=|u|^{p-1} u
$$

where $\bar{\phi}_{u}(x):=\int_{\mathbb{R}^{3}} \frac{\rho_{\infty} u^{2}(y)}{\omega|x-y|} \mathrm{d} y \in D^{1,2}\left(\mathbb{R}^{3}\right)$. Positive solutions of (1.13) are critical points of the corresponding functional, $I^{\infty}: H^{1}\left(\mathbb{R}^{3}\right) \rightarrow \mathbb{R}$, defined as

$$
I^{\infty}(u):=\frac{1}{2} \int_{\mathbb{R}^{3}}\left(|\nabla u|^{2}+u^{2}\right)+\frac{1}{4} \int_{\mathbb{R}^{3}} \rho_{\infty} \bar{\phi}_{u} u^{2}-\frac{1}{p+1} \int_{\mathbb{R}^{3}} u_{+}^{p+1}
$$

When $p \in(2,3)$, we define perturbations of $I$ and $I^{\infty}$, namely $I_{\mu}$ and $I_{\mu}^{\infty}$ (see Section 3), as follows

$$
I_{\mu}(u):=\frac{1}{2} \int_{\mathbb{R}^{3}}\left(|\nabla u|^{2}+u^{2}\right)+\frac{1}{4} \int_{\mathbb{R}^{3}} \rho \phi_{u} u^{2}-\frac{\mu}{p+1} \int_{\mathbb{R}^{3}} u_{+}^{p+1}
$$

and

$$
I_{\mu}^{\infty}(u):=\frac{1}{2} \int_{\mathbb{R}^{3}}\left(|\nabla u|^{2}+u^{2}\right)+\frac{1}{4} \int_{\mathbb{R}^{3}} \rho_{\infty} \bar{\phi}_{u} u^{2}-\frac{\mu}{p+1} \int_{\mathbb{R}^{3}} u_{+}^{p+1}
$$

The aforementioned a posteriori compactness analysis is provided by the following proposition. There are several compactness results of similar flavour since the pioneering works of P.L. Lions [36] and Benci-Cerami [9], which include more recent contributions in the context of SchrödingerPoisson systems, see e.g. [21], [50], [19]. We point out that these recent results are mostly in the range $p>3$, for Palais-Smale sequences constrained on Nehari manifolds, and for functionals without positive parts, unlike our result.

Proposition 2. [Global compactness for bounded PS sequences] Suppose $\rho \in C\left(\mathbb{R}^{3}\right)$ is nonnegative and $\rho(x) \rightarrow \rho_{\infty} \geq 0$ as $|x| \rightarrow+\infty$. Let $p \in(2,5)$ and $\mu \in\left[\frac{1}{2}, 1\right]$ and assume $\left(u_{n}\right)_{n \in \mathbb{N}} \subset H^{1}\left(\mathbb{R}^{3}\right)$ is a bounded Palais-Smale sequence for $I_{\mu}$. Then, there exists $l \in \mathbb{N}$, a finite sequence $\left(v_{0}, \ldots, v_{l}\right) \subset H^{1}\left(\mathbb{R}^{3}\right)$, and $l$ sequences of points $\left(y_{n}^{j}\right)_{n \in \mathbb{N}} \subset \mathbb{R}^{3}, 1 \leq j \leq l$, satisfying, up to a subsequence of $\left(u_{n}\right)_{n \in \mathbb{N}}$,

(i) $v_{0}$ is a nonnegative solution of (3.1), 
(ii) $v_{j}$ are nonnegative, and possibly nontrivial, solutions of (3.8) for $1 \leq j \leq l$,

(iii) $\left|y_{n}^{j}\right| \rightarrow+\infty,\left|y_{n}^{j}-y_{n}^{j^{\prime}}\right| \rightarrow+\infty$ as $n \rightarrow+\infty$ if $j \neq j^{\prime}$,

(iv) $\left\|u_{n}-v_{0}-\sum_{j=1}^{l} v_{j}\left(\cdot-y_{n}^{j}\right)\right\|_{H^{1}\left(\mathbb{R}^{3}\right)} \rightarrow 0$ as $n \rightarrow+\infty$,

(v) $\left\|u_{n}\right\|_{H^{1}\left(\mathbb{R}^{3}\right)}^{2} \rightarrow \sum_{j=0}^{l}\left\|v_{j}\right\|_{H^{1}\left(\mathbb{R}^{3}\right)}^{2}$ as $n \rightarrow+\infty$,

(vi) $I_{\mu}\left(u_{n}\right)=I_{\mu}\left(v_{0}\right)+\sum_{j=1}^{l} I_{\mu}^{\infty}\left(v_{j}\right)+o(1)$.

Remark 2. In the case $\rho_{\infty}=0$, the limiting equation (3.8) reduces to coincide with (1.2).

Roughly speaking, the presence of $u_{+}$in the functional $I_{\mu}$ and Sobolev's inequality imply that $\left(u_{n}\right)_{-} \rightarrow 0$ in $L^{p+1}$. It is therefore possible to use an observation to the classical Brezis-Lieb lemma [17] made in [42] to show that it also holds that $\left(u_{n}-v_{0}\right)_{-} \rightarrow 0$ in $L^{p+1}$. In the proof we also take advantage of recent nonlocal versions of the Brezis-Lieb lemma, see [7] and [40].

Remark 3. Dropping the + subscript in the definition of $I_{\mu}$ and simply observing that for every continuous path $\gamma:[0,1] \rightarrow H^{1}$ it holds that $I_{\mu}(\gamma(t))=I_{\mu}(|\gamma(t)|)$, a quantitative deformation argument (see e.g. Lemma 2.3 and Theorem 2.8 in [52]) would allow us, for almost every $\mu \in\left[\frac{1}{2}, 1\right]$, to construct a bounded Palais-Smale sequence $\left(u_{\mu, n}\right)_{n \in \mathbb{N}}$ for $I_{\mu}$ at the level $c_{\mu}$ (defined in (3.5)) such that

$$
\operatorname{dist}\left(u_{\mu, n}, \mathcal{P}\right) \rightarrow 0, \quad \mathcal{P}=\left\{u \in H^{1}\left(\mathbb{R}^{3}\right) \mid u_{-} \equiv 0\right\}
$$

We have opted for a less abstract approach to positivity.

In the case $p \in(2,3)$, we use Proposition 2 together with Pohozaev's and Nehari's identities to show that a sequence of approximated critical points, constructed by means of the "monotonicity trick', is relatively compact. This enables us to obtain the following result.

Theorem 3. [Non-coercive case: existence of mountain pass solution for $p \in(2,3)$ ] Suppose $\rho \in C\left(\mathbb{R}^{3}\right) \cap W_{\text {loc }}^{1,1}\left(\mathbb{R}^{3}\right)$ is nonnegative, $\rho(x) \rightarrow \rho_{\infty}>0$ as $|x| \rightarrow+\infty$, and $k \rho(x) \leq(x, \nabla \rho)$ for some $k>\frac{-2(p-2)}{(p-1)}$. Suppose further that either

(i) $c<c^{\infty}$

or

(ii) $\rho(x) \leq \rho_{\infty}$ for all $x \in \mathbb{R}^{3}$, with strict inequality, $\rho(x)<\rho_{\infty}$, on some ball $B \subset \mathbb{R}^{3}$,

where $c$ and (resp.) $c^{\infty}$ are min-max levels defined in (3.7) and (resp.) (3.11). Then, for any $p \in(2,3)$, there exists a solution, $\left(u, \phi_{u}\right) \in H^{1}\left(\mathbb{R}^{3}\right) \times D^{1,2}\left(\mathbb{R}^{3}\right)$, of $(1.1)$, whose components are positive functions. In particular, $u$ is a mountain pass critical point of $I$ at level $c$. 
The non-coercive case turns out to be more 'regular' with respect to compactness issues when $p \geq 3$. In fact, we can show that the Palais-Smale condition holds at the mountain pass level $c$ and as a consequence we have the following

Theorem 4. [Non-coercive case: existence of mountain pass solution for $p \geq 3$ ] Suppose $\rho \in C\left(\mathbb{R}^{3}\right)$ is nonnegative and $\rho(x) \rightarrow \rho_{\infty}>0$ as $|x| \rightarrow+\infty$. Let either of the following conditions hold:

(i) $c<c^{\infty}$

or

(ii) $\rho(x) \leq \rho_{\infty}$ for all $x \in \mathbb{R}^{3}$, with strict inequality, $\rho(x)<\rho_{\infty}$, on some ball $B \subset \mathbb{R}^{3}$,

where $c$ and (resp.) $c^{\infty}$ are minimax levels defined in (3.7) and (resp.) (3.11). Then, for any $p \in[3,5)$ there exists a solution, $\left(u, \phi_{u}\right) \in H^{1}\left(\mathbb{R}^{3}\right) \times D^{1,2}\left(\mathbb{R}^{3}\right)$, of $(1.1)$, whose components are positive functions. In particular, $u$ is a mountain pass critical point of $I$ at level $c$.

We follow up the previous two theorems with a result giving the existence of least energy solutions in the non-coercive case. When $p \in(3,5)$ the existence follows relatively straightforwardly using the Nehari characterisation of the mountain pass level, and when $p \in(2,3]$ we use a minimising sequence together with Proposition 2 to obtain the result.

Corollary 2. [Non-coercive case: existence of least energy solution for $p \in(2,5)]$ Suppose $\rho \in C\left(\mathbb{R}^{3}\right)$ is nonnegative, $\rho(x) \rightarrow \rho_{\infty}>0$ as $|x| \rightarrow+\infty$, and one of the following conditions hold:

(i) $c<c^{\infty}$

or

(ii) $\rho(x) \leq \rho_{\infty}$ for all $x \in \mathbb{R}^{3}$, with strict inequality, $\rho(x)<\rho_{\infty}$, on some ball $B \subset \mathbb{R}^{3}$,

where $c$ and (resp.) $c^{\infty}$ are minimax levels defined in (3.7) and (resp.) (3.11). If $p \in(2,3)$, suppose in addition that $\rho \in W_{\text {loc }}^{1,1}\left(\mathbb{R}^{3}\right)$ and $k \rho(x) \leq(x, \nabla \rho)$ for some $k>\frac{-2(p-2)}{(p-1)}$. Then, for all $p \in(2,5)$, there exists a solution, $\left(u, \phi_{u}\right) \in H^{1}\left(\mathbb{R}^{3}\right) \times D^{1,2}\left(\mathbb{R}^{3}\right)$, of $(1.1)$, whose components are positive functions, such that $u$ is a least energy critical point of $I$.

Remark 4. By following similar techniques to those used in the proof of Corollary 2, we can show that under the same assumptions as this corollary (with obvious modifications to the minimax levels), there exists a least energy solution for $\mathcal{I}$, defined in (1.12), for all $p \in(2,5)$. As in the coercive case, it is not clear if we can select a positive groundstate for $p \in(2,3]$.

After establishing these existence results, it is natural to ask if the non-locality of the equation allows us to find localised solutions. Moreover, we are interested in removing any compactness condition. For these reasons we focus on the equation 


$$
\begin{cases}-\epsilon^{2} \Delta u+\lambda u+\rho(x) \phi u=|u|^{p-1} u, & x \in \mathbb{R}^{3} \\ -\Delta \phi=\rho(x) u^{2}, & x \in \mathbb{R}^{3}\end{cases}
$$

with $\rho: \mathbb{R}^{3} \rightarrow \mathbb{R}$ a nonnegative measurable function, $\lambda \in \mathbb{R}$, and $\lambda>0$, taking advantage of a shrinking parameter $\epsilon \sim \hbar \ll 1$ which behaves like the Planck constant in the so-called 'semiclassical limit'. In this direction, Ianni and Vaira [29] notably showed that concentration of semiclassical solutions to

$$
\begin{cases}-\epsilon^{2} \Delta u+V(x) u+\rho(x) \phi u=|u|^{p-1} u, & 1<p<5, \quad x \in \mathbb{R}^{3} \\ -\Delta \phi=\rho(x) u^{2}, & x \in \mathbb{R}^{3},\end{cases}
$$

occurs at stationary points of the external potential $V$ using a Lyapunov-Schmidt approach (in the spirit of the Ambrosetti-Malchiodi monograph [3] on perturbation methods), whereas in [14] concentration results have been obtained using a variational/penalisation approach in the spirit of del Pino and Felmer [24]. In particular, in [14] the question of studying concentration phenomena which are purely driven by $\rho$ has been raised. None of the aforementioned contributions have dealt with necessary conditions for concentration at points in the case $V \equiv$ constant and in the presence of a variable charge density function $\rho$. We manage to fill this gap, by obtaining a necessary condition, related to $\rho$, for the concentration at points for solutions to (1.15) both in $H^{1}\left(\mathbb{R}^{3}\right)$ and in $E\left(\mathbb{R}^{3}\right)$, which are the suitable settings for the study of concentration phenomena with perturbative and variational techniques, respectively.

Theorem 5. [Necessary conditions in E] Suppose that $\rho \in C^{1}\left(\mathbb{R}^{3}\right)$ is nonnegative and $|\nabla \rho(x)|=O\left(|x|^{a} e^{b|x|}\right)$ as $|x| \rightarrow+\infty$ for some $a>0$ and some $b \in \mathbb{R}$. Let $p \in[2,5)$ and let $\left(u_{\epsilon}, \phi_{u_{\epsilon}}\right) \in E\left(\mathbb{R}^{3}\right) \times D^{1,2}\left(\mathbb{R}^{3}\right)$ be a sequence of positive solutions of (1.15). Assume that $u_{\epsilon}$ concentrate at a point $x_{0}$ for sufficiently small $\epsilon$, meaning that $\forall \delta>0, \exists \epsilon_{0}>0, \exists R>0$ such that $u_{\epsilon}(x) \leq \delta$ for $\left|x-x_{0}\right| \geq \epsilon R, \epsilon<\epsilon_{0}$. Then, $\nabla \rho\left(x_{0}\right)=0$.

The above result is obtained in the spirit of [51] using classical blow-up analysis, uniform decay estimates, and Pohozaev type identities.

Remark 5. Since we deal with concentrating solutions, we use the mean value theorem to control the growth of $\rho$ with the assumption on $\nabla \rho$ in order to apply the dominated convergence theorem in the proof of the theorem (see Claim 5). We note that this assumption is not needed in Theorem 6 as we work with a bounded $\rho$ and therefore the application of the dominated convergence theorem is more immediate.

Remark 6. When $b>0$ the proof of Theorem 5 Claim 5 is sensitive to $\epsilon$ being smaller than the ratio $\frac{\sqrt{\lambda}}{b}$. This ratio arises as the proof consists of balancing the aforementioned growth of $\rho$ and $\nabla \rho$ with the a priori exponential decay of the concentrating solutions in order to apply the dominated convergence theorem.

Theorem 6. [Necessary conditions in $\left.H^{1}\right]$ Suppose that $\rho \in C^{1}\left(\mathbb{R}^{3}\right)$ is nonnegative and that $\rho, \nabla \rho$ are bounded. Let $p \in[2,5)$ and let $\left(u_{\epsilon}, \phi_{u_{\epsilon}}\right) \in H^{1}\left(\mathbb{R}^{3}\right) \times D^{1,2}\left(\mathbb{R}^{3}\right)$ be a sequence of positive solutions of (1.15). Assume that $u_{\epsilon}$ concentrate at a point $x_{0}$, meaning that $\forall \delta>0, \exists \epsilon_{0}>0$, $\exists R>0$ such that $u_{\epsilon}(x) \leq \delta$ for $\left|x-x_{0}\right| \geq \epsilon R, \epsilon<\epsilon_{0}$. Then, $\nabla \rho\left(x_{0}\right)=0$. 
Remark 7. It is possible to relax the global boundedness assumption on $\rho$ and/or on $\nabla \rho$ when working in $H^{1}\left(\mathbb{R}^{3}\right)$, if we make a growth assumption on $\nabla \rho$. Namely, if we work in $H^{1}\left(\mathbb{R}^{3}\right)$ and have adequate local integrability on $\rho$ to ensure $\int_{\mathbb{R}^{3}} \int_{\mathbb{R}^{3}} \frac{u^{2}(x) \rho(x) u^{2}(y) \rho(y)}{|x-y|} \mathrm{d} x \mathrm{~d} y<+\infty$, typically identified using the Hardy-Littlewood-Sobolev inequality, the statement of the theorem and the proof is identical to that of Theorem 5 .

Remark 8. In the proof of both Theorem 5 and Theorem 6 , one actually finds the condition $\rho\left(x_{0}\right) \nabla \rho\left(x_{0}\right)=0$. We believe that this may be a necessary condition in the case $\rho$ is allowed to change sign on a small region.

1.2. Related questions. In our opinion, there are a number of interesting questions related to our work which are worth studying in future projects.

A. Radial versus non-radial solutions. In the case $\rho$ is a radial function one can restrict on functions having the same symmetry to find radial solutions, using Palais criticality principle, in all the above scenarios (coercive/non-coercive cases, for low/large $p$ ). It is not clear how to compare the energy levels nor the symmetry of the solutions with those that one finds using the above non-radial approaches.

B. Variational characterisation. As mentioned above, when $p \in(2,3]$, it is not obvious whether the mountain pass critical points for $I$, are least energy solutions. Namely, for $p \in(2,3]$, there is no clear relation between the solutions found in Theorem 1 (for $p=3$ ) and Theorem 2 with those found in Corollary 1, as well as between the solutions found in Theorem 4 (for $p=3$ ) and Theorem 3 with those found in Corollary 2.

C. Multiplicity. For $p>3$, we believe that the existence of infinitely many solutions can be proved following Ambrosetti-Ruiz paper [5], for instance in the case of (possibly non-radial) coercive $\rho$. We suspect that the constrained minimisation approach in [49] may help refining the approach in [5], to obtain a multiplicity result for $p \leq 3$.

D. 'Sharp' necessary conditions for point concentration. Is it possible to allow a faster growth for $\rho$ in the necessary conditions for point concentration? The proof we provide is based on the uniform exponential decay of solutions, which is essentially due to the $L^{2}$ setting.

E. Sufficient conditions for point concentration. Following a personal communication of Denis Bonheure [13] setting

$$
I_{\epsilon}(u)=\frac{1}{2} \int_{\mathbb{R}^{3}}\left(\epsilon^{2}|\nabla u|^{2}+u^{2}\right)+\frac{1}{4} \int_{\mathbb{R}^{3}} \rho \phi_{u} u^{2}-\frac{1}{p+1} \int_{\mathbb{R}^{3}}|u|^{p+1}
$$

and

$$
I_{0}(u)=\frac{1}{2} \int_{\mathbb{R}^{3}}\left(|\nabla u|^{2}+u^{2}\right)-\frac{1}{p+1} \int_{\mathbb{R}^{3}}|u|^{p+1},
$$

taking $u \in C_{c}^{1}\left(\mathbb{R}^{3}\right)$ and using the scaling

$$
u_{\epsilon}=u\left(\frac{\cdot-x_{0}}{\epsilon}\right),
$$

one finds the expansion

$$
\frac{\epsilon^{-3} I_{\epsilon}\left(u_{\epsilon}\right)-I_{0}(u)}{\epsilon^{2}} \approx \rho^{2}\left(x_{0}\right) \int_{\mathbb{R}^{3}} \int_{\mathbb{R}^{3}} \frac{u^{2}(y) u^{2}(x)}{4 \pi|x-y|} \mathrm{d} y \mathrm{~d} x, \quad \epsilon \ll 1 .
$$


Inspired by this formal calculation, in a forthcoming paper, we will prove concentration at strict local minima of $\rho^{2}$.

1.3. Outline. The paper is organised as follows. In Section 2, we recall some properties of the space $E\left(\mathbb{R}^{3}\right)$, prove regularity and positivity results for solutions to the nonlinear SchrödingerPoisson system, and establish a useful Pohozaev identity for these solutions, the proof of which can be found in the appendix. In Section 3, we outline the min-max setting and define the levels $c_{\mu}$ and $c_{\mu}^{\infty}, c$, and $c^{\infty}$, which are relevant for both the coercive and non-coercive cases. We then find lower bounds on the functions $I_{\mu}$ and $I_{\mu}^{\infty}$, when restricted to the set of nontrivial solutions which are fundamental in relation to compactness properties of Palais-Smale sequences. In Section 4, we study the case of a coercive $\rho$ and establish that this coercivity is a sufficient condition for the compactness of the embedding $E\left(\mathbb{R}^{3}\right) \hookrightarrow L^{p+1}\left(\mathbb{R}^{3}\right)$. This enables us, using the min-max setting of Section 3, to prove existence of mountain pass solutions in the coercive case for $p \in(2,3)$ (Theorem 1 ). We then use a minimisation argument to prove the existence of least energy solutions (Corollary 1). In Section 5, we focus on a non-coercive $\rho$ and we first establish a representation result for bounded Palais-Smale sequence for $I_{\mu}$ in Proposition 2. Using the min-max setting of Section 3 and the lower bounds found in this section, we prove existence of mountain pass solutions for $p \in(2,3)$ (Theorem 3 ). We then show that for $p \geq 3$ the PalaisSmale condition holds for $I$ at the level $c$, following which the proof of Theorem 4 easily follows. We finally prove the existence of least energy solutions in the non-coercive case for $p \in(2,5)$ (Corollary 2). In Section 6, we obtain necessary conditions for the concentration at points in both $E$ (Theorem 5) and $H^{1}$ (Theorem 6).

1.4. Notation. We use the following notation throughout:

- $L^{p}(\Omega)$, with $\Omega \subseteq \mathbb{R}^{3}$ and $p \geq 1$, is the usual Lebesgue space. $L^{p}\left(\mathbb{R}^{3}\right)=L^{p}$.

- The Hölder space $C^{k, \alpha}(\Omega)$, with $\Omega \subseteq \mathbb{R}^{3}$ and $\alpha \in(0,1]$, is the set of functions on $\Omega$ that are $k$-fold differentiable and whose $k$-fold derivatives are Hölder continuous of order $\alpha$.

- $H^{1}, W^{m, p}$ are classical Sobolev spaces.

- $H^{-1}\left(\mathbb{R}^{3}\right)=H^{-1}$ denotes the dual space of $H^{1}\left(\mathbb{R}^{3}\right)$.

- $D^{1,2}\left(\mathbb{R}^{3}\right)=D^{1,2}$ is the space defined as

$$
D^{1,2}\left(\mathbb{R}^{3}\right):=\left\{u \in L^{6}\left(\mathbb{R}^{3}\right): \nabla u \in L^{2}\left(\mathbb{R}^{3}\right)\right\},
$$

and equipped with norm

$$
\|u\|_{D^{1,2}\left(\mathbb{R}^{3}\right)}:=\|\nabla u\|_{L^{2}\left(\mathbb{R}^{3}\right)} .
$$

- $E\left(\mathbb{R}^{3}\right)=E$ is the space defined as

$$
E\left(\mathbb{R}^{3}\right):=\left\{u \in D^{1,2}\left(\mathbb{R}^{3}\right):\|u\|_{E}<+\infty\right\},
$$

where

$$
\|u\|_{E}^{2}:=\int_{\mathbb{R}^{3}}\left(|\nabla u|^{2}+u^{2}\right) \mathrm{d} x+\left(\int_{\mathbb{R}^{3}} \int_{\mathbb{R}^{3}} \frac{u^{2}(x) \rho(x) u^{2}(y) \rho(y)}{|x-y|} \mathrm{d} x \mathrm{~d} y\right)^{1 / 2} .
$$

- We set

$$
\phi_{u}(x):=\int_{\mathbb{R}^{3}} \frac{\rho(y) u^{2}(y)}{4 \pi|x-y|} \mathrm{d} y
$$

and

$$
\bar{\phi}_{u}(x):=\int_{\mathbb{R}^{3}} \frac{\rho_{\infty} u^{2}(y)}{4 \pi|x-y|} \mathrm{d} y .
$$


- For any $\eta>0$ and any $z \in \mathbb{R}^{3}, B_{\eta}(z)$ is the ball of radius $\eta$ centered at $z$. For any $\eta>0$, $B_{\eta}$ is the ball of radius $\eta$ centered at 0 .

- $S_{p+1}:=\inf _{u \in H^{1}\left(\mathbb{R}^{3}\right) \backslash\{0\}} \frac{\|u\|_{H^{1}\left(\mathbb{R}^{3}\right)}^{2}}{\|u\|_{L^{p+1}\left(\mathbb{R}^{3}\right)}^{2}}$ is the best Sobolev constant for the embedding of $H^{1}\left(\mathbb{R}^{3}\right)$ into $L^{p+1}\left(\mathbb{R}^{3}\right)$.

- Let $A \subset \mathbb{R}^{3}$. Then, we define

$$
\chi_{A}(x):= \begin{cases}1, & x \in A, \\ 0, & x \notin A .\end{cases}
$$

- $C, C_{1}, C^{\prime}$, etc., denote generic positive constants.

- Asymptotic Notation: For real valued functions $f(t), g(t) \geq 0$, we write:

$-f(t) \lesssim g(t)$ if there exists $C>0$ independent of $t$ such that $f(t) \leq C g(t)$.

$-f(t)=o(g(t))$ as $t \rightarrow+\infty$ if and only if $g(t) \neq 0$ and $\lim _{t \rightarrow+\infty} \frac{f(t)}{g(t)}=0$.

- $f(t)=O(g(t))$ as $t \rightarrow+\infty$ if and only if there exists $C_{1}>0$ such that $f(t) \leq C_{1} g(t)$ for $t$ large.

\section{ACKNowledgements}

C.M. would like to thank Antonio Ambrosetti and David Ruiz for having drawn his attention to questions related to the Schrödinger-Poisson systems. The same author would like to thank Michel Willem for inspiring discussions on questions related to the Palais-Smale condition. Both authors would like to thank Norihisa Ikoma for his suggestion to consider a minimising sequence in order to prove the existence of least energy solutions. Last, but not least, both authors would like to thank an anonymous referee for the valuable and constructive comments.

\section{Preliminaries}

2.1. The space $E\left(\mathbb{R}^{3}\right)$. Let us assume that $\rho$ is continuous and nonnegative. It is easy to see that $E\left(\mathbb{R}^{3}\right)$ is a uniformly convex Banach space. As a consequence it is reflexive and, in particular, the unit ball is weakly compact. Reasoning as in Proposition 2.4 in [44] and Proposition 2.10 in [40] a sequence $\left(u_{n}\right)_{n \in \mathbb{N}} \subset E\left(\mathbb{R}^{3}\right)$ is weakly convergent to $u \in E$ if and only if is bounded and converges in $L_{\text {loc }}^{1}\left(\mathbb{R}^{3}\right)$. In particular, $\phi_{u_{n}} \rightarrow \phi_{u}$ in $D^{1,2}\left(\mathbb{R}^{3}\right)$. The following nonlocal Brezis-Lieb lemma is very useful to study the compactness of Palais-Smale sequences.

Lemma 2.1 ([7], [40]). [Nonlocal Brezis-Lieb lemma] Let $\left(u_{n}\right)_{n \in \mathbb{N}} \subset E\left(\mathbb{R}^{3}\right)$ be a bounded sequence such that $u_{n} \rightarrow u$ almost everywhere in $\mathbb{R}^{3}$. Then it holds that

$$
\lim _{n \rightarrow \infty}\left[\left\|\nabla \phi_{u_{n}}\right\|_{L^{2}\left(\mathbb{R}^{3}\right)}^{2}-\left\|\nabla \phi_{u_{n}-u}\right\|_{L^{2}\left(\mathbb{R}^{3}\right)}^{2}\right]=\left\|\nabla \phi_{u}\right\|_{L^{2}\left(\mathbb{R}^{3}\right)}^{2} .
$$

\subsection{Regularity and positivity.}

Proposition 3. [Regularity and positivity] Let $p \in[1,5], \rho \in L_{\text {loc }}^{\infty}\left(\mathbb{R}^{3}\right) \backslash\{0\}$ be nonnegative and $\left(u, \phi_{u}\right) \in E\left(\mathbb{R}^{3}\right) \times D^{1,2}\left(\mathbb{R}^{3}\right)$ be a weak solution of the problem

$$
\begin{cases}-\Delta u+b u+c \rho(x) \phi u=d|u|^{p-1} u, & x \in \mathbb{R}^{3} \\ -\Delta \phi=\rho(x) u^{2}, & x \in \mathbb{R}^{3}\end{cases}
$$

with $b, c, d \in \mathbb{R}_{+}$. Assume that $u$ is nonnegative. Then, $u, \phi_{u} \in W_{l o c}^{2, q}\left(\mathbb{R}^{3}\right)$, for every $q \geq 1$, and so $u, \phi_{u} \in C_{\text {loc }}^{1, \alpha}\left(\mathbb{R}^{3}\right)$. If, in addition, $u \neq \equiv$, then $u, \phi_{u}>0$ everywhere. 
Proof. Under the hypotheses of the proposition, both $u$ and $\phi_{u}$ have weak second derivatives in $L_{\text {loc }}^{q}$ for all $q<\infty$. In fact, note that from the first equation in $(2.1)$, we have that $-\Delta u=g(x, u)$, where

$$
\begin{aligned}
|g(x, u)| & =\mid\left(-b u-c \rho(x) \phi u+d|u|^{p-1} u \mid\right. \\
& \leq C\left(1+\left|\rho \phi_{u}\right|+|u|^{p-1}\right)(1+|u|) \\
& :=h(x)(1+|u|) .
\end{aligned}
$$

Using our assumptions on $\rho, \phi_{u}$, and $u$, we can show that $h \in L_{\text {loc }}^{3 / 2}\left(\mathbb{R}^{3}\right)$, which implies that $u \in L_{\text {loc }}^{q}\left(\mathbb{R}^{3}\right)$ for all $q<+\infty$ (see e.g. [48, p. 270]). Note that since $u^{2} \rho \in L_{\text {loc }}^{q}\left(\mathbb{R}^{3}\right)$ for all $q<+\infty$, then by the second equation in (2.1) and the Calderón-Zygmund estimates, we have that $\phi_{u} \in W_{\text {loc }}^{2, q}\left(\mathbb{R}^{3}\right)$ (see e.g. [27]). This then enables us to show that $g \in L_{\text {loc }}^{q}\left(\mathbb{R}^{3}\right)$ for all $q<+\infty$, which implies, by Calderón-Zygmund estimates, that $u \in W_{\text {loc }}^{2, q}\left(\mathbb{R}^{3}\right)$ (see e.g. [27]). The $C_{\text {loc }}^{1, \alpha}\left(\mathbb{R}^{3}\right)$ regularity of both $u, \phi_{u}$ is a consequence of Morrey's embedding theorem. Finally, the strict positivity is a consequence of the strong maximum principle, and this concludes the proof.

Remark 9. If, in addition, $\rho \in C_{\text {loc }}^{0, \alpha}\left(\mathbb{R}^{3}\right)$, then, by Schauder's estimates on both equations, it holds that $u, \phi_{u} \in C_{\text {loc }}^{2, \alpha}\left(\mathbb{R}^{N}\right)$.

2.3. Pohozaev identity. We can now establish a useful Pohozaev type identity for solutions to the nonlinear Schrödinger-Poisson system that will be used on numerous occasions. Although these kind of identities are standard, since we do not find a precise reference, we give a proof in the appendix for the reader convenience.

Lemma 2.2. [Pohozaev identity] Assume $\rho \in L_{\text {loc }}^{\infty}\left(\mathbb{R}^{3}\right) \cap W_{\text {loc }}^{1,1}\left(\mathbb{R}^{3}\right)$ is nonnegative and $p \in[1,5]$. Let $\left(u, \phi_{u}\right) \in E\left(\mathbb{R}^{3}\right) \times D^{1,2}\left(\mathbb{R}^{3}\right)$ be a weak solution of the problem (2.1). Then, it holds that

$$
\left|\frac{c}{2} \int_{\mathbb{R}^{3}} \phi_{u} u^{2}(x, \nabla \rho) \mathrm{d} x\right|<+\infty{ }^{1}
$$

and

$$
\frac{1}{2} \int_{\mathbb{R}^{3}}|\nabla u|^{2}+\frac{3 b}{2} \int_{\mathbb{R}^{3}} u^{2}+\frac{5 c}{4} \int_{\mathbb{R}^{3}} \rho \phi_{u} u^{2}+\frac{c}{2} \int_{\mathbb{R}^{3}} \phi_{u} u^{2}(x, \nabla \rho)-\frac{3 d}{p+1} \int_{\mathbb{R}^{3}}|u|^{p+1}=0 .
$$

\section{The min-maX Setting: Definition of $c_{\mu}, c_{\mu}^{\infty}, c$, AND $c^{\infty}$}

In what is to come, we will first examine the existence of solutions of (1.1) in the case of a coercive potential $\rho$ (see Section 4). The appropriate setting for this problem will be the space $E\left(\mathbb{R}^{3}\right) \subset H^{1}\left(\mathbb{R}^{3}\right)$. We begin by recalling that solving (1.1) reduces to solving (1.9) with

\footnotetext{
${ }^{1}$ In the case $(x, \nabla \rho)$ changes sign, we set

$$
\int_{\mathbb{R}^{3}} \phi_{u} u^{2}(x, \nabla \rho) \mathrm{d} x=\lim _{n \rightarrow \infty} \int_{B_{R_{n}}} \phi_{u} u^{2}(x, \nabla \rho) \mathrm{d} x
$$

for a suitable sequence of radii $R_{n} \rightarrow \infty$. As part of the proof we can select a sequence $\left(R_{n}\right)_{n \in \mathbb{N}}$ such that this limit exists and is finite.
} 
$\phi_{u}(x):=\int_{\mathbb{R}^{3}} \frac{u^{2}(y) \rho(y)}{\omega|x-y|} d y \in D^{1,2}\left(\mathbb{R}^{3}\right)$. It will also be useful to introduce a perturbation of (1.9), namely

$$
-\Delta u+u+\rho(x) \phi_{u} u=\mu|u|^{p-1} u, \quad \mu \in\left[\frac{1}{2}, 1\right]
$$

and to note that the positive solutions of this perturbed problem are critical points of the corresponding functional $I_{\mu}: E\left(\mathbb{R}^{3}\right) \rightarrow \mathbb{R}$, defined as

$$
I_{\mu}(u):=\frac{1}{2} \int_{\mathbb{R}^{3}}\left(|\nabla u|^{2}+u^{2}\right)+\frac{1}{4} \int_{\mathbb{R}^{3}} \rho \phi_{u} u^{2}-\frac{\mu}{p+1} \int_{\mathbb{R}^{3}} u_{+}^{p+1}, \quad \mu \in\left[\frac{1}{2}, 1\right] .
$$

We will now show that $I_{\mu}$ has the mountain pass geometry in $E$ for each $\mu \in\left[\frac{1}{2}, 1\right]$.

Lemma 3.1. [Mountain-Pass Geometry for $I_{\mu}$ ] Suppose $\rho \in C\left(\mathbb{R}^{3}\right)$ is nonnegative and $p \in$ $(2,5]$. Then, for each $\mu \in\left[\frac{1}{2}, 1\right]$, it holds:

(i) $I_{\mu}(0)=0$ and there exists constants $r, a>0$ such that $I_{\mu}(u) \geq a$ if $\|u\|_{E}=r$.

(ii) There exists $v \in E$ with $\|v\|_{E}>r$, such that $I_{\mu}(v) \leq 0$.

Proof. We follow Lemma 14 in [15]. To prove $(i)$ note that since $H^{1}\left(\mathbb{R}^{3}\right) \hookrightarrow L^{p+1}\left(\mathbb{R}^{3}\right)$ then for some constant $C>0$, it holds that

$$
I_{\mu}(u) \geq \frac{1}{2}\|u\|_{H^{1}}^{2}+\frac{1}{4} \int_{\mathbb{R}^{3}} \rho \phi_{u} u^{2}-C \mu\|u\|_{H^{1}}^{p+1}
$$

Now, from the definition of the norm in $E$ we can see that $4 \pi \int_{\mathbb{R}^{3}} \rho \phi_{u} u^{2}=\left(\|u\|_{E}^{2}-\|u\|_{H^{1}}^{2}\right)^{2}$. Therefore, we have that

$$
\begin{aligned}
I_{\mu}(u) & \geq \frac{1}{2}\|u\|_{H^{1}}^{2}+\frac{1}{16 \pi}\left(\|u\|_{E}^{2}-\|u\|_{H^{1}}^{2}\right)^{2}-C \mu\|u\|_{H^{1}}^{p+1} \\
& =\frac{1}{2}\|u\|_{H^{1}}^{2}+\frac{1}{4 \pi}\left(\frac{1}{4}\|u\|_{E}^{4}-\frac{1}{2}\|u\|_{E}^{2}\|u\|_{H^{1}}^{2}+\frac{1}{4}\|u\|_{H^{1}}^{4}\right)-C \mu\|u\|_{H^{1}}^{p+1} .
\end{aligned}
$$

For some $\alpha \neq 0$, using the elementary inequality

$$
\frac{1}{2}\|u\|_{E}^{2}\|u\|_{H^{1}}^{2} \leq \frac{\alpha^{2}}{4}\|u\|_{H^{1}}^{4}+\frac{1}{4 \alpha^{2}}\|u\|_{E}^{4}
$$

we have

$$
\begin{aligned}
I_{\mu}(u) & \geq \frac{1}{2}\|u\|_{H^{1}}^{2}+\frac{1}{4 \pi}\left(\frac{1}{4}\|u\|_{E}^{4}-\frac{\alpha^{2}}{4}\|u\|_{H^{1}}^{4}-\frac{1}{4 \alpha^{2}}\|u\|_{E}^{4}+\frac{1}{4}\|u\|_{H^{1}}^{4}\right)-C \mu\|u\|_{H^{1}}^{p+1} \\
& =\frac{1}{2}\|u\|_{H^{1}}^{2}-\frac{1}{4 \pi}\left(\frac{\alpha^{2}-1}{4}\right)\|u\|_{H^{1}}^{4}+\frac{1}{4 \pi}\left(\frac{\alpha^{2}-1}{4 \alpha^{2}}\right)\|u\|_{E}^{4}-C \mu\|u\|_{H^{1}}^{p+1} .
\end{aligned}
$$


We now assume $\|u\|_{E}<\delta$ for some $\delta>0$, which also implies that $\|u\|_{H^{1}}^{2}<\delta^{2}$, and we take $\alpha>1$. Then, from (3.3), we see that

$$
\begin{aligned}
I_{\mu}(u) & \geq\left[\frac{1}{2}-\frac{1}{4 \pi}\left(\frac{\alpha^{2}-1}{4}\right) \delta^{2}-C \mu \delta^{p-1}\right]\|u\|_{H^{1}}^{2}+\frac{1}{4 \pi}\left(\frac{\alpha^{2}-1}{4 \alpha^{2}}\right)\|u\|_{E}^{4} \\
& \geq \frac{1}{4 \pi}\left(\frac{\alpha^{2}-1}{4 \alpha^{2}}\right)\|u\|_{E}^{4}, \quad \text { for } \delta \text { sufficiently small. }
\end{aligned}
$$

Hence, we have shown that the origin is a strict local minimum for $I_{\mu}$ in $E$ if $p \in[2,5]$.

To show $(i i)$, pick $u \in C^{1}\left(\mathbb{R}^{3}\right)$, supported in the unit ball, $B_{1}$. Setting $v_{t}(x):=t^{2} u(t x)$ we find that

$$
I_{\mu}\left(v_{t}\right)=\frac{t^{3}}{2} \int_{\mathbb{R}^{3}}|\nabla u|^{2}+\frac{t}{2} \int_{\mathbb{R}^{3}} u^{2}+\frac{t^{3}}{4} \int_{\mathbb{R}^{3}} \int_{\mathbb{R}^{3}} \frac{u^{2}(y) \rho\left(\frac{y}{t}\right) u^{2}(x) \rho\left(\frac{x}{t}\right)}{\omega|x-y|} \mathrm{d} y \mathrm{~d} x-\frac{\mu t^{2 p-1}}{p+1} \int_{\mathbb{R}^{3}} u_{+}^{p+1}
$$

Since the Poisson term is uniformly bounded, namely for $t>1$

$$
\int_{\mathbb{R}^{3}} \int_{\mathbb{R}^{3}} \frac{u^{2}(y) \rho\left(\frac{y}{t}\right) u^{2}(x) \rho\left(\frac{x}{t}\right)}{\omega|x-y|} \mathrm{d} y \mathrm{~d} x \leq\|\rho\|_{L^{\infty}\left(B_{1}\right)}^{2} \int_{\mathbb{R}^{3}} \int_{\mathbb{R}^{3}} \frac{u^{2}(y) u^{2}(x)}{\omega|x-y|} \mathrm{d} y \mathrm{~d} x<+\infty,
$$

the fact that $2 p-1>3$ in (3.4) yields $I_{\mu}\left(v_{t}\right) \rightarrow-\infty$ as $t \rightarrow+\infty$, and this is enough to prove (ii). This concludes the proof.

The previous lemma, as well as the monotonicity of $I_{\mu}$ with respect to $\mu$, imply that there exists $\bar{v} \in E \backslash\{0\}$ such that

$$
I_{\mu}(\bar{v}) \leq I_{\frac{1}{2}}(\bar{v}) \leq 0, \quad \forall \mu \in\left[\frac{1}{2}, 1\right]
$$

Thus, we can define, in the spirit of Ambrosetti-Rabinowitz [4], the min-max level associated with $I_{\mu}$ as

$$
c_{\mu}:=\inf _{\gamma \in \Gamma} \max _{t \in[0,1]} I_{\mu}(\gamma(t))
$$

where $\Gamma$ is the family of paths

$$
\Gamma:=\{\gamma \in C([0,1], E): \gamma(0)=0, \gamma(1)=\bar{v}\}
$$

It is worth emphasising that to apply the monotonicity trick [30] and [31] it is essential that the above class $\Gamma$ does not depend on $\mu$.

Lemma 3.2. Suppose $\rho \in C\left(\mathbb{R}^{3}\right)$ is nonnegative and $p \in(2,5)$. Then:

(i) The mapping $\left[\frac{1}{2}, 1\right] \ni \mu \mapsto c_{\mu}$ is non-increasing and left-continuous. 
(ii) For almost every $\mu \in\left[\frac{1}{2}, 1\right]$, there exists a bounded Palais-Smale sequence for $I_{\mu}$ at the level $c_{\mu}$. That is, there exists a bounded sequence $\left(u_{n}\right)_{n \in \mathbb{N}} \subset E$ such that $I_{\mu}\left(u_{n}\right) \rightarrow c_{\mu}$ and $I_{\mu}^{\prime}\left(u_{n}\right) \rightarrow 0$.

Proof. The proof of $(i)$ follows from Lemma 2.2 in [5]. To prove (ii), we notice that by Lemma 3.1 , it holds that

$$
c_{\mu}=\inf _{\gamma \in \Gamma} \max _{t \in[0,1]} I_{\mu}(\gamma(t))>0 \geq \max \left\{I_{\mu}(0), I_{\mu}(\bar{v})\right\}, \quad \forall \mu \in\left[\frac{1}{2}, 1\right] .
$$

Thus, the result follows by Theorem 1.1 in [30].

With the previous result in place, we can define the set

$$
\mathcal{M}:=\left\{\mu \in\left[\frac{1}{2}, 1\right]: \exists \text { bounded Palais-Smale sequence for } I_{\mu} \text { at the level } c_{\mu}\right\}
$$

Since $I$ has the mountain pass geometry by Lemma 3.1, using $(i)$ of Lemma 3.2, we can now define the min-max level associated with $I$ as

$$
c:= \begin{cases}c_{1}, & p \in(2,3), \\ \inf _{\gamma \in \bar{\Gamma}} \max _{t \in[0,1]} I(\gamma(t)), & p \in[3,5),\end{cases}
$$

where $\bar{\Gamma}$ is the family of paths

$$
\bar{\Gamma}:=\left\{\gamma \in C\left([0,1], E\left(\mathbb{R}^{3}\right)\right): \gamma(0)=0, I(\gamma(1))<0\right\}
$$

This finalises the preliminary min-max scheme for the case of a coercive $\rho$.

In Section 5, we will then focus on the case of non-coercive $\rho$, namely $\rho(x) \rightarrow \rho_{\infty}$ as $|x| \rightarrow+\infty$, and the appropriate setting for this problem will be the space $H^{1}\left(\mathbb{R}^{3}\right)$. It will once again be useful to introduce a perturbation of (1.9), namely, (3.1), and to recall that the positive solutions of this perturbed problem are critical points of the corresponding functional, $I_{\mu}: H^{1}\left(\mathbb{R}^{3}\right) \rightarrow \mathbb{R}$, defined in (3.2). We note that Lemma 3.1 and Lemma 3.2 hold with $E\left(\mathbb{R}^{3}\right)=H^{1}\left(\mathbb{R}^{3}\right)$, and thus $\mathcal{M}$ can be defined as in (3.6). We now introduce the problem at infinity related to (3.1) in this case, namely

$$
-\Delta u+u+\rho_{\infty} \bar{\phi}_{u} u=\mu|u|^{p-1} u, \quad \mu \in\left[\frac{1}{2}, 1\right],
$$

where $\bar{\phi}_{u}(x):=\int_{\mathbb{R}^{3}} \frac{\rho_{\infty} u^{2}(y)}{\omega|x-y|} d y \in D^{1,2}\left(\mathbb{R}^{3}\right)$. Positive solutions of (3.8) are critical points of the corresponding functional, $I_{\mu}^{\infty}: H^{1}\left(\mathbb{R}^{3}\right) \rightarrow \mathbb{R}$, defined as

$$
I_{\mu}^{\infty}(u):=\frac{1}{2} \int_{\mathbb{R}^{3}}\left(|\nabla u|^{2}+u^{2}\right)+\frac{1}{4} \int_{\mathbb{R}^{3}} \rho_{\infty} \bar{\phi}_{u} u^{2}-\frac{\mu}{p+1} \int_{\mathbb{R}^{3}} u_{+}^{p+1}, \quad \mu \in\left[\frac{1}{2}, 1\right] .
$$

It can be shown that $I_{\mu}^{\infty}$ satisfies the geometric conditions of the mountain-pass theorem, using similar arguments as those used in the proof of Lemma 3.1. We therefore define the min-max level associated with $I_{\mu}^{\infty}$ as 


$$
c_{\mu}^{\infty}:=\inf _{\gamma \in \Gamma^{\infty}} \max _{t \in[0,1]} I_{\mu}^{\infty}(\gamma(t))
$$

where

$$
\Gamma^{\infty}:=\left\{\gamma \in C\left([0,1], H^{1}\left(\mathbb{R}^{3}\right)\right): \gamma(0)=0, I_{\frac{1}{2}}^{\infty}(\gamma(1))<0\right\}
$$

Moreover, we define the min-max level associated with $I^{\infty}$ as

$$
c^{\infty}:= \begin{cases}c_{1}^{\infty}, & p \in(2,3), \\ \inf _{\gamma \in \bar{\Gamma}^{\infty}} \max _{t \in[0,1]} I^{\infty}(\gamma(t)), & p \in[3,5),\end{cases}
$$

where $\bar{\Gamma}^{\infty}$ is the family of paths

$$
\bar{\Gamma}^{\infty}:=\left\{\gamma \in C\left([0,1], E\left(\mathbb{R}^{3}\right)\right): \gamma(0)=0, I^{\infty}(\gamma(1))<0\right\} .
$$

3.1. Lower bounds for $I$ and $I^{\infty}$. In the next two lemmas, we establish lower bounds on $I_{\mu}$ and $I_{\mu}^{\infty}$, when restricted to nonnegative and nontrivial solutions of (3.1) and (3.8), respectively. These bounds will be used on numerous occasions.

Lemma 3.3. Suppose $\rho \in C\left(\mathbb{R}^{3}\right)$ is nonnegative and $\mu \in\left[\frac{1}{2}, 1\right]$. Define $\mathcal{A}:=\left\{u \in H^{1}\left(\mathbb{R}^{3}\right) \backslash\{0\}\right.$ : $u$ is a nonnegative solution to (3.1) $\}$. Then, if $p \in[3,5)$, it holds that

$$
\inf _{u \in \mathcal{A}} I_{\mu}(u) \geq \frac{p-1}{2(p+1)}\left(S_{p+1}\right)^{\frac{p+1}{p-1}}>0 .
$$

If $p \in(2,3)$, suppose, in addition, $\rho \in W_{l o c}^{1,1}\left(\mathbb{R}^{3}\right)$ and $k \rho(x) \leq(x, \nabla \rho)$ for some $k>\frac{-2(p-2)}{(p-1)}$. Then, it holds that

$$
\inf _{u \in \mathcal{A}} I_{\mu}(u) \geq C(k, p)
$$

with

$$
C(k, p):=\left(\frac{2(p-2)+k(p-1)}{(3+2 k)(p+1)}\right)\left(S_{p+1}\right)^{\frac{p+1}{p-1}}>0
$$

Proof. Let $\bar{u} \in H^{1}\left(\mathbb{R}^{3}\right) \backslash\{0\}$ be an arbitrary nonnegative solution of $(3.1)$ such that $I_{\mu}(\bar{u})=\bar{c}$. Using the Sobolev embedding theorem and the fact that $I_{\mu}^{\prime}(\bar{u})(\bar{u})=0$, we see that

$$
S_{p+1}\|\bar{u}\|_{L^{p+1}}^{2} \leq\|\bar{u}\|_{H^{1}}^{2} \leq\|\bar{u}\|_{H^{1}}^{2}+\int_{\mathbb{R}^{3}} \rho \phi_{\bar{u}} \bar{u}^{2}=\mu\|\bar{u}\|_{L^{p+1}}^{p+1} .
$$

Since $\mu \leq 1$ it follows that

$$
\left(S_{p+1}\right)^{\frac{p+1}{p-1}} \leq\|\bar{u}\|_{H^{1}}^{2}
$$

If $p \in[3,5)$, using the definition of $\bar{c}$ and Nehari's condition, we can see that 


$$
\left(\frac{1}{2}-\frac{1}{p+1}\right)\|\bar{u}\|_{H^{1}}^{2} \leq \bar{c}
$$

and so the bound on $\bar{c}$ immediately follows from (3.12). If $p \in(2,3)$, we first note that since $I_{\mu}(\bar{u})=\bar{c}, I_{\mu}^{\prime}(\bar{u})(\bar{u})=0$, and $\bar{u}=(\bar{u})_{+}$, then $\bar{u}$ satisfies

$$
\frac{1}{2} \int_{\mathbb{R}^{3}}\left(|\nabla \bar{u}|^{2}+\bar{u}^{2}\right)+\frac{1}{4} \int_{\mathbb{R}^{3}} \rho \phi_{\bar{u}} \bar{u}^{2}-\frac{\mu}{p+1} \int_{\mathbb{R}^{3}} \bar{u}^{p+1}=\bar{c}
$$

and

$$
\int_{\mathbb{R}^{3}}\left(|\nabla \bar{u}|^{2}+\bar{u}^{2}\right)+\int_{\mathbb{R}^{3}} \rho \phi_{\bar{u}} \bar{u}^{2}-\mu \int_{\mathbb{R}^{3}} \bar{u}^{p+1}=0 .
$$

Moreover, since $\bar{u}$ solves (3.1) then, by Lemma 2.2, $\bar{u}$ must also satisfy the Pohozaev equality:

$$
\frac{1}{2} \int_{\mathbb{R}^{3}}|\nabla \bar{u}|^{2}+\frac{3}{2} \int_{\mathbb{R}^{3}} \bar{u}^{2}+\frac{5}{4} \int_{\mathbb{R}^{3}} \rho \phi_{\bar{u}} \bar{u}^{2}+\frac{1}{2} \int_{\mathbb{R}^{3}} \phi_{\bar{u}} \bar{u}^{2}(x, \nabla \rho)-\frac{3 \mu}{p+1} \int_{\mathbb{R}^{3}} \bar{u}^{p+1}=0 .
$$

We now recall that, by assumption, $k \rho(x) \leq(x, \nabla \rho)$ for some $k>\frac{-2(p-2)}{(p-1)}$. Using this in the above equality, we see that

$$
\frac{1}{2} \int_{\mathbb{R}^{3}}\left(|\nabla \bar{u}|^{2}+\bar{u}^{2}\right)+\left(\frac{5+2 k}{4}\right) \int_{\mathbb{R}^{3}} \rho \phi_{\bar{u}} \bar{u}^{2}-\frac{3 \mu}{p+1} \int_{\mathbb{R}^{3}} \bar{u}^{p+1} \leq 0 .
$$

For ease, we now set $\alpha=\|\bar{u}\|_{H^{1}}^{2}, \gamma=\int_{\mathbb{R}^{3}} \rho \phi_{\bar{u}} \bar{u}^{2}$, and $\delta=\mu \int_{\mathbb{R}^{3}} \bar{u}^{p+1}$. From (3.13), (3.14), and (3.15), we can see that $\alpha, \gamma$, and $\delta$ satisfy

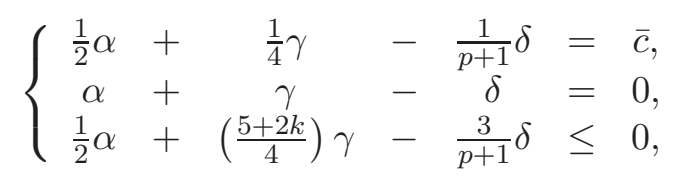

and so, it holds that

$$
\delta \leq \frac{\bar{c}(3+2 k)(p+1)}{2(p-2)+k(p-1)}
$$

and

$$
\alpha=\delta-\gamma
$$

Since $\gamma$ is nonnegative, we find

$$
\alpha \leq \alpha+\gamma=\delta \leq \frac{\bar{c}(3+2 k)(p+1)}{2(p-2)+k(p-1)}
$$

This and (3.12) implies the statement, since $k>\frac{-2(p-2)}{(p-1)}>\frac{-3}{2}$ for $p \in(2,3)$. This concludes the proof. 
Lemma 3.4. If $p \in(2,5), \mu \in\left[\frac{1}{2}, 1\right]$ and $u \in H^{1}\left(\mathbb{R}^{3}\right) \backslash\{0\}$ is a nonnegative solution of (3.8), then, it holds that

$$
I_{\mu}^{\infty}(u) \geq c_{\mu}^{\infty}>0
$$

Moreover, if $p \in(2,5)$ and $u \in H^{1}\left(\mathbb{R}^{3}\right) \backslash\{0\}$ is a nonnegative solution of $(1.13)$, then

$$
I^{\infty}(u) \geq c^{\infty}>0
$$

In both cases, $u>0$.

Proof. The lower bounds follow easily by similar arguments to those used in the proof of Proposition 3.4 in [28]. Since $u$ is nonnegative and nontrivial, then it is strictly positive by the strong maximum principle, and this concludes the proof.

\section{Existence: the CASE of Coercive $\rho(x)$}

In this section we will examine the existence of solutions of (1.1) in the case of a coercive potential $\rho$, namely $\rho(x) \rightarrow+\infty$ as $|x| \rightarrow+\infty$. In the following lemma, we establish that this coercivity is indeed a sufficient condition for the compactness of the embedding $E \hookrightarrow L^{p+1}\left(\mathbb{R}^{3}\right)$.

Lemma 4.1. Assume $\rho(x) \rightarrow+\infty$ as $|x| \rightarrow+\infty$. Then, $E$ is compactly embedded in $L^{p+1}\left(\mathbb{R}^{3}\right)$ for all $p \in(1,5)$.

Proof. We first recall that for any $u \in E$, it holds that

$$
-\Delta \phi_{u}=\rho u^{2}
$$

where $\phi_{u}(x):=\int_{\mathbb{R}^{3}} \frac{\rho(y) u^{2}(y)}{\omega|x-y|} d y \in \mathcal{D}^{1,2}\left(\mathbb{R}^{3}\right)$. Testing this equation with $u_{+}$and $u_{-}$and using the Cauchy-Schwarz inequality, it follows that

$$
\begin{aligned}
\int_{\mathbb{R}^{3}} \rho|u|^{3} & =\int_{\mathbb{R}^{3}} \nabla|u| \nabla \phi_{u} \\
& \leq\left(\left.\int_{\mathbb{R}^{3}}|\nabla| u\right|^{2}\right)^{\frac{1}{2}}\left(\int_{\mathbb{R}^{3}}\left|\nabla \phi_{u}\right|^{2}\right)^{\frac{1}{2}} \\
& =\left(\int_{\mathbb{R}^{3}}|\nabla u|^{2}\right)^{\frac{1}{2}}\left(\int_{\mathbb{R}^{3}} \int_{\mathbb{R}^{3}} \frac{u^{2}(x) \rho(x) u^{2}(y) \rho(y)}{4 \pi|x-y|}\right)^{\frac{1}{2}} \\
& \leq\left(\frac{1}{4 \pi}\right)^{\frac{1}{2}}\|u\|_{E}^{3} .
\end{aligned}
$$

Thus, if $\rho>0$, this implies the continuous embedding $E \hookrightarrow L_{\rho}^{3}\left(\mathbb{R}^{3}\right)$, where $L_{\rho}^{3}\left(\mathbb{R}^{3}\right):=\left\{u: \rho^{\frac{1}{3}} u \in\right.$ $\left.L^{3}\left(\mathbb{R}^{3}\right)\right\}$, equipped with norm $\|u\|_{L_{\rho}^{3}}:=\left\|\rho^{\frac{1}{3}} u\right\|_{L^{3}}$.

Without loss of generality, assume $u_{n} \rightarrow 0$ in E. Since $\rho(x) \rightarrow+\infty$ as $|x| \rightarrow+\infty$, then for any $\epsilon>0$, there exists an $R>0$ such that 


$$
\int_{\mathbb{R}^{3} \backslash B_{R}}\left|u_{n}\right|^{3}=\int_{\mathbb{R}^{3} \backslash B_{R}} \frac{\rho}{\rho}\left|u_{n}\right|^{3}<\epsilon \int_{\mathbb{R}^{3} \backslash B_{R}} \rho\left|u_{n}\right|^{3}<\epsilon C,
$$

for some $C>0$. This and the classical Rellich theorem implies that, passing if necessary to a subsequence,

$$
\int_{\mathbb{R}^{3}}\left|u_{n}\right|^{3} \rightarrow 0
$$

Therefore, we have proved the lemma for $p=2$. Now, if $p \in(1,2)$, then, by interpolation, for some $\alpha \in(0,1)$, it holds that

$$
\left\|u_{n}\right\|_{L^{p+1}\left(\mathbb{R}^{3}\right)} \leq\left\|u_{n}\right\|_{L^{2}\left(\mathbb{R}^{3}\right)}^{\alpha}\left\|u_{n}\right\|_{L^{3}\left(\mathbb{R}^{3}\right)}^{1-\alpha} \rightarrow 0
$$

as the $L^{2}\left(\mathbb{R}^{3}\right)$ norm is bounded. The case $p \in(2,5)$ is similar using Sobolev's inequality, and this concludes the proof.

4.1. Proof of Theorem 2. We are now in position to prove the existence of mountain pass solutions.

Proof of Theorem 2. We first note that by Lemma 3.2, the set $\mathcal{M}$, defined in (3.6), is nonempty.

Claim 1. The values $c_{\mu}$ are critical levels of $I_{\mu}$ for all $\mu \in \mathcal{M}$. Namely, there exists $u \in E$ such that $I_{\mu}(u)=c_{\mu}$ and $I_{\mu}^{\prime}(u)=0$.

By definition, for each $\mu \in \mathcal{M}$, there exists a bounded sequence $\left(u_{n}\right)_{n \in \mathbb{N}} \subset E$ such that $I_{\mu}\left(u_{n}\right) \rightarrow c_{\mu}$ and $I_{\mu}^{\prime}\left(u_{n}\right) \rightarrow 0$. Since $\left(u_{n}\right)_{n \in \mathbb{N}}$ is bounded, there exists $u \in E$ such that, up to a subsequence, $u_{n} \rightarrow u$ in $E$. Using this and the fact that $E$ is compactly embedded in $L^{p+1}\left(\mathbb{R}^{3}\right)$ by Lemma 4.1, arguing as in Lemma 16 in [15], with $V(x)=1$ and $K(x)=\mu$, we see that for all $\delta>0$, there exists a ball $B \subset \mathbb{R}^{3}$ such that

$$
\limsup _{n \rightarrow+\infty} \int_{\mathbb{R}^{3} \backslash B} \rho \phi_{u_{n}} u_{n}^{2}<\delta
$$

and

$$
\limsup _{n \rightarrow+\infty}\left|\int_{\mathbb{R}^{3} \backslash B} \rho \phi_{u_{n}} u_{n} u\right|<\delta .
$$

We then note that since $\left(u_{n}\right)_{n \in \mathbb{N}}$ is bounded in $E$, we also have that, up to a subsequence, $u_{n} \rightarrow u$ in $H^{1}$. Now, using this and the fact that $\left(u_{n}\right)_{n \in \mathbb{N}}$ is a bounded Palais Smale sequence for $I_{\mu}$, as well as (4.3), (4.4), and Lemma 4.1, we can reason as in Lemma 18 in [15], with $V(x)=1$ and $K(x)=\mu$, to see that

$$
\int_{\mathbb{R}^{3}}\left(\left|\nabla u_{n}\right|^{2}+u_{n}^{2}\right) \rightarrow \int_{\mathbb{R}^{3}}\left(|\nabla u|^{2}+u^{2}\right)
$$

Thus, using (4.3) and the boundedness of $\left(u_{n}\right)_{n \in \mathbb{N}}$, we can argue as in the proof of Theorem 1 in [15], to see that 


$$
\int_{\mathbb{R}^{3}} \rho \phi_{u_{n}} u_{n}^{2} \rightarrow \int_{\mathbb{R}^{3}} \rho \phi_{u} u^{2}
$$

which, when combined with (4.5) and Lemma 4.1, implies that

$$
I_{\mu}\left(u_{n}\right) \rightarrow I_{\mu}(u)
$$

Therefore, we have shown

$$
I_{\mu}(u)=c_{\mu}
$$

Moreover, by standard arguments, using the weak convergence $u_{n} \rightarrow u$ in $E$, we can show

$$
I_{\mu}^{\prime}(u)=0
$$

We finally note that, by putting (4.5) and (4.6) together, we have that $\left\|u_{n}\right\|_{E}^{2} \rightarrow\|u\|_{E}^{2}$, and so by Lemma 2.1, it follows that $u_{n} \rightarrow u$ in $E$. This concludes the proof of Claim 1 .

Claim 2. Let $\left(\mu_{n}\right)_{n \in \mathbb{N}}$ be an increasing sequence in $\mathcal{M}$ such that $\mu_{n} \rightarrow 1$ and assume $\left(u_{n}\right)_{n \in \mathbb{N}} \subset E$ is such that $I_{\mu_{n}}\left(u_{n}\right)=c_{\mu_{n}}$ and $I_{\mu_{n}}^{\prime}\left(u_{n}\right)=0$ for each $n$. Then, there exists $u \in E$ such that, up to a subsequence, $u_{n} \rightarrow u$ in $E, I(u)=c$, and $I^{\prime}(u)=0$.

We first note that testing the equation $I_{\mu_{n}}^{\prime}\left(u_{n}\right)=0$ with $\left(u_{n}\right)_{-}$, one sees that $u_{n} \geq 0$ for each $n$. Therefore, it holds that $u_{n}$ satisfies

$$
\begin{gathered}
-\Delta u_{n}+u_{n}+\rho(x) \phi_{u_{n}} u_{n}=\mu_{n} u_{n}^{p}, \\
\frac{1}{2} \int_{\mathbb{R}^{3}}\left(\left|\nabla u_{n}\right|^{2}+u_{n}^{2}\right)+\frac{1}{4} \int_{\mathbb{R}^{3}} \rho \phi_{u_{n}} u_{n}^{2}-\frac{\mu_{n}}{p+1} \int_{\mathbb{R}^{3}} u_{n}^{p+1}=c_{\mu_{n}},
\end{gathered}
$$

and

$$
\int_{\mathbb{R}^{3}}\left(\left|\nabla u_{n}\right|^{2}+u_{n}^{2}\right)+\int_{\mathbb{R}^{3}} \rho \phi_{u_{n}} u_{n}^{2}-\mu_{n} \int_{\mathbb{R}^{3}} u_{n}^{p+1}=0 .
$$

Moreover, since $u_{n}$ solves (4.7) then, using Lemma 2.2 and the assumption $k \rho(x) \leq(x, \nabla \rho)$ for some $k>\frac{-2(p-2)}{(p-1)}$, and arguing as in Lemma 3.3, we see that

$$
\frac{1}{2} \int_{\mathbb{R}^{3}}\left(\left|\nabla u_{n}\right|^{2}+u_{n}^{2}\right)+\left(\frac{5+2 k}{4}\right) \int_{\mathbb{R}^{3}} \rho \phi_{u_{n}} u_{n}^{2}-\frac{3 \mu_{n}}{p+1} \int_{\mathbb{R}^{3}} u_{n}^{p+1} \leq 0 .
$$

Setting $\alpha_{n}=\int_{\mathbb{R}^{3}}\left(\left|\nabla u_{n}\right|^{2}+u_{n}^{2}\right), \gamma_{n}=\int_{\mathbb{R}^{3}} \rho \phi_{u_{n}} u_{n}^{2}$, and $\delta_{n}=\mu_{n} \int_{\mathbb{R}^{3}} u_{n}^{p+1}$, we can see, from (4.8), (4.9), and (4.10), that $\alpha_{n}, \gamma_{n}$, and $\delta_{n}$ satisfy

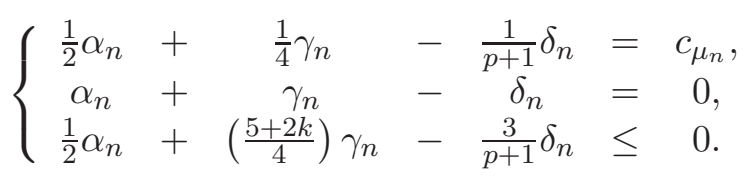


Solving the system, we find that

$$
\begin{gathered}
\delta_{n} \leq \frac{c_{\mu_{n}}(3+2 k)(p+1)}{2(p-2)+k(p-1)}, \\
\gamma_{n} \leq \frac{-2 c_{\mu_{n}}(p-5)}{2(p-2)+k(p-1)},
\end{gathered}
$$

and

$$
\alpha_{n}=\delta_{n}-\gamma_{n}
$$

Since $c_{\mu_{n}}$ is bounded, $k>\frac{-2(p-2)}{(p-1)}>\frac{-3}{2}$, and $\delta_{n}, \gamma_{n}$, and $\alpha_{n}$ are all nonnegative, we can deduce that $\delta_{n}, \gamma_{n}$, and $\alpha_{n}$ are all bounded. Hence, the sequence $\left(u_{n}\right)_{n \in \mathbb{N}}$ is bounded in $E$ and so there exists $u \in E$ such that, up to a subsequence, $u_{n} \rightarrow u$ in $E$.

We now follow a similar procedure to that of Claim 1. Using the facts that $I_{\mu_{n}}^{\prime}\left(u_{n}\right)=0, u_{n}$ is bounded in $E, E$ is compactly embedded in $L^{p+1}\left(\mathbb{R}^{3}\right)$ by Lemma 4.1 , and $\mu_{n} \rightarrow 1$, by an easy argument similar to the proof of Lemma 16 in [15], with $V(x)=1$ and $K(x)=\mu_{n}$, we have that for all $\delta>0$, there exists a ball $B \subset \mathbb{R}^{3}$ such that

$$
\limsup _{n \rightarrow+\infty} \int_{\mathbb{R}^{3} \backslash B} \rho \phi_{u_{n}} u_{n}^{2}<\delta
$$

and

$$
\limsup _{n \rightarrow+\infty}\left|\int_{\mathbb{R}^{3} \backslash B} \rho \phi_{u_{n}} u_{n} u\right|<\delta .
$$

Now, using the facts that $I_{\mu_{n}}^{\prime}\left(u_{n}\right)=0$ and $\mu_{n} \rightarrow 1$, as well as (4.12), (4.13), and Lemma 4.1, we can adapt the proof of Lemma 18 in [15], with $V(x)=1$ and $K(x)=\mu_{n}$, to see that

$$
\int_{\mathbb{R}^{3}}\left(\left|\nabla u_{n}\right|^{2}+u_{n}^{2}\right) \rightarrow \int_{\mathbb{R}^{3}}\left(|\nabla u|^{2}+u^{2}\right) .
$$

Finally, using (4.12), (4.14), the boundedness of $u_{n}$, Lemma 4.1, and the fact that $\mu_{n} \rightarrow 1$, we can easily adapt the proof of Theorem 1 in [15], to see that

$$
\begin{gathered}
\int_{\mathbb{R}^{3}} \rho \phi_{u_{n}} u_{n}^{2} \rightarrow \int_{\mathbb{R}^{3}} \rho \phi_{u} u^{2}, \\
c_{\mu_{n}}=I_{\mu_{n}}\left(u_{n}\right) \rightarrow I(u),
\end{gathered}
$$

and

$$
0=I_{\mu_{n}}^{\prime}\left(u_{n}\right) \rightarrow I^{\prime}(u) .
$$


As in Claim 1, we see that (4.14) and (4.15) imply that $\left\|u_{n}\right\|_{E}^{2} \rightarrow\|u\|_{E}^{2}$, and so by Lemma 2.1, it follows that $u_{n} \rightarrow u$ in $E$. We now recall that, for $p \in(2,3)$, it holds that $c_{\mu_{n}} \rightarrow c$ as $\mu_{n} \nearrow 1$ by definition (3.7). Thus, from (4.16) it follows that $I(u)=c$.

Conclusion. Let $\left(\mu_{n}\right)_{n \in \mathbb{N}}$ be an increasing sequence in $\mathcal{M}$ such that $\mu_{n} \rightarrow 1$. By Claim 1, we can choose $\left(u_{n}\right)_{n \in \mathbb{N}} \subset E$ such that $I_{\mu_{n}}\left(u_{n}\right)=c_{\mu_{n}}$ and $I_{\mu_{n}}^{\prime}\left(u_{n}\right)=0$ for each $n$. By Claim 2, it follows that that there exists $u \in E$ such that, up to a subsequence, $u_{n} \rightarrow u$ in $E, I(u)=c$, and $I^{\prime}(u)=0$. Namely, we have shown $\left(u, \phi_{u}\right) \in E\left(\mathbb{R}^{3}\right) \times D^{1,2}\left(\mathbb{R}^{3}\right)$ is a solution of (1.1). By the strong maximum principle $\phi_{u}$ is strictly positive. Testing the equation $I^{\prime}(u)=0$ with $u_{-}$one sees that $u \geq 0$ and, in fact, strictly positive as a consequence of the strong maximum principle. This concludes the proof.

4.2. Proof of Corollary 1. In the next proof, we show the existence of least energy solutions.

Proof of Corollary 1. When $p>3$ it is standard to see that the Mountain Pass level $c$ has the following characterisation

$$
c=\inf _{u \in \mathcal{N}} I(u), \quad \mathcal{N}=\left\{u \in E \backslash\{0\} \mid I^{\prime}(u) u=0\right\}
$$

see e.g. Theorem 5 in [15]. It follows that the mountain pass solution $u$ found in Theorem 1 is a least energy solutions of $I$ in this case. If $p \in(2,3]$, define

$$
c^{*}:=\inf _{u \in \mathcal{A}} I(u), \text { where } \mathcal{A}:=\left\{u \in E\left(\mathbb{R}^{3}\right) \backslash\{0\}: u \text { is a nonnegative solution to }(1.9)\right\} \text {. }
$$

When $p=3$, we notice that the mountain pass critical point, $u$, that we found in Theorem 1 is such that $u \in \mathcal{A}$. Similarly, when $p \in(2,3)$, the mountain pass critical point that we found in Theorem 2 is in $\mathcal{A}$. Therefore, in both cases, $\mathcal{A}$ is nonempty and $c^{*}$ is well-defined. Now, let $\left(w_{n}\right)_{n \in \mathbb{N}} \subset \mathcal{A}$ be a minimising sequence for $I$ on $\mathcal{A}$, namely $I\left(w_{n}\right) \rightarrow c^{*}$ as $n \rightarrow+\infty$ and $I^{\prime}\left(w_{n}\right)=0$. If $p=3$, it holds that

$$
c+1 \geq(p+1) I\left(w_{n}\right)-I^{\prime}\left(w_{n}\right) w_{n} \geq\left\|w_{n}\right\|_{H^{1}\left(\mathbb{R}^{3}\right)}^{2},
$$

and so it follows that $\left(w_{n}\right)_{n \in \mathbb{N}}$ is bounded. If $p \in(2,3)$, setting $\alpha_{n}=\int_{\mathbb{R}^{3}}\left(\left|\nabla w_{n}\right|^{2}+w_{n}^{2}\right), \gamma_{n}=$ $\int_{\mathbb{R}^{3}} \rho \phi_{w_{n}} w_{n}^{2}$, and $\delta_{n}=\int_{\mathbb{R}^{3}} w_{n}^{p+1}$, and arguing as in Theorem 2 Claim 2, we see that $\alpha_{n}, \gamma_{n}$, and $\delta_{n}$ satisfy the system (4.11) with $d_{n}:=I\left(w_{n}\right)$ in the place of $c_{\mu_{n}}$. Thus, solving this system and arguing as in Theorem 2 Claim 2, we can obtain that $\alpha_{n}, \gamma_{n}$, and $\delta_{n}$ are all bounded since $\left(d_{n}\right)_{n \in \mathbb{N}}$ is uniformly bounded. It follows that $\left(w_{n}\right)_{n \in \mathbb{N}}$ is also bounded in this case. Therefore, for all $p \in(2,3]$, there exists $w_{0} \in E$ such that, up to a subsequence, $w_{n} \rightarrow w_{0}$ in $E$. Arguing as in the proof of Theorem 2 Claim 1, we can show $w_{n} \rightarrow w_{0}$ in $E, I\left(w_{0}\right)=c^{*}$, and $I^{\prime}\left(w_{0}\right)=0$. We note that by Lemma 3.3, it holds that $c^{*} \geq C>0$ for some uniform constant $C>0$, and so $w_{0}$ is nontrivial. Finally, reasoning as in the conclusion of Theorem 2, we see that both $w_{0}, \phi_{w_{0}}$ are positive, and this concludes the proof. 


\section{Existence: The CASE OF NON-COERCIVE $\rho(x)$}

We now turn our attention to the problem of finding solutions when $\rho$ is non-coercive, namely when $\rho(x) \rightarrow \rho_{\infty}>0$ as $|x| \rightarrow+\infty$. In this setting, $E\left(\mathbb{R}^{3}\right)$ coincides with the larger space $H^{1}\left(\mathbb{R}^{3}\right)$, and so we look for solutions $\left(u, \phi_{u}\right) \in H^{1}\left(\mathbb{R}^{3}\right) \times D^{1,2}\left(\mathbb{R}^{3}\right)$ of $(1.1)$.

5.1. Bounded PS sequences: proof of Proposition 2. Before moving forward, we will need some useful preliminary lemmas.

Lemma 5.1 ([42]). Let $p \geq 0$ and $\left(u_{n}\right)_{n \in \mathbb{N}} \subset L^{p+1}\left(\mathbb{R}^{3}\right)$ be such that $u_{n} \rightarrow u$ almost everywhere on $\mathbb{R}^{3}, \sup _{n}\left\|u_{n}\right\|_{L^{p+1}}<+\infty$, and $\left(u_{n}\right)_{-} \rightarrow 0$ in $L^{p+1}\left(\mathbb{R}^{3}\right)$. Then, $u \in L^{p+1}\left(\mathbb{R}^{3}\right), u \geq 0$,

$$
\left(u_{n}-u\right)_{-} \rightarrow 0 \quad \text { in } L^{p+1}\left(\mathbb{R}^{3}\right),
$$

and

$$
\left\|\left(u_{n}-u\right)_{+}\right\|_{L^{p+1}}^{p+1}=\left\|\left(u_{n}\right)_{+}\right\|_{L^{p+1}}^{p+1}-\left\|u_{+}\right\|_{L^{p+1}}^{p+1}+o(1) .
$$

Lemma 5.2. Let $p>0$ and set

$$
F(u)=\frac{1}{p+1} \int_{\mathbb{R}^{3}}|u|^{p+1}, \quad F_{+}(u)=\frac{1}{p+1} \int_{\mathbb{R}^{3}} u_{+}^{p+1} .
$$

Assume $\left(u_{n}\right)_{n \in \mathbb{N}} \subset H^{1}\left(\mathbb{R}^{3}\right)$ is such that $u_{n} \rightarrow u$ almost everywhere on $\mathbb{R}^{3}$ and $\sup _{n}\left\|u_{n}\right\|_{H^{1}}<+\infty$. Then, it holds that

$$
F^{\prime}\left(u_{n}\right)-F^{\prime}\left(u_{n}-u\right)-F^{\prime}(u)=o(1), \quad \text { in } H^{-1}\left(\mathbb{R}^{3}\right)
$$

If, in addition, $\left(u_{n}\right)_{-} \rightarrow 0$ in $L^{p+1}\left(\mathbb{R}^{3}\right)$, then

$$
F_{+}^{\prime}\left(u_{n}\right)-F_{+}^{\prime}\left(u_{n}-u\right)-F_{+}^{\prime}(u)=o(1), \quad \text { in } H^{-1}\left(\mathbb{R}^{3}\right) .
$$

Proof. The result follows as a consequence of Lemma 3.2 in [42], Lemma 5.1, and Hölder's inequality.

The final preliminary result that we need is a splitting lemma for the nonlocal part of the derivative of the energy functional along bounded sequences. The proof follows by convexity estimates and Fatou's lemma, adapting similar arguments of Section 3 in [41] and Lemma 4.2 in [23] to a nonlocal context.

Lemma 5.3. [Nonlocal splitting lemma] Assume $\left(u_{n}\right)_{n \in \mathbb{N}} \subset H^{1}\left(\mathbb{R}^{3}\right)$ is bounded and $u_{n} \rightarrow v_{0}$ almost everywhere. Suppose further $\rho \in C\left(\mathbb{R}^{3}\right)$ is nonnegative and $\rho(x) \rightarrow \rho_{\infty} \geq 0$ as $|x| \rightarrow+\infty$. Then, the following hold:

(i) $\rho \phi_{\left(u_{n}-v_{0}\right)}\left(u_{n}-v_{0}\right)-\rho_{\infty} \bar{\phi}_{\left(u_{n}-v_{0}\right)}\left(u_{n}-v_{0}\right)=o(1)$ in $H^{-1}\left(\mathbb{R}^{3}\right)$

(ii) $\rho \phi_{u_{n}} u_{n}-\rho \phi_{\left(u_{n}-v_{0}\right)}\left(u_{n}-v_{0}\right)-\rho \phi_{v_{0}} v_{0}=o(1)$ in $H^{-1}\left(\mathbb{R}^{3}\right)$. 
Proof. For the proof of $(i)$, we set

$$
\phi_{u}^{*}(x):=\int_{\mathbb{R}^{3}} \frac{u^{2}(u)}{4 \pi|x-y|} \mathrm{d} y .
$$

Take any $h \in H^{1}$, and note that

$$
\begin{aligned}
\left|\int_{\mathbb{R}^{3}}\left(\rho \phi_{\left(u_{n}-v_{0}\right)}\left(u_{n}-v_{0}\right)-\rho_{\infty} \bar{\phi}_{\left(u_{n}-v_{0}\right)}\left(u_{n}-v_{0}\right)\right) h\right| \leq & \left|\int_{\mathbb{R}^{3}}\left(\rho-\rho_{\infty}\right) \phi_{\left(u_{n}-v_{0}\right)}\left(u_{n}-v_{0}\right) h\right| \\
& +\left|\int_{\mathbb{R}^{3}} \rho_{\infty}\left(\phi_{\left(u_{n}-v_{0}\right)}-\bar{\phi}_{\left(u_{n}-v_{0}\right)}\right)\left(u_{n}-v_{0}\right) h\right| \\
& =: I_{1}+I_{2} .
\end{aligned}
$$

Now, by assumption, for every $\epsilon>0$, there exists $R_{\epsilon}>0$ such that $\left|\rho-\rho_{\infty}\right|<\epsilon$ for all $|x|>R_{\epsilon}$. So, using Hölder's and Sobolev's inequalities, we can see that

$$
\begin{aligned}
I_{1} & \leq\left|\int_{B_{R_{\epsilon}}}\left(\rho-\rho_{\infty}\right) \phi_{\left(u_{n}-v_{0}\right)}\left(u_{n}-v_{0}\right) h\right|+\left|\int_{|x|>R_{\epsilon}}\left(\rho-\rho_{\infty}\right) \phi_{\left(u_{n}-v_{0}\right)}\left(u_{n}-v_{0}\right) h\right| \\
& \leq\|\rho\|_{L^{\infty}}\left\|\phi_{\left(u_{n}-v_{0}\right)}\right\|_{L^{6}}\left\|u_{n}-v_{0}\right\|_{L^{2}\left(B_{R_{\epsilon}}\right)}\|h\|_{L^{3}}+\left.\epsilon\left\|\phi_{\left(u_{n}-v_{0}\right)}\right\|\right|_{L^{6}}\left\|u_{n}-v_{0}\right\|_{L^{2}}\|h\|_{L^{3}} \\
& \lesssim\left(\|\rho\|_{L^{\infty}}\left\|\nabla \phi_{\left(u_{n}-v_{0}\right)}\right\|_{L^{2}}\left\|u_{n}-v_{0}\right\|_{L^{2}\left(B_{R_{\epsilon}}\right)}+\left.\epsilon\left\|\nabla \phi_{\left(u_{n}-v_{0}\right)}\right\|\right|_{L^{2}}\left\|u_{n}-v_{0}\right\|_{L^{2}}\right)\|h\|_{H^{1}} .
\end{aligned}
$$

Moreover, by using Hölder's and Sobolev's inequalities once again, we have

$$
\begin{aligned}
I_{2} & \leq \rho_{\infty}\left\|\phi_{\left(u_{n}-v_{0}\right)}-\bar{\phi}_{\left(u_{n}-v_{0}\right)} \mid\right\|_{L^{6}}\left\|u_{n}-v_{0}\right\|_{L^{2}}\|h\|_{L^{3}} \\
& \lesssim \rho_{\infty}\left\|\phi_{\left(u_{n}-v_{0}\right)}-\bar{\phi}_{\left(u_{n}-v_{0}\right)}\right\|_{L^{6}}\left\|u_{n}-v_{0}\right\|_{L^{2}}\|h\|_{H^{1}}
\end{aligned}
$$

and, by Minkowski's, Sobolev's, and Hardy-Littlewood-Sobolev inequalities, for every $\epsilon>0$, it holds

$$
\begin{aligned}
\left\|\phi_{\left(u_{n}-v_{0}\right)}-\bar{\phi}_{\left(u_{n}-v_{0}\right)}\right\|_{L^{6}} & =\left(\int_{\mathbb{R}^{3}}\left|\int_{\mathbb{R}^{3}} \frac{\left(\rho(y)-\rho_{\infty}\right)\left(u_{n}-v_{0}\right)^{2}(y)}{4 \pi|x-y|} \mathrm{d} y\right|^{6} \mathrm{~d} x\right)^{\frac{1}{6}} \\
& \leq\left(\int_{\mathbb{R}^{3}}\left(\int_{B_{R_{\epsilon}}} \frac{\left|\rho(y)-\rho_{\infty}\right|\left(u_{n}-v_{0}\right)^{2}(y)}{4 \pi|x-y|} \mathrm{d} y\right)^{6} \mathrm{~d} x\right)^{\frac{1}{6}} \\
& +\left(\int_{\mathbb{R}^{3}}\left(\int_{|x|>R_{\epsilon}} \frac{\left|\rho(y)-\rho_{\infty}\right|\left(u_{n}-v_{0}\right)^{2}(y)}{4 \pi|x-y|} \mathrm{d} y\right)^{6} \mathrm{~d} x\right)^{\frac{1}{6}} \\
& \leq\|\rho\|_{L^{\infty}}\left(\int_{\mathbb{R}^{3}}\left(\int_{\mathbb{R}^{3}} \frac{\left(u_{n}-v_{0}\right)^{2}(y) \chi_{B_{R_{\epsilon}}}(y)}{4 \pi|x-y|} \mathrm{d} y\right)^{6} \mathrm{~d} x\right)^{\frac{1}{6}}
\end{aligned}
$$




$$
\begin{aligned}
&+\epsilon\left(\int_{\mathbb{R}^{3}}\left(\int_{\mathbb{R}^{3}} \frac{\left(u_{n}-v_{0}\right)^{2}(y)}{4 \pi|x-y|} \mathrm{d} y\right)^{6} \mathrm{~d} x\right)^{\frac{1}{6}} \\
&=\|\rho\|_{L^{\infty}}\left\|\phi_{\left(u_{n}-v_{0}\right) \chi_{B_{R_{\epsilon}}}}\right\|\left\|_{L^{6}}+\epsilon\right\| \phi_{\left(u_{n}-v_{0}\right)}^{*} \|_{L^{6}} \\
& \lesssim\|\rho\|_{L^{\infty}}\left\|\nabla \phi_{\left(u_{n}-v_{0}\right) \chi_{B_{R_{\epsilon}}}^{*}}\right\|_{L^{2}}+\epsilon\left\|\nabla \phi_{\left(u_{n}-v_{0}\right)}^{*}\right\|_{L^{2}} \\
& \lesssim\|\rho\|_{L^{\infty}}\left\|\left(u_{n}-v_{0}\right) \chi_{B_{R_{\epsilon}}}\right\|_{L^{\frac{12}{5}}}^{2}+\epsilon\left\|\nabla \phi_{\left(u_{n}-v_{0}\right)}^{*}\right\|_{L^{2}} .
\end{aligned}
$$

So, putting together (5.1), (5.2), (5.3), and (5.4), we obtain, for every $\epsilon>0$,

$$
\begin{aligned}
\mid \int_{\mathbb{R}^{3}} & \left(\rho \phi_{\left(u_{n}-v_{0}\right)}\left(u_{n}-v_{0}\right)-\rho_{\infty} \bar{\phi}_{\left(u_{n}-v_{0}\right)}\left(u_{n}-v_{0}\right)\right) h \mid \\
\leq & C\left(\left.\|\rho\|\right|_{L^{\infty}}\left\|\nabla \phi_{\left(u_{n}-v_{0}\right)}||_{L^{2}}\right\| u_{n}-v_{0}\left\|\left.\right|_{L^{2}\left(B_{R_{\epsilon}}\right)}+\epsilon\right\| \nabla \phi_{\left(u_{n}-v_{0}\right)}\left\|\left.\right|_{L^{2}}\right\| u_{n}-v_{0} \|_{L^{2}}\right. \\
\quad & \quad \rho_{\infty}\|\rho\|_{L^{\infty}}\left\|\left(u_{n}-v_{0}\right) \chi_{B_{R_{\epsilon}}}\right\|_{L^{\frac{12}{5}}}^{2}\left\|u_{n}-v_{0}\right\|_{L^{2}}+\rho_{\infty} \epsilon\left\|\nabla \phi_{\left(u_{n}-v_{0}\right)}^{*}\left|\left\|_{L^{2}}\right\| u_{n}-v_{0} \|\right|_{L^{2}}\right)\|h\|_{H^{1}}
\end{aligned}
$$

for some $C>0$. Since $\rho \in L^{\infty}, \phi_{\left(u_{n}-v_{0}\right)}, \phi_{\left(u_{n}-v_{0}\right)}^{*}$ are uniformly bounded in $D^{1,2}, u_{n}-v_{0}$ is uniformly bounded in $L^{2}$, and $u_{n}-v_{0} \rightarrow 0$ in $L_{\text {loc }}^{2}$ and $L_{\text {loc }}^{\frac{12}{5}}$, then we have proven $(i)$.

To prove (ii), we first take any $h \in H^{1}$, and note that by Hölder's and Sobolev's inequalities, it holds that

$$
\begin{aligned}
\mid \int_{\mathbb{R}^{3}}\left(\rho \phi_{u_{n}} u_{n}\right. & \left.-\rho \phi_{\left(u_{n}-v_{0}\right)}\left(u_{n}-v_{0}\right)-\rho \phi_{v_{0}} v_{0}\right) h \mid \\
& \leq\|\rho\|_{L^{\infty}}\left\|\phi_{u_{n}} u_{n}-\phi_{\left(u_{n}-v_{0}\right)}\left(u_{n}-v_{0}\right)-\phi_{v_{0}} v_{0}\right\|_{L^{\frac{3}{2}}}\|h\|_{L^{3}} \\
& \leq C\|\rho\|_{L^{\infty}}\left\|\phi_{u_{n}} u_{n}-\phi_{\left(u_{n}-v_{0}\right)}\left(u_{n}-v_{0}\right)-\phi_{v_{0}} v_{0}\right\|_{L^{\frac{3}{2}}}\|h\|_{H^{1}}
\end{aligned}
$$

for some $C>0$. Now, by convexity, iterating the inequality

$$
|a+b|^{\frac{3}{2}} \leq \sqrt{2}\left(|a|^{\frac{3}{2}}+|b|^{\frac{3}{2}}\right),
$$

we can obtain

$$
\begin{aligned}
F_{n} & :=\left|\phi_{u_{n}} u_{n}-\phi_{\left(u_{n}-v_{0}\right)}\left(u_{n}-v_{0}\right)-\phi_{v_{0}} v_{0}\right|^{\frac{3}{2}} \\
& \leq 2\left(\left|\left(\phi_{u_{n}}-\phi_{\left(u_{n}-v_{0}\right)}\right) u_{n}\right|^{\frac{3}{2}}+\left|\phi_{\left(u_{n}-v_{0}\right)} v_{0}\right|^{\frac{3}{2}}+\left|\phi_{v_{0}} v_{0}\right|^{\frac{3}{2}}\right) .
\end{aligned}
$$

Then, using the Cauchy-Schwarz inequality, we notice that

$$
\begin{aligned}
\mid\left(\phi_{u_{n}}-\phi_{\left(u_{n}-v_{0}\right)} \mid\right. & \leq \int_{\mathbb{R}^{3}} \frac{\rho\left|2 u_{n}-v_{0}\right|\left|v_{0}\right|}{4 \pi|x-y|} \mathrm{d} y \\
& \leq\left(\int_{\mathbb{R}^{3}} \frac{\rho\left|2 u_{n}-v_{0}\right|^{2}}{4 \pi|x-y|} \mathrm{d} y\right)^{\frac{1}{2}}\left(\int_{\mathbb{R}^{3}} \frac{\rho\left|v_{0}\right|^{2}}{4 \pi|x-y|} \mathrm{d} y\right)^{\frac{1}{2}}
\end{aligned}
$$




$$
=\phi_{\left(2 u_{n}-v_{0}\right)}^{\frac{1}{2}} \phi_{v_{0}}^{\frac{1}{2}}
$$

and so, using this and applying Young's inequality twice, we see that, for every $\epsilon>0$,

$$
\begin{aligned}
\left|\left(\phi_{u_{n}}-\phi_{\left(u_{n}-v_{0}\right)}\right) u_{n}\right|^{\frac{3}{2}} & \leq \phi_{\left(2 u_{n}-v_{0}\right)}^{\frac{3}{4}} \phi_{v_{0}}^{\frac{3}{4}}\left|u_{n}\right|^{\frac{3}{2}} \\
& \leq \epsilon^{\frac{8}{7}} \phi_{\left(2 u_{n}-v_{0}\right)}^{\frac{6}{7}}\left|u_{n}\right|^{\frac{12}{7}}+\epsilon^{-8} \phi_{v_{0}}^{6} \\
& \leq \epsilon^{\frac{8}{7}}\left(\phi_{\left(2 u_{n}-v_{0}\right)}^{6}+\left|u_{n}\right|^{2}\right)+\epsilon^{-8} \phi_{v_{0}}^{6} .
\end{aligned}
$$

Moreover, again using Young's inequality, it holds, for every $\epsilon>0$,

$$
\left|\phi_{\left(u_{n}-v_{0}\right)} v_{0}\right|^{\frac{3}{2}} \leq \epsilon^{4} \phi_{\left(u_{n}-v_{0}\right)}^{6}+\epsilon^{-\frac{4}{3}} v_{0}^{2}
$$

Combining (5.6), (5.7), and (5.8), we see that, for all $\epsilon>0$,

$$
F_{n} \leq 2\left(\epsilon^{\frac{8}{7}}\left(\phi_{\left(2 u_{n}-v_{0}\right)}^{6}+\left|u_{n}\right|^{2}\right)+\epsilon^{-8} \phi_{v_{0}}^{6}+\epsilon^{4} \phi_{\left(u_{n}-v_{0}\right)}^{6}+\epsilon^{-\frac{4}{3}} v_{0}^{2}+\left|\phi_{v_{0}} v_{0}\right|^{\frac{3}{2}}\right)=: G_{n}
$$

and so $G_{n}-F_{n} \geq 0$. We recall that by assumption $u_{n} \rightarrow v_{0}$ almost everywhere, and so it follows that $\phi_{\left(u_{n}-v_{0}\right)} \rightarrow 0, \phi_{u_{n}} \rightarrow \phi_{v_{0}}$, and $\phi_{\left(2 u_{n}-v_{0}\right)} \rightarrow \phi_{v_{0}}$ almost everywhere. Thus, applying Fatou's Lemma to $G_{n}-F_{n}$, we obtain

$$
\begin{aligned}
& 2 \int_{\mathbb{R}^{3}}\left(\epsilon^{\frac{8}{7}}\left(\phi_{v_{0}}^{6}+\left|v_{0}\right|^{2}\right)+\epsilon^{-8} \phi_{v_{0}}^{6}+\epsilon^{-\frac{4}{3}} v_{0}^{2}+\left|\phi_{v_{0}} v_{0}\right|^{\frac{3}{2}}\right) \\
& \leq 2\left(\epsilon^{\frac{8}{7}} \sup _{n \geq 1} \int_{\mathbb{R}^{3}}\left(\phi_{\left(2 u_{n}-v_{0}\right)}^{6}+\left|u_{n}\right|^{2}\right)+\epsilon^{-8} \int_{\mathbb{R}^{3}} \phi_{v_{0}}^{6}+\epsilon^{4} \sup _{n \geq 1} \int_{\mathbb{R}^{3}} \phi_{\left(u_{n}-v_{0}\right)}^{6}+\epsilon^{-\frac{4}{3}} \int_{\mathbb{R}^{3}} v_{0}^{2}\right. \\
& \left.\quad+\int_{\mathbb{R}^{3}}\left|\phi_{v_{0}} v_{0}\right|^{\frac{3}{2}}\right)-\limsup _{n \rightarrow+\infty} \int_{\mathbb{R}^{3}} F_{n}
\end{aligned}
$$

Therefore, after cancelations and using Sobolev's inequality, we see that

$$
\begin{aligned}
& \limsup _{n \rightarrow+\infty} \int_{\mathbb{R}^{3}} F_{n} \leq 2\left(\epsilon^{\frac{8}{7}} \sup _{n \geq 1} \int_{\mathbb{R}^{3}}\left(\phi_{\left(2 u_{n}-v_{0}\right)}^{6}+\left|u_{n}\right|^{2}\right)+\epsilon^{4} \sup _{n \geq 1} \int_{\mathbb{R}^{3}} \phi_{\left(u_{n}-v_{0}\right)}^{6}-\epsilon^{\frac{8}{7}} \int_{\mathbb{R}^{3}}\left(\phi_{v_{0}}^{6}+\left|v_{0}\right|^{2}\right)\right) \\
&=2\left(\epsilon^{\frac{8}{7}} \sup _{n \geq 1}\left(\left\|\phi_{\left(2 u_{n}-v_{0}\right)}\right\|_{L^{6}}^{6}+\left\|u_{n}\right\|_{L^{2}}^{2}\right)+\epsilon^{4} \sup _{n \geq 1}\left\|\phi_{\left(u_{n}-v_{0}\right)}\right\|_{L^{6}}^{6}\right. \\
&\left.\quad-\epsilon^{\frac{8}{7}}\left(\left\|\phi_{v_{0}}\right\|_{L^{6}}^{6}+\left\|v_{0}\right\|_{L^{2}}^{2}\right)\right) \\
& \leq C\left(\epsilon^{\frac{8}{7}} \sup _{n \geq 1}\left(\left\|\nabla \phi_{\left(2 u_{n}-v_{0}\right)}\right\|_{L^{2}}^{6}+\left\|u_{n}\right\|_{L^{2}}^{2}\right)+\epsilon^{4} \sup _{n \geq 1}\left\|\nabla \phi_{\left(u_{n}-v_{0}\right)}\right\|_{L^{2}}^{6}\right. \\
& \\
&\left.\quad-\epsilon^{\frac{8}{7}}\left(\left\|\phi_{v_{0}}\right\|_{L^{6}}^{6}+\left\|v_{0}\right\|_{L^{2}}^{2}\right)\right),
\end{aligned}
$$


for some $C>0$ and for all $\epsilon>0$. We note that $u_{n}, v_{0}$ are uniformly bounded in $L^{2}$ and $\phi_{\left(u_{n}-v_{0}\right)}, \phi_{\left(2 u_{n}-v_{0}\right)}$ are uniformly bounded in $D^{1,2}$ since $u_{n}-v_{0}, 2 u_{n}-v_{0}$ are uniformly bounded in $H^{1}$. Moreover, since $v_{0} \in H^{1}$, it follows that $\left\|\phi_{v_{0}}\right\|_{L^{6}}^{6}$ is bounded by Sobolev's inequality. Hence, since $\epsilon>0$ is arbitrary, it holds that

$$
\lim _{n \rightarrow+\infty} \int_{\mathbb{R}^{3}} F_{n}=0
$$

which combined with (5.5) yields $(i i)$, and this concludes the proof.

With these preliminaries in place, we now prove a useful 'splitting' proposition for bounded Palais-Smale sequences for $I_{\mu}$, that highlights the connection to the problem at infinity.

Proof of Proposition 2. Since $\left(u_{n}\right)_{n \in \mathbb{N}}$ is bounded in $H^{1}$, we may assume $u_{n} \rightarrow v_{0}$ in $H^{1}$ and $u_{n} \rightarrow v_{0}$ a.e. in $\mathbb{R}^{3}$. We set $u_{n}^{1}:=u_{n}-v_{0}$, and we first note that

$$
\left\|u_{n}^{1}\right\|_{H^{1}}^{2}=\left\|u_{n}-v_{0}\right\|_{H^{1}}^{2}=\left\|u_{n}\right\|_{H^{1}}^{2}-\left\|v_{0}\right\|_{H^{1}}^{2}+o(1)
$$

We now prove three claims involving the sequence $\left(u_{n}^{1}\right)_{n \in \mathbb{N}}$.

Claim 1. $I_{\mu}^{\infty}\left(u_{n}^{1}\right)=I_{\mu}\left(u_{n}\right)-I_{\mu}\left(v_{0}\right)+o(1)$.

Testing $I_{\mu}^{\prime}\left(u_{n}\right)$ with $\left(u_{n}\right)_{-}$we have

$$
\begin{aligned}
I_{\mu}^{\prime}\left(u_{n}\right)\left(u_{n}\right)_{-} & =\int_{\mathbb{R}^{3}}\left(\nabla u_{n} \nabla\left(\left(u_{n}\right)_{-}\right)+u_{n}\left(u_{n}\right)_{-}\right)+\int_{\mathbb{R}^{3}} \rho \phi_{u_{n}} u_{n}\left(u_{n}\right)_{-}-\mu \int_{\mathbb{R}^{3}}\left(u_{n}\right)_{+}^{p}\left(u_{n}\right)_{-} \\
& =\left\|\left(u_{n}\right)_{-}\right\|_{H^{1}}^{2}+\int_{\mathbb{R}^{3}} \rho \phi_{u_{n}}\left(u_{n}\right)_{-}^{2} .
\end{aligned}
$$

Since $\left(u_{n}\right)_{n \in \mathbb{N}}$ is bounded, $I_{\mu}^{\prime}\left(u_{n}\right)\left(u_{n}\right)_{-}=o(1)$, which implies

$$
\left(u_{n}\right)_{-} \rightarrow 0 \text { in } H^{1}
$$

and by Sobolev's embedding

$$
\left(u_{n}\right)_{-} \rightarrow 0 \text { in } L^{p+1} \forall p \in[1,5]
$$

Now, using this and the boundedness of $\left(u_{n}\right)_{n \in \mathbb{N}}$ in $L^{p+1}$, it holds, by Lemma 5.1, that

$$
\left\|\left(u_{n}^{1}\right)_{+}\right\|_{L^{p+1}}^{p+1}=\left\|\left(u_{n}\right)_{+}\right\|_{L^{p+1}}^{p+1}-\left\|\left(v_{0}\right)_{+}\right\|_{L^{p+1}}^{p+1}+o(1) .
$$

Therefore, using this and (5.9), we can see that

$$
I_{\mu}^{\infty}\left(u_{n}^{1}\right)=\frac{1}{2}\left(\left\|u_{n}\right\|_{H^{1}}^{2}-\left\|v_{0}\right\|_{H^{1}}^{2}\right)+\frac{1}{4} \int_{\mathbb{R}^{3}} \rho_{\infty} \bar{\phi}_{\left(u_{n}-v_{0}\right)}\left(u_{n}-v_{0}\right)^{2}
$$




$$
-\frac{\mu}{p+1}\left(\left\|\left(u_{n}\right)_{+}\right\|_{L^{p+1}}^{p+1}-\left\|\left(v_{0}\right)_{+}\right\|_{L^{p+1}}^{p+1}\right)+o(1) .
$$

We now notice that since, by symmetry,

$$
\int_{\mathbb{R}^{3}} \rho_{\infty}\left(u_{n}-v_{0}\right)^{2} \phi_{\left(u_{n}-v_{0}\right)}=\int_{\mathbb{R}^{3}} \rho\left(u_{n}-v_{0}\right)^{2} \bar{\phi}_{\left(u_{n}-v_{0}\right)},
$$

then it holds that

$$
\begin{aligned}
\left|\int_{\mathbb{R}^{3}} \rho \phi_{\left(u_{n}-v_{0}\right)}\left(u_{n}-v_{0}\right)^{2}-\int_{\mathbb{R}^{3}} \rho_{\infty} \bar{\phi}_{\left(u_{n}-v_{0}\right)}\left(u_{n}-v_{0}\right)^{2}\right| \leq & \int_{\mathbb{R}^{3}} \phi_{\left(u_{n}-v_{0}\right)}\left(u_{n}-v_{0}\right)^{2}\left|\rho(x)-\rho_{\infty}\right| \\
& +\int_{\mathbb{R}^{3}} \bar{\phi}_{\left(u_{n}-v_{0}\right)}\left(u_{n}-v_{0}\right)^{2}\left|\rho(x)-\rho_{\infty}\right| \\
& =: I_{1}+I_{2} .
\end{aligned}
$$

We note that for all $\epsilon>0$ there exists $R_{\epsilon}>0$ such that $\left|\rho(x)-\rho_{\infty}\right|<\epsilon$ for all $|x|>R_{\epsilon}$. Thus, we can see,

$$
\begin{aligned}
I_{1} & \leq \int_{B_{R_{\epsilon}}} \phi_{\left(u_{n}-v_{0}\right)}\left(u_{n}-v_{0}\right)^{2}\left|\rho(x)-\rho_{\infty}\right|+\int_{|x|>R_{\epsilon}} \phi_{\left(u_{n}-v_{0}\right)}\left(u_{n}-v_{0}\right)^{2}\left|\rho(x)-\rho_{\infty}\right| \\
& \leq C\left(\|\rho\|_{L^{\infty}}\|\| \nabla \phi_{\left(u_{n}-v_{0}\right)}||_{L^{2}}\left\|u_{n}-v_{0}\right\|_{L^{\frac{12}{5}\left(B_{R_{\epsilon}}\right)}}^{2}+\left.\epsilon\left\|\nabla \phi_{\left(u_{n}-v_{0}\right)}\right\|\right|_{L^{2}}\left\|u_{n}-v_{0}\right\|_{L^{\frac{12}{5}}}^{2}\right),
\end{aligned}
$$

where $C>0$ is a constant. Since $\rho \in L^{\infty}, \phi_{\left(u_{n}-v_{0}\right)}$ is uniformly bounded in $D^{1,2}$ and $u_{n}-v_{0} \rightarrow 0$ in $L_{\text {loc }}^{12 / 5}$, the above shows that $I_{1} \rightarrow 0$ as $n \rightarrow+\infty$. Similarly, we can see that

$$
I_{2} \leq C^{\prime}\left(\|\rho\|_{L^{\infty}}\|\| \nabla \bar{\phi}_{\left(u_{n}-v_{0}\right)}\left\|_{L^{2}}\right\| u_{n}-v_{0}\left\|_{L^{\frac{12}{5}}\left(B_{R_{\epsilon}}\right)}^{2}+\epsilon\right\| \nabla \bar{\phi}_{\left(u_{n}-v_{0}\right)}\left\|_{L^{2}}\right\| u_{n}-v_{0} \|_{L^{\frac{12}{5}}}^{2}\right)
$$

and so $I_{2} \rightarrow 0$ as $n \rightarrow+\infty$. Therefore, we have shown that

$$
\int_{\mathbb{R}^{3}} \rho_{\infty} \bar{\phi}_{\left(u_{n}-v_{0}\right)}\left(u_{n}-v_{0}\right)^{2}=\int_{\mathbb{R}^{3}} \rho \phi_{\left(u_{n}-v_{0}\right)}\left(u_{n}-v_{0}\right)^{2}+o(1)
$$

and thus, by the nonlocal Brezis-Lieb Lemma 2.1, it holds that

$$
\int_{\mathbb{R}^{3}} \rho_{\infty} \bar{\phi}_{\left(u_{n}-v_{0}\right)}\left(u_{n}-v_{0}\right)^{2}=\int_{\mathbb{R}^{3}} \rho \phi_{u_{n}} u_{n}^{2}-\int_{\mathbb{R}^{3}} \rho \phi_{v_{0}} v_{0}^{2}+o(1) .
$$

Putting this together with $(5.10)$, we see that $I_{\mu}^{\infty}\left(u_{n}^{1}\right)=I_{\mu}\left(u_{n}\right)-I_{\mu}\left(v_{0}\right)+o(1)$, and the claim is proved.

Claim 2. $I_{\mu}^{\prime}\left(v_{0}\right)=0$ and $v_{0} \geq 0$.

We notice that for all $\psi \in C_{c}^{\infty}\left(\mathbb{R}^{3}\right)$, it holds that 


$$
I_{\mu}^{\prime}\left(u_{n}\right)(\psi)=\int_{\mathbb{R}^{3}}\left(\nabla u_{n} \nabla \psi+u_{n} \psi\right)+\int_{\mathbb{R}^{3}} \rho \phi_{u_{n}} u_{n} \psi-\mu \int_{\mathbb{R}^{3}}\left(u_{n}\right)_{+}^{p} \psi
$$

Using the fact that $u_{n} \rightarrow v_{0}$ in $H^{1}$ and a local compactness argument, we have $I_{\mu}^{\prime}\left(u_{n}\right)(\psi)=$ $I_{\mu}^{\prime}\left(v_{0}\right)(\psi)+o(1)$. So, since $I_{\mu}^{\prime}\left(u_{n}\right) \rightarrow 0$ by the definition of a Palais-Smale sequence, it holds that $I_{\mu}^{\prime}\left(v_{0}\right)=0$ by density. We note that by testing this equation with $\left(v_{0}\right)_{-}$, we obtain that $v_{0} \geq 0$.

Claim 3. $\left(I_{\mu}^{\infty}\right)^{\prime}\left(u_{n}^{1}\right) \rightarrow 0$.

We first note that by Lemma 5.3, it holds that

$$
\begin{aligned}
\rho \phi_{u_{n}} u_{n} & -\rho_{\infty} \bar{\phi}_{\left(u_{n}-v_{0}\right)}\left(u_{n}-v_{0}\right)-\rho \phi_{v_{0}} v_{0} \\
& =\rho \phi_{u_{n}} u_{n}-\rho \phi_{\left(u_{n}-v_{0}\right)}\left(u_{n}-v_{0}\right)-\rho \phi_{v_{0}} v_{0}+o(1) \\
& =o(1) \quad \text { in } H^{-1}\left(\mathbb{R}^{3}\right) .
\end{aligned}
$$

Moreover, since we have showed in Claim 1 that $\left(u_{n}\right)_{-} \rightarrow 0$ in $L^{p+1}$, then, by Lemma 5.2 , it follows that

$$
\left(u_{n}\right)_{+}^{p}-\left(u_{n}-v_{0}\right)_{+}^{p}-\left(v_{0}\right)^{p}=o(1), \quad \text { in } H^{-1}\left(\mathbb{R}^{3}\right) .
$$

Therefore, using (5.11) and (5.12), we can conclude that

$$
\left(I_{\mu}^{\infty}\right)^{\prime}\left(u_{n}^{1}\right)=I_{\mu}^{\prime}\left(u_{n}\right)-I_{\mu}^{\prime}\left(v_{0}\right)+o(1)
$$

and so

$$
\left(I_{\mu}^{\infty}\right)^{\prime}\left(u_{n}^{1}\right)=o(1)
$$

since $I_{\mu}^{\prime}\left(u_{n}\right) \rightarrow 0$ by the definition of Palais-Smale sequence and $I_{\mu}^{\prime}\left(v_{0}\right)=0$ by Claim 2. This completes the proof of the claim.

Partial conclusions. With these results in place, we now define

$$
\delta:=\limsup _{n \rightarrow+\infty}\left(\sup _{y \in \mathbb{R}^{3}} \int_{B_{1}(y)}\left|u_{n}^{1}\right|^{p+1}\right) .
$$

We can see that $\delta \geq 0$. If $\delta=0$, the P. L. Lions Lemma [36] implies $u_{n}^{1} \rightarrow 0$ in $L^{p+1}$. Since it holds that

$$
\left(I_{\mu}^{\infty}\right)^{\prime}\left(u_{n}^{1}\right)\left(u_{n}^{1}\right)=\left\|u_{n}^{1}\right\|_{H^{1}}^{2}+\int_{\mathbb{R}^{3}} \rho_{\infty} \bar{\phi}_{u_{n}^{1}}\left(u_{n}^{1}\right)^{2}-\mu \int_{\mathbb{R}^{3}}\left(u_{n}^{1}\right)_{+}^{p+1},
$$

and $\left(I_{\mu}^{\infty}\right)^{\prime}\left(u_{n}^{1}\right) \rightarrow 0$ by Claim 3, then, if $u_{n}^{1} \rightarrow 0$ in $L^{p+1}$, it follows that $u_{n}^{1} \rightarrow 0$ in $H^{1}$. In this case, we are done since we have $u_{n} \rightarrow v_{0}$ in $H^{1}$. Therefore, we assume $\delta>0$. This implies that there exists $\left(y_{n}^{1}\right)_{n \in \mathbb{N}} \subset \mathbb{R}^{3}$ such that

$$
\int_{B_{1}\left(y_{n}^{1}\right)}\left|u_{n}^{1}\right|^{p+1}>\frac{\delta}{2} .
$$


We now define $v_{n}^{1}:=u_{n}^{1}\left(\cdot+y_{n}^{1}\right)$. We my assume $v_{n}^{1} \rightarrow v_{1}$ in $H^{1}$ and $v_{n}^{1} \rightarrow v_{1}$ a.e. in $\mathbb{R}^{3}$. Then, since

$$
\int_{B_{1}(0)}\left|v_{n}^{1}\right|^{p+1}>\frac{\delta}{2}
$$

it follows from Rellich Theorem that $v_{1} \not \equiv 0$. Since $u_{n}^{1} \rightarrow 0$ in $H^{1}$, then $\left(y_{n}^{1}\right)_{n \in \mathbb{N}}$ must be unbounded and so we assume, up to a subsequence, $\left|y_{n}^{1}\right| \rightarrow+\infty$. We set $u_{n}^{2}:=u_{n}^{1}-v_{1}\left(\cdot-y_{n}^{1}\right)$, and, using (5.9), we note that

$$
\left\|u_{n}^{2}\right\|_{H^{1}}^{2}=\left\|u_{n}^{1}\right\|_{H^{1}}^{2}-\left\|v_{1}\right\|_{H^{1}}^{2}+o(1)=\left\|u_{n}\right\|_{H^{1}}^{2}-\left\|v_{0}\right\|_{H^{1}}^{2}-\left\|v_{1}\right\|_{H^{1}}^{2}+o(1) .
$$

We now prove three claims regarding the sequence $\left(u_{n}^{2}\right)_{n \in \mathbb{N}}$.

Claim 4. $I_{\mu}^{\infty}\left(u_{n}^{2}\right)=I_{\mu}\left(u_{n}\right)-I_{\mu}\left(v_{0}\right)-I_{\mu}^{\infty}\left(v_{1}\right)+o(1)$.

Arguing similarly as in Claim 1 , namely testing $I_{\mu}^{\infty}\left(u_{n}^{1}\right)$ with $\left(u_{n}^{1}\right)_{-}$, we can show that $\left(u_{n}^{1}\right)_{-} \rightarrow 0$ in $L^{p+1}$, and so $\left(u_{n}^{1}\left(\cdot+y_{n}^{1}\right)\right)_{-} \rightarrow 0$ in $L^{p+1}$. Thus, once again using Lemma 5.1 , we can see that

$$
\begin{aligned}
\left\|\left(u_{n}^{1}\right)_{+}\right\|_{L^{p+1}}^{p+1} & =\left\|\left(u_{n}^{1}\left(\cdot+y_{n}^{1}\right)-v_{1}\right)_{+}\right\|_{L^{p+1}}^{p+1}+\left\|\left(v_{1}\right)_{+}\right\|_{L^{p+1}}^{p+1}+o(1) \\
& =\left\|\left(u_{n}^{1}-v_{1}\left(\cdot-y_{n}^{1}\right)\right)_{+}\right\|_{L^{p+1}}^{p+1}+\left\|\left(v_{1}\right)_{+}\right\|_{L^{p+1}}^{p+1}+o(1) \\
& =\left\|\left(u_{n}^{2}\right)_{+}\right\|_{L^{p+1}}^{p+1}+\left\|\left(v_{1}\right)_{+}\right\|_{L^{p+1}}^{p+1}+o(1),
\end{aligned}
$$

and so

$$
\left\|\left(u_{n}^{2}\right)_{+}\right\|_{L^{p+1}}^{p+1}=\left\|\left(u_{n}^{1}\right)_{+}\right\|_{L^{p+1}}^{p+1}-\left\|\left(v_{1}\right)_{+}\right\|_{L^{p+1}}^{p+1}+o(1) .
$$

Therefore, using this and (5.13), we have that

$$
\begin{gathered}
I_{\mu}^{\infty}\left(u_{n}^{2}\right)=\left\|u_{n}^{1}\right\|_{H^{1}}^{2}-\left\|v_{1}\right\|_{H^{1}}^{2}+\frac{1}{4} \int_{\mathbb{R}^{3}} \rho_{\infty} \bar{\phi}_{\left(u_{n}^{1}-v_{1}\left(-y_{n}^{1}\right)\right)}\left(u_{n}^{1}-v_{1}\left(x-y_{n}^{1}\right)\right)^{2} \\
-\frac{\mu}{p+1}\left(\left\|\left(u_{n}^{1}\right)_{+}\right\|_{L^{p+1}}^{p+1}-\left\|\left(v_{1}\right)_{+}\right\|_{L^{p+1}}^{p+1}\right)+o(1) .
\end{gathered}
$$

We can show, by changing variables and using Lemma 2.1, that

$$
\int_{\mathbb{R}^{3}} \rho_{\infty} \bar{\phi}_{\left(u_{n}^{1}-v_{1}\left(\cdot-y_{n}^{1}\right)\right)}\left(u_{n}^{1}-v_{1}\left(x-y_{n}^{1}\right)\right)^{2}=\int_{\mathbb{R}^{3}} \rho_{\infty} \bar{\phi}_{u_{n}^{1}}\left(u_{n}^{1}\right)^{2}-\int_{\mathbb{R}^{3}} \rho_{\infty} \bar{\phi}_{v_{1}} v_{1}^{2}+o(1)
$$

Thus, by combining the last two equations and using Claim 1 , we see that $I_{\mu}^{\infty}\left(u_{n}^{2}\right)=I_{\mu}^{\infty}\left(u_{n}^{1}\right)-$ $I_{\mu}^{\infty}\left(v_{1}\right)+o(1)=I_{\mu}\left(u_{n}\right)-I_{\mu}\left(v_{0}\right)-I_{\mu}^{\infty}\left(v_{1}\right)+o(1)$, and so the claim is proved.

Claim 5. $\left(I_{\mu}^{\infty}\right)^{\prime}\left(v_{1}\right)=0$ and $v_{1} \geq 0$. 
Let $h \in H^{1}\left(\mathbb{R}^{3}\right)$ and set $h_{n}:=h\left(\cdot-y_{n}^{1}\right)$. By a change of variables, we can see that

$$
\left(I_{\mu}^{\infty}\right)^{\prime}\left(u_{n}^{1}\left(x+y_{n}^{1}\right)\right)(h)=\left(I_{\mu}^{\infty}\right)^{\prime}\left(u_{n}^{1}\right)\left(h_{n}\right)
$$

and so, since $\left(I_{\mu}^{\infty}\right)^{\prime}\left(u_{n}^{1}\right) \rightarrow 0$ by Claim 3 , we have that

$$
\left(I_{\mu}^{\infty}\right)^{\prime}\left(u_{n}^{1}\left(x+y_{n}^{1}\right)\right) \rightarrow 0 .
$$

We now note, for any $\psi \in C_{c}^{\infty}\left(\mathbb{R}^{3}\right)$, it holds that

$$
\begin{gathered}
\left|\int_{\mathbb{R}^{3}} \rho_{\infty} \bar{\phi}_{u_{n}^{1}}\left(x+y_{n}^{1}\right) u_{n}^{1}\left(x+y_{n}^{1}\right) \psi-\int_{\mathbb{R}^{3}} \rho_{\infty} \bar{\phi}_{v_{1}} v_{1} \psi\right| \\
\leq\left|\int_{\mathbb{R}^{3}} \rho_{\infty} \bar{\phi}_{u_{n}^{1}}\left(x+y_{n}^{1}\right)\left(u_{n}^{1}\left(x+y_{n}^{1}\right)-v_{1}\right) \psi\right|+\left|\int_{\mathbb{R}^{3}} \rho_{\infty}\left(\bar{\phi}_{u_{n}^{1}}\left(x+y_{n}^{1}\right)-\bar{\phi}_{v_{1}}\right) v_{1} \psi\right| \\
\leq \rho_{\infty}\left\|\bar{\phi}_{u_{n}^{1}}\left(\cdot+y_{n}^{1}\right)\right\|_{L^{6}}\left\|u_{n}^{1}\left(\cdot+y_{n}^{1}\right)-v_{1}||_{L^{2}(\operatorname{supp} \psi)}\right\| \psi \|_{L^{3}} \\
\quad+\rho_{\infty}\left\|\bar{\phi}_{u_{n}^{1}}\left(\cdot+y_{n}^{1}\right)-\bar{\phi}_{v_{1}}\right\|_{L^{2}(\operatorname{supp} \psi)}\left\|v_{1}\right\|_{L^{6}}\|\psi\|_{L^{3}}
\end{gathered}
$$

and so since $u_{n}^{1}\left(\cdot+y_{n}^{1}\right)-v_{1} \rightarrow 0$ in $L_{\text {loc }}^{2}$ and $\bar{\phi}_{u_{n}^{1}}\left(\cdot+y_{n}^{1}\right)-\bar{\phi}_{v_{1}} \rightarrow 0$ in $L_{\text {loc }}^{2}$, and all of the other terms in the final equation are bounded, then we have shown that

$$
\int_{\mathbb{R}^{3}} \rho_{\infty} \bar{\phi}_{u_{n}^{1}}\left(x+y_{n}^{1}\right) u_{n}^{1}\left(x+y_{n}^{1}\right) \psi \rightarrow \int_{\mathbb{R}^{3}} \rho_{\infty} \bar{\phi}_{v_{1}} v_{1} \psi
$$

Using this and the fact that $u_{n}^{1}\left(\cdot+y_{n}^{1}\right) \rightarrow v_{1}$ in $H^{1}$, it follows by standard arguments that $\left(I_{\mu}^{\infty}\right)^{\prime}\left(u_{n}^{1}\left(x+y_{n}^{1}\right)\right)(\psi)=\left(I_{\mu}^{\infty}\right)^{\prime}\left(v_{1}\right)(\psi)+o(1)$. This implies that $\left(I_{\mu}^{\infty}\right)^{\prime}\left(v_{1}\right)=0$, by (5.14) and density. Testing this equation with $\left(v_{1}\right)_{-}$, shows that $v_{1} \geq 0$.

Claim 6. $\left(I_{\mu}^{\infty}\right)^{\prime}\left(u_{n}^{2}\right) \rightarrow 0$.

We take any $h \in H^{1}\left(\mathbb{R}^{3}\right)$ and set $h_{n}:=h\left(\cdot+y_{n}^{1}\right)$. We note that, by a change of variables, it holds that

$$
\left(I_{\mu}^{\infty}\right)^{\prime}\left(u_{n}^{2}\right)(h)=\left(I_{\mu}^{\infty}\right)^{\prime}\left(u_{n}^{1}\left(\cdot+y_{n}^{1}\right)-v_{1}\right)\left(h_{n}\right)
$$

Now, arguing as we did in the proof of $(i i)$ of Lemma 5.3, we can show that

$$
\rho_{\infty} \bar{\phi}_{u_{n}^{1}}\left(\cdot+y_{n}^{1}\right) u_{n}^{1}\left(\cdot+y_{n}^{1}\right)-\rho_{\infty} \bar{\phi}_{\left(u_{n}^{1}\left(\cdot+y_{n}^{1}\right)-v_{1}\right)}\left(u_{n}^{1}\left(\cdot+y_{n}^{1}\right)-v_{1}\right)-\rho_{\infty} \bar{\phi}_{v_{1}} v_{1}=o(1), \quad \text { in } H^{-1}\left(\mathbb{R}^{3}\right) .
$$

Moreover, since we showed $\left(u_{n}^{1}\left(\cdot+y_{n}^{1}\right)\right)_{-} \rightarrow 0$ in $L^{p+1}$ in Claim 4 , we can once again use Lemma 5.2 to conclude that

$$
\left(u_{n}^{1}\left(\cdot+y_{n}^{1}\right)\right)_{+}^{p}-\left(u_{n}^{1}\left(\cdot+y_{n}^{1}\right)-v_{1}\right)_{+}^{p}-\left(v_{1}\right)_{+}^{p}=o(1), \quad \text { in } H^{-1}\left(\mathbb{R}^{3}\right) .
$$

It follows from this and (5.16) that 


$$
\left(I_{\mu}^{\infty}\right)^{\prime}\left(u_{n}^{1}\left(\cdot+y_{n}^{1}\right)-v_{1}\right)=\left(I_{\mu}^{\infty}\right)^{\prime}\left(u_{n}^{1}\left(\cdot+y_{n}^{1}\right)\right)-\left(I_{\mu}^{\infty}\right)^{\prime}\left(v_{1}\right)+o(1) \quad \text { in } H^{-1}\left(\mathbb{R}^{3}\right) .
$$

Since, by Claim 5 and a change of variables, it holds that

$$
\left(I_{\mu}^{\infty}\right)^{\prime}\left(u_{n}^{1}\left(\cdot+y_{n}^{1}\right)\right)\left(h_{n}\right)-\left(I_{\mu}^{\infty}\right)^{\prime}\left(v_{1}\right)\left(h_{n}\right)=\left(I_{\mu}^{\infty}\right)^{\prime}\left(u_{n}^{1}\right)(h)
$$

then combining this, (5.15) and (5.17), we see that

$$
\left(I_{\mu}^{\infty}\right)^{\prime}\left(u_{n}^{2}\right)=\left(I_{\mu}^{\infty}\right)^{\prime}\left(u_{n}^{1}\right)+o(1) \quad \text { in } H^{-1}\left(\mathbb{R}^{3}\right)
$$

It therefore follows that $\left(I_{\mu}^{\infty}\right)^{\prime}\left(u_{n}^{2}\right) \rightarrow 0$ since $\left(I_{\mu}^{\infty}\right)^{\prime}\left(u_{n}^{1}\right) \rightarrow 0$ by Claim 3, and we are done.

Conclusions. With these results in place we can now see that if $u_{n}^{2} \rightarrow 0$ in $H^{1}$, then we are done. Otherwise, $u_{n}^{2} \rightarrow 0$ in $H^{1}$, but not strongly, and so we repeat the argument. By iterating the procedure, we obtain sequences of points $\left(y_{n}^{j}\right)_{n \in \mathbb{N}} \subset \mathbb{R}^{3}$ such that $\left|y_{n}^{j}\right| \rightarrow+\infty$, $\left|y_{n}^{j}-y_{n}^{j^{\prime}}\right| \rightarrow+\infty$ as $n \rightarrow+\infty$ if $j \neq j^{\prime}$ and a sequence of functions $\left(u_{n}^{j}\right)_{n \in \mathbb{N}}$ with $u_{n}^{1}=u_{n}-v_{0}$ and $u_{n}^{j}=u_{n}^{j-1}-v_{j-1}\left(\cdot-y_{n}^{j-1}\right)$ for $j \geq 2$ such that

$$
\begin{aligned}
& u_{n}^{j}\left(x+y_{n}^{j}\right) \rightarrow v_{j}(x) \text { in } H^{1}, \\
& \left\|u_{n}\right\|_{H^{1}\left(\mathbb{R}^{3}\right)}^{2}=\sum_{j=0}^{l-1}\left\|v_{j}\right\|_{H^{1}\left(\mathbb{R}^{3}\right)}^{2}+\left\|u_{n}^{l}\right\|_{H^{1}}^{2}+o(1), \\
& \left\|u_{n}-v_{0}-\sum_{j=1}^{l} v_{j}\left(\cdot-y_{n}^{j}\right)\right\|_{H^{1}\left(\mathbb{R}^{3}\right)} \rightarrow 0 \text { as } n \rightarrow+\infty, \\
& I_{\mu}\left(u_{n}\right)=I_{\mu}\left(v_{0}\right)+\sum_{j=1}^{l-1} I_{\mu}^{\infty}\left(v_{j}\right)+I_{\mu}^{\infty}\left(u_{n}^{l}\right)+o(1), \\
& \left(I_{\mu}^{\infty}\right)^{\prime}\left(v_{j}\right)=0 \text { and } v_{j} \geq 0 \text { for } j \geq 1,
\end{aligned}
$$

We notice from the last equation that it holds that $\left(I_{\mu}^{\infty}\right)^{\prime}\left(v_{j}\right)\left(v_{j}\right)=0$ for each $j \geq 1$. Using this, the Sobolev embedding theorem and the fact that $\mu \leq 1$, we have that

$$
S_{p+1}\left\|v_{j}\right\|_{L^{p+1}}^{2} \leq\left\|v_{j}\right\|_{H^{1}}^{2} \leq\left\|v_{j}\right\|_{H^{1}}^{2}+\int_{\mathbb{R}^{3}} \rho_{\infty} \bar{\phi}_{v_{j}}\left(v_{j}\right)^{2}=\mu\left\|\left(v_{j}\right)_{+}\right\|_{L^{p+1}}^{p+1} \leq\left\|v_{j}\right\|_{L^{p+1}}^{p+1},
$$

and therefore, we can conclude that, for each $j \geq 1$,

$$
\left\|v_{j}\right\|_{H^{1}}^{2} \geq\left(S_{p+1}\right)^{\frac{p+1}{p-1}}
$$

Combining this and the fact $\left(u_{n}\right)_{n \in \mathbb{N}}$ is bounded in $H^{1}$, we see from (5.18) that the iteration must stop at some finite index $l \in \mathbb{N}$. 
5.2. Proof of Theorem 3. We are finally in position to establish two sufficient conditions that guarantee the existence of a mountain pass solution to (1.1) in the case of non-coercive $\rho$, if $p \in(2,3)$.

Proof of Theorem 3. We first note that by Lemma 3.2 with $E=H^{1}$, the set $\mathcal{M}$, defined in (3.6), is nonempty.

Claim 1. Under assumptions $(i)$, the values $c_{\mu}$ are critical levels of $I_{\mu}$ for all $\mu \in(1-\epsilon, 1] \cap \mathcal{M}$, with $\epsilon>0$ sufficiently small. Namely, there exists a nonnegative $u \in H^{1}$ such that $I_{\mu}(u)=c_{\mu}$ and $I_{\mu}^{\prime}(u)=0$ for all $\mu \in(1-\epsilon, 1] \cap \mathcal{M}$. Under assumptions (ii), the same statement holds for all $\mu \in \mathcal{M}$.

We recall that for all $\mu \in \mathcal{M}$, by definition, there exists a bounded sequence $\left(u_{n}\right)_{n \in \mathbb{N}} \subset H^{1}$ such that $I_{\mu}\left(u_{n}\right) \rightarrow c_{\mu}$ and $I_{\mu}^{\prime}\left(u_{n}\right) \rightarrow 0$. We note that by Proposition 2 and the definition of $\left(u_{n}\right)_{n \in \mathbb{N}}$, it holds that

$$
c_{\mu}=I_{\mu}\left(v_{0}\right)+\sum_{j=1}^{l} I_{\mu}^{\infty}\left(v_{j}\right),
$$

where $v_{0}$ is a nonnegative solution of (3.1) and $v_{j}$ are nonnegative solutions of (3.8) for $1 \leq j \leq l$.

Assume that $(i)$ holds. For $\epsilon>0$ small enough, it holds that $c_{\mu}<c_{\mu}^{\infty}$ for all $\mu \in(1-\epsilon, 1] \cap \mathcal{M}$, by continuity. Pick $\mu$ on this set. If $v_{j}$ is nontrivial for some $1 \leq j \leq l$, it would follow that $I_{\mu}^{\infty}\left(v_{j}\right) \geq c_{\mu}^{\infty}>c_{\mu}$ by Lemma 3.4. This is in contradiction with (5.19) since $I_{\mu}\left(v_{0}\right) \geq 0$, by Lemma 3.3, and so, $v_{j} \equiv 0$ for all $1 \leq j \leq l$. Therefore, $u_{n} \rightarrow v_{0}$ in $H^{1}$ by $(i v)$ of Proposition 2 , $I_{\mu}\left(v_{0}\right)=c_{\mu}$ by (5.19), and $I_{\mu}^{\prime}\left(v_{0}\right)=0$ since $v_{0}$ is a nonnegative solution of (3.1). Thus, we have shown $c_{\mu}$ is a critical level of $I_{\mu}$ in this case.

Now, assume that (ii) holds. We note that this implies that $I_{\mu}(\gamma(t)) \leq I_{\mu}^{\infty}(\gamma(t))$ for each fixed $\gamma \in \Gamma^{\infty}, \mu \in\left[\frac{1}{2}, 1\right]$ and $t \in[0,1]$. It therefore follows that $I_{\frac{1}{2}}(\gamma(1)) \leq I_{\frac{1}{2}}^{\infty}(\gamma(1))<0$ for all $\gamma \in \Gamma^{\infty}$, and so $\Gamma^{\infty} \subseteq \Gamma$. Using this and Lemma 3.4, we can see that for each nontrivial $v_{j}$ in (5.19), it holds

$$
\begin{aligned}
I_{\mu}^{\infty}\left(v_{j}\right) & \geq c_{\mu}^{\infty} \\
& =\inf _{\gamma \in \Gamma^{\infty}} \max _{t \in[0,1]} I_{\mu}^{\infty}(\gamma(t)), \\
& \geq \inf _{\gamma \in \Gamma^{\infty}} \max _{t \in[0,1]} I_{\mu}(\gamma(t)) \\
& \geq \inf _{\gamma \in \Gamma} \max _{t \in[0,1]} I_{\mu}(\gamma(t)) \\
& =c_{\mu} .
\end{aligned}
$$

We now assume, by contradiction, $v_{0} \equiv 0$ in (5.19), which would imply $I_{\mu}\left(v_{0}\right)=0$. Using this and (5.20), we see from (5.19) that there exists one nontrivial $v_{j}$, call it $v_{1}$, such that $v_{1}$ is a nonnegative solution of (3.8) and 


$$
I_{\mu}^{\infty}\left(v_{1}\right)=c_{\mu}^{\infty}=c_{\mu} .
$$

Define $\bar{v}_{t}(x)=t^{2} v_{1}(t x)$ and $\gamma: \mathbb{R} \rightarrow H^{1}\left(\mathbb{R}^{3}\right), \gamma(t)=\bar{v}_{t}$. By Lemma 3.3 in [45, p. 663], the function $f(t)=I_{\mu}^{\infty}\left(\bar{v}_{t}\right)$ has a unique critical point corresponding to its maximum, and it can be shown that $f^{\prime}(1)=0$ by Nehari's and Pohozaev's identities for $v_{1}$. We deduce that

$$
\max _{t \in \mathbb{R}} I_{\mu}^{\infty}(\gamma(t))=I_{\mu}^{\infty}\left(v_{1}\right)
$$

and that there exists $M>0$ such that

$$
I_{\frac{1}{2}}^{\infty}(\gamma(M))<0
$$

and

$$
\max _{t \in \mathbb{R}} I_{\mu}^{\infty}(\gamma(t))=\max _{t \in[0, M]} I_{\mu}^{\infty}(\gamma(t)) .
$$

We then define $\gamma_{0}:[0,1] \rightarrow H^{1}\left(\mathbb{R}^{3}\right), \gamma_{0}(t)=\gamma(M t)$, and see from the above work that $\gamma_{0} \in \Gamma^{\infty}$. Therefore, we have that

$$
\begin{aligned}
I_{\mu}^{\infty}\left(v_{1}\right) & =\max _{t \in \mathbb{R}} I_{\mu}^{\infty}(\gamma(t)) \\
& =\max _{t \in[0, M]} I_{\mu}^{\infty}(\gamma(t)) \\
& =\max _{t \in[0,1]} I_{\mu}^{\infty}\left(\gamma_{0}(t)\right) .
\end{aligned}
$$

Since we have $v_{1}>0$ on $B$ where $\rho(x)<\rho_{\infty}$ by Lemma 3.4, it follows that

$$
\begin{aligned}
c_{\mu}^{\infty} & =I_{\mu}^{\infty}\left(v_{1}\right) \\
& =\max _{t \in[0,1]} I_{\mu}^{\infty}\left(\gamma_{0}(t)\right) \\
& >\max _{t \in[0,1]} I_{\mu}\left(\gamma_{0}(t)\right) \\
& \geq \inf _{\gamma \in \Gamma \infty} \max _{t \in[0,1]} I_{\mu}(\gamma(t)) \\
& \geq \inf _{\gamma \in \Gamma} \max _{t \in[0,1]} I_{\mu}(\gamma(t)) \\
& =c_{\mu},
\end{aligned}
$$

which contradicts (5.21). Therefore, we have shown that $v_{0} \not \equiv 0$. Now, since $v_{0}$ is a nontrivial and nonnegative solution of (3.1), then $I_{\mu}\left(v_{0}\right)>0$ by Lemma 3.3. Putting this and (5.20) together in (5.19), it follows that $v_{j} \equiv 0$ for all $1 \leq j \leq l$. Therefore, $u_{n} \rightarrow v_{0}$ in $H^{1}$ by $(i v)$ of Proposition 2 , $I_{\mu}\left(v_{0}\right)=c_{\mu}$ by $(5.19)$, and $I_{\mu}^{\prime}\left(v_{0}\right)=0$ since $v_{0}$ is a nonnegative solution of (3.1). This concludes the proof of Claim 1.

Claim 2. Let $\mu_{n} \rightarrow 1$ be an increasing sequence in $(1-\epsilon, 1] \cap \mathcal{M}$ and (resp.) $\mathcal{M}$ under assumptions (i) and (resp.) (ii). Assume $\left(u_{n}\right)_{n \in \mathbb{N}} \subset H^{1}$ is such that $u_{n}$ is nonnegative, $I_{\mu_{n}}\left(u_{n}\right)=c_{\mu_{n}}$ and 
$I_{\mu_{n}}^{\prime}\left(u_{n}\right)=0$ for each $n$. Then, there exists a nonnegative $u \in H^{1}$ such that, up to a subsequence, $u_{n} \rightarrow u$ in $H^{1}, I(u)=c$, and $I^{\prime}(u)=0$.

Since $u_{n}$ is nonnegative, $\left(u_{n}\right)_{+}=u_{n}$, and so we can see that

$$
\begin{aligned}
I\left(u_{n}\right) & =I_{\mu_{n}}\left(u_{n}\right)+\frac{\mu_{n}-1}{p+1} \int_{\mathbb{R}^{3}} u_{n}^{p+1} \\
& =c_{\mu_{n}}+\frac{\mu_{n}-1}{p+1} \int_{\mathbb{R}^{3}} u_{n}^{p+1},
\end{aligned}
$$

and, for all $v \in H^{1}\left(\mathbb{R}^{3}\right)$,

$$
\begin{aligned}
I^{\prime}\left(u_{n}\right)(v) & =I_{\mu_{n}}^{\prime}\left(u_{n}\right)(v)+\left(\mu_{n}-1\right) \int_{\mathbb{R}^{3}} u_{n}^{p} v \\
& \leq\left|\mu_{n}-1\right|\left\|u_{n}||_{L^{p+1}}^{p}\right\| v \|_{L^{p+1}} \\
& \leq S_{p+1}^{-\frac{1}{2}}\left|\mu_{n}-1\right|\left\|u_{n}\right\|_{L^{p+1}}^{p}\|v\|_{H^{1}} .
\end{aligned}
$$

Set $\alpha_{n}=\int_{\mathbb{R}^{3}}\left(\left|\nabla u_{n}\right|^{2}+u_{n}^{2}\right), \gamma_{n}=\int_{\mathbb{R}^{3}} \rho \phi_{u_{n}} u_{n}^{2}$, and $\delta_{n}=\mu_{n} \int_{\mathbb{R}^{3}} u_{n}^{p+1}$. As in Theorem 2 Claim 2, we see that $\alpha_{n}, \gamma_{n}$, and $\delta_{n}$ satisfy (4.11), and thus we can obtain that $\alpha_{n}, \gamma_{n}$, and $\delta_{n}$ are all bounded. Therefore, using this, (5.22), (5.23), and the fact that $c_{\mu_{n}} \rightarrow c$ as $\mu_{n} \nearrow 1$ by definition (3.7), we can deduce that $\left\|u_{n}\right\|_{H^{1}}$ is bounded, $I\left(u_{n}\right) \rightarrow c$ and $I^{\prime}\left(u_{n}\right) \rightarrow 0$ as $n \rightarrow+\infty$. That is, we have shown that $\left(u_{n}\right)_{n \in \mathbb{N}}$ is a bounded Palais-Smale sequence for $I=I_{1}$ at the level $c=c_{1}$, and so, $1 \in \mathcal{M}$. By Claim 1, it follows that there exists a nonnegative $u \in H^{1}$ such that, up to a subsequence, $u_{n} \rightarrow u$ in $H^{1}, I(u)=c$, and $I^{\prime}(u)=0$.

Conclusion. Let $\mu_{n} \rightarrow 1$ be an increasing sequence in $(1-\epsilon, 1] \cap \mathcal{M}$ and (resp.) $\mathcal{M}$ under assumptions $(i)$ and (resp.) (ii). By Claim 1, we can choose $\left(u_{n}\right)_{n \in \mathbb{N}} \subset H^{1}$ such that $u_{n}$ is nonnegative, $I_{\mu_{n}}\left(u_{n}\right)=c_{\mu_{n}}$ and $I_{\mu_{n}}^{\prime}\left(u_{n}\right)=0$ for each $n$. By Claim 2, it follows that there exists a nonnegative $u \in H^{1}$ such that, up to a subsequence, $u_{n} \rightarrow u$ in $H^{1}, I(u)=c$, and $I^{\prime}(u)=0$. That is, we have shown $\left(u, \phi_{u}\right) \in H^{1}\left(\mathbb{R}^{3}\right) \times D^{1,2}\left(\mathbb{R}^{3}\right)$ solves (1.1). Since $u$ and $\phi_{u}$ are nonnegative by construction, by regularity and the strong maximum principle, it follows that they are, in fact, strictly positive. This concludes the proof.

\subsection{Proof of Theorem 4.}

Proposition 4. [Palais-Smale condition for $p \geq 3$ ] Let $\rho \in C\left(\mathbb{R}^{3}\right)$ be nonnegative such that $\rho(x) \rightarrow \rho_{\infty}>0$ as $|x| \rightarrow+\infty$ and suppose either of the following conditions hold:

(i) $c<c^{\infty}$

or

(ii) $\rho(x) \leq \rho_{\infty}$ for all $x \in \mathbb{R}^{3}$, with strict inequality, $\rho(x)<\rho_{\infty}$, on some ball $B \subset \mathbb{R}^{3}$,

where $c$ and (resp.) $c^{\infty}$ are defined in (3.7) and (resp.) (3.11). Then, for any $p \in[3,5)$, every Palais-Smale sequence $\left(u_{n}\right)_{n \in \mathbb{N}} \subset H^{1}\left(\mathbb{R}^{3}\right)$ for $I$, at the level $c$, is relatively compact. In particular, $c$ is a critical level for $I$. 
Proof. Since, for $p \geq 3$, we have

$$
c+1+o(1)\left\|u_{n}\right\|_{H^{1}\left(\mathbb{R}^{3}\right)} \geq(p+1) I\left(u_{n}\right)-I^{\prime}\left(u_{n}\right) u_{n} \geq\left\|u_{n}\right\|_{H^{1}\left(\mathbb{R}^{3}\right)}^{2},
$$

it follows that $\left(u_{n}\right)_{n \in \mathbb{N}}$ is bounded. By the definition of $u_{n}$ and Proposition 2 with $\mu=1$, it holds that

$$
c=I\left(v_{0}\right)+\sum_{j=1}^{l} I^{\infty}\left(v_{j}\right)
$$

where $v_{0}$ is a nonnegative solution of (1.9) and $v_{j}$ are nonnegative solutions of (1.13) for $1 \leq j \leq l$. Reasoning as in Claim 1 of Theorem 3, setting $\mu=1$ and replacing $c_{\mu}, c_{\mu}^{\infty}, \Gamma$, and $\Gamma^{\infty}$ with $c, c^{\infty}$, $\bar{\Gamma}$, and $\bar{\Gamma}^{\infty}$, respectively, throughout, the statement follows. This concludes the proof.

Proof of Theorem 4. The regularity and the strong maximum principle imply that the nontrivial and nonnegative critical point, $u$, of $I$, found in Proposition 4, is strictly positive. For the same reason, $\phi_{u}>0$ everywhere.

\subsection{Proof of Corollary 2.}

Proof of Corollary 2. If $p \in(3,5)$, we can use the Nehari characterisation of the mountain pass level (4.17) with $E=H^{1}$ to see that the mountain pass solution $u$ found in Theorem 4 is a least energy solution for $I$. If $p \in(2,3]$, we set

$$
c^{*}:=\inf _{u \in \mathcal{A}} I(u), \text { where } \mathcal{A}:=\left\{u \in H^{1}\left(\mathbb{R}^{3}\right) \backslash\{0\}: u \text { is a nonnegative solution to }(1.9)\right\}
$$

and can show that $\mathcal{A}$ is nonempty and $c^{*}$ is well-defined using the mountain pass critical points that we found in Theorem 4 and Theorem 3 when $p=3$ and $p \in(2,3)$, respectively. It is important to note that when $p=3$, the critical point, $u \in \mathcal{A}$, that we found in Theorem 4 satisfies $I(u)=c$, which implies $c^{*} \leq c$. Similarly, when $p \in(2,3)$, we can show $c^{*} \leq c$ using the critical point that we found in Theorem 3. Now, for any $p \in(2,3)$, arguing as in the proof of Corollary 1 , we can show that there exists a bounded sequence $\left(w_{n}\right)_{n \in \mathbb{N}} \subset \mathcal{A}$ such that $I\left(w_{n}\right) \rightarrow c^{*}$ as $n \rightarrow+\infty$ and $I^{\prime}\left(w_{n}\right)=0$. By applying Proposition 2 with $\mu=1$ to $\left(w_{n}\right)_{n \in \mathbb{N}}$, we can see that

$$
c \geq c^{*}=I\left(v_{0}\right)+\sum_{j=1}^{l} I^{\infty}\left(v_{j}\right),
$$

where $v_{0}$ is a nonnegative solution of (1.9) and $v_{j}$ are nonnegative solutions of $(1.13)$ for $1 \leq j \leq l$. Reasoning as in Claim 1 of Theorem 3 with $\mu=1$ and replacing $c_{\mu}, c_{\mu}^{\infty}, \Gamma$, and $\Gamma^{\infty}$ with $c, c^{\infty}$, $\bar{\Gamma}$, and $\bar{\Gamma}^{\infty}$, respectively, throughout, we can show $I\left(v_{0}\right)=c^{*}$ and $I^{\prime}\left(v_{0}\right)=0$. We note that by Lemma 3.3, it holds that $c^{*} \geq C>0$ for some uniform constant $C>0$, and so it follows that $v_{0}$ is a nontrivial least energy critical point of $I$. The strict positivity of $v_{0}$ and $\phi_{v_{0}}$ follows by regularity and the strong maximum principle since they are nonnegative by construction. This concludes the proof. 


\section{NECESSARY CONDITIONS FOR CONCENTRATION OF SEMIClASSiCAL STATES}

6.1. Proof of Theorem 5. We first prove a necessary condition for the concentration of positive solutions in the semiclassical limit, $\epsilon \rightarrow 0^{+}$, in $E$.

Proof of Theorem 5. We will break the proof into five claims.

Claim 1. $\sup _{\epsilon>0}|| u_{\epsilon} \|_{L^{\infty}\left(\mathbb{R}^{3}\right)}<+\infty$

We will argue by contradiction. Assume, to the contrary, that there exists a sequence $\left(\epsilon_{m}\right)_{m \in \mathbb{N}}$ such that $\epsilon_{m} \rightarrow 0$ as $m \rightarrow+\infty, u_{m}:=u_{\epsilon_{m}}$ solves (1.15) for each $m$, and it holds

$$
\left\|u_{m}\right\|_{L^{\infty}\left(\mathbb{R}^{3}\right)} \rightarrow+\infty \text { as } m \rightarrow+\infty .
$$

Let

$$
\begin{gathered}
\alpha_{m}:=\max u_{m}, \quad\left(\alpha_{m} \rightarrow+\infty \text { as } m \rightarrow+\infty\right), \\
\beta_{m}:=\alpha_{m}^{-(p-1) / 2}, \quad\left(\beta_{m} \rightarrow 0 \text { as } m \rightarrow+\infty\right) .
\end{gathered}
$$

Define

$$
v_{m}(x):=\frac{1}{\alpha_{m}} u_{m}\left(x_{m}+\epsilon_{m} \beta_{m} x\right)
$$

where $x_{m}$ is a global maximum point of $u_{m}$. We note that such a point exists because, by regularity theory, $u_{m}$ are solutions in the classical sense and, moreover, by the concentration assumption, $u_{m}$ decays to zero uniformly with respect to $m$. Now, multiplying (1.15) by $\frac{\beta_{m}^{2}}{\alpha_{m}}$, we obtain

$$
\begin{aligned}
& -\frac{\epsilon_{m}^{2} \beta_{m}^{2}}{\alpha_{m}} \Delta u_{m}\left(x_{m}+\epsilon_{m} \beta_{m} x\right)+\frac{\beta_{m}^{2}}{\alpha_{m}} \lambda u_{m}\left(x_{m}+\epsilon_{m} \beta_{m} x\right) \\
& \quad+\beta_{m}^{2} \rho\left(x_{m}+\epsilon_{m} \beta_{m} x\right) \phi_{u_{m}}\left(x_{m}+\epsilon_{m} \beta_{m} x\right) \frac{1}{\alpha_{m}} u_{m}\left(x_{m}+\epsilon_{m} \beta_{m} x\right)=\frac{\beta_{m}^{2}}{\alpha_{m}} u_{m}^{p}\left(x_{m}+\epsilon_{m} \beta_{m} x\right) .
\end{aligned}
$$

Noting that $\Delta v_{m}(x)=\epsilon_{m}^{2} \beta_{m}^{2} \Delta u_{m}\left(x_{m}+\epsilon_{m} \beta_{m} x\right) / \alpha_{m}$ and $\beta_{m}^{2} / \alpha_{m}=1 / \alpha_{m}^{p}$, we see that $v_{m}$ satisfies

$$
-\Delta v_{m}+\beta_{m}^{2} \lambda v_{m}+\beta_{m}^{2} \rho\left(x_{m}+\epsilon_{m} \beta_{m} x\right) \phi_{u_{m}}\left(x_{m}+\epsilon_{m} \beta_{m} x\right) v_{m}=v_{m}^{p} .
$$

We further note that

$$
\begin{aligned}
\phi_{u_{m}}\left(x_{m}+\epsilon_{m} \beta_{m} x\right) & =\int_{\mathbb{R}^{3}} \frac{u_{m}^{2}(y) \rho(y)}{4 \pi\left|x_{m}+\epsilon_{m} \beta_{m} x-y\right|} \mathrm{d} y \\
& =\int_{\mathbb{R}^{3}} \frac{u_{m}^{2}\left(x_{m}+\epsilon_{m} \beta_{m} y\right) \rho\left(x_{m}+\epsilon_{m} \beta_{m} y\right)}{4 \pi\left|x_{m}+\epsilon_{m} \beta_{m} x-x_{m}-\epsilon_{m} \beta_{m} y\right|} \cdot \epsilon_{m}^{3} \beta_{m}^{3} \mathrm{~d} y \\
& =\epsilon_{m}^{2} \beta_{m}^{2} \alpha_{m}^{2} \int_{\mathbb{R}^{3}} \frac{v_{m}^{2}(y) \rho\left(x_{m}+\epsilon_{m} \beta_{m} y\right)}{4 \pi|x-y|} \mathrm{d} y
\end{aligned}
$$


where we have used the change of variables $y \rightarrow x_{m}+\epsilon_{m} \beta_{m} y$ in going from the first to second line. Therefore, $v_{m}$ satisfies

$$
-\Delta v_{m}+\beta_{m}^{2} \lambda v_{m}+\beta_{m}^{2} \rho\left(x_{m}+\epsilon_{m} \beta_{m} x\right)\left(\epsilon_{m}^{2} \beta_{m}^{2} \alpha_{m}^{2} \int_{\mathbb{R}^{3}} \frac{v_{m}^{2}(y) \rho\left(x_{m}+\epsilon_{m} \beta_{m} y\right)}{4 \pi|x-y|} \mathrm{d} y\right) v_{m}=v_{m}^{p} .
$$

Namely, since $\beta_{m}^{4} \alpha_{m}^{2}=\alpha_{m}^{-2(p-1)} \alpha_{m}^{2}=\alpha_{m}^{4-2 p}$ (by the definition of $\beta$ ), we have that $v_{m}$ satisfies

$$
-\Delta v_{m}=-\beta_{m}^{2} \lambda v_{m}-\epsilon_{m}^{2} \alpha_{m}^{4-2 p} \rho\left(x_{m}+\epsilon_{m} \beta_{m} x\right)\left(\int_{\mathbb{R}^{3}} \frac{v_{m}^{2}(y) \rho\left(x_{m}+\epsilon_{m} \beta_{m} y\right)}{4 \pi|x-y|} \mathrm{d} y\right) v_{m}+v_{m}^{p}
$$

It is worth noting here that since $\alpha_{m} \rightarrow+\infty$ as $m \rightarrow+\infty$, then $\alpha_{m}^{4-2 p} \rightarrow 0$ as $m \rightarrow+\infty$ for $p>2$ and $\alpha_{m}^{4-2 p} \rightarrow 1$ as $m \rightarrow+\infty$ for $p=2^{2}$.

We now fix some compact set $K$. We notice that, by construction, $\left\|v_{m}\right\|_{L^{\infty}\left(\mathbb{R}^{3}\right)}=1$ for all $m$, and, by assumption, $\rho$ is continuous. We also highlight that due to the concentration assumption, we have that the sequence of global maximum points $x_{m}$ is uniformly bounded with respect to $\mathrm{m}$. So, since $v_{m}^{2} \rho$ is uniformly bounded in $L^{\infty}(K)$, then $\int_{\mathbb{R}^{3}} \frac{v_{m}^{2}(y) \rho\left(x_{m}+\epsilon_{m} \beta_{m} y\right)}{4 \pi|x-y|} \mathrm{d} y$ is uniformly bounded in $C^{0, \alpha}(K)$ and consequently, is uniformly bounded in $L^{\infty}(K)$ (see e.g. [34, p. 260]; [1, p. 11]). Thus, the entire right-hand side of (6.1) is uniformly bounded in $L^{\infty}(K)$ which implies $v_{m}$ is uniformly bounded in $C^{1, \alpha}(K)$ (see e.g. [27]). It then follows that the right-hand side of (6.1) is uniformly bounded in $C^{0, \alpha}(K)$, and therefore $v_{m}$ is uniformly bounded in $C^{2, \alpha}(K)$ by Schauder estimates (see e.g. [27]). Namely, for $x, y \in K, x \neq y$, and for every $m$, it holds that

$$
\left|\partial^{\beta} v_{m}(x)-\partial^{\beta} v_{m}(y)\right| \leq C_{K}|x-y|^{\alpha}, \quad|\beta| \leq 2
$$

for some constant $C_{K}$ which depends on $K$ but does not depend on $m$. It follows that uniformly on compact sets and for some $v_{0} \in C^{2}\left(\mathbb{R}^{3}\right)$,

$$
\partial^{\beta} v_{m} \rightarrow \partial^{\beta} v_{0} \text { as } m \rightarrow+\infty, \quad|\beta| \leq 2
$$

Therefore, taking the limit $m \rightarrow+\infty$ in (6.1) we get

$$
\left\{\begin{aligned}
-\Delta v_{0} & =v_{0}^{p}, \quad x \in \mathbb{R}^{3} \\
v_{0}(0) & =1,
\end{aligned}\right.
$$

where the second equality has come from the fact that $v_{m}(0)=u_{m}\left(x_{m}\right) / \alpha_{m}=\alpha_{m} / \alpha_{m}=1$ for all $m$. On the other hand from the equation, by a celebrated result of Gidas-Spruck [26] we infer $v_{0} \equiv 0$. So, we have reached a contradiction, and thus $\sup _{\epsilon>0}\left\|u_{\epsilon}\right\|_{L^{\infty}\left(\mathbb{R}^{3}\right)}<+\infty$.

Claim 2. Assume there exists a sequence $\left(\epsilon_{k}\right)_{k \in \mathbb{N}}$ such that $\epsilon_{k} \rightarrow 0$ as $k \rightarrow+\infty$ and $u_{k}:=u_{\epsilon_{k}}$ solves (1.15) for each $k$. Let $w_{k}(x):=u_{k}\left(x_{0}+\epsilon_{k} x\right)$, where $x_{0}$ is a concentration point for $u_{k}$. Then,

(i) up to a subsequence, $w_{k} \rightarrow$ some $w_{0}$ in $C_{\text {loc }}^{2}\left(\mathbb{R}^{3}\right)$,

\footnotetext{
${ }^{2}$ This is the only point in which we use the restriction $p \geq 2$.
} 
(ii) $w_{0}>0$.

We begin by proving $(i)$. We first notice that $w_{k}$ solves

$$
\begin{cases}-\Delta w_{k}+\lambda w_{k}+\rho\left(x_{0}+\epsilon_{k} x\right) \phi_{u_{k}}\left(x_{0}+\epsilon_{k} x\right) w_{k}=w_{k}^{p}, & x \in \mathbb{R}^{3} \\ -\Delta \phi_{u_{k}}\left(x_{0}+\epsilon_{k} x\right)=\rho\left(x_{0}+\epsilon_{k} x\right) w_{k}^{2}, & x \in \mathbb{R}^{3}\end{cases}
$$

We note that

$$
\begin{aligned}
\phi_{u_{k}}\left(x_{0}+\epsilon_{k} x\right) & =\int_{\mathbb{R}^{3}} \frac{u_{k}^{2}(y) \rho(y)}{4 \pi\left|x_{0}+\epsilon_{k} x-y\right|} \mathrm{d} y \\
& =\int_{\mathbb{R}^{3}} \frac{u_{k}^{2}\left(x_{0}+\epsilon_{k} y\right) \rho\left(x_{0}+\epsilon_{k} y\right)}{4 \pi\left|x_{0}+\epsilon_{k} x-x_{0}-\epsilon_{k} y\right|} \cdot \epsilon_{k}^{3} \mathrm{~d} y \\
& =\epsilon_{k}^{2} \int_{\mathbb{R}^{3}} \frac{w_{k}^{2}(y) \rho\left(x_{0}+\epsilon_{k} y\right)}{4 \pi|x-y|} \mathrm{d} y,
\end{aligned}
$$

where we have used the change of variables $y \rightarrow x_{0}+\epsilon_{k} y$ in going from the first to second line. So, $w_{k}$ solves

$$
-\Delta w_{k}=-\lambda w_{k}-\rho\left(x_{0}+\epsilon_{k} x\right)\left(\epsilon_{k}^{2} \int_{\mathbb{R}^{3}} \frac{w_{k}^{2}(y) \rho\left(x_{0}+\epsilon_{k} y\right)}{4 \pi|x-y|} \mathrm{d} y\right) w_{k}+w_{k}^{p} .
$$

We now once again fix some compact set $K$. We notice that, by Claim $1, \sup _{k>0}\left\|w_{k}\right\|_{L^{\infty}\left(\mathbb{R}^{3}\right)}<$ $+\infty$, and, by assumption, $\rho$ is continuous. So, since $w_{k}^{2} \rho$ is uniformly bounded in $L^{\infty}(K)$, then $\int_{\mathbb{R}^{3}} \frac{w_{k}^{2}(y) \rho\left(x_{0}+\epsilon_{k} y\right)}{4 \pi|x-y|} \mathrm{d} y$ is uniformly bounded in $C^{0, \alpha}(K)$ and thus, is uniformly bounded in $L^{\infty}(K)$ (see e.g. [34, p. 260]; [1, p. 11]). Therefore, the right-hand side of (6.3) is uniformly bounded in $L^{\infty}(K)$ which implies $w_{k}$ is uniformly bounded in $C^{1, \alpha}(K)$ (see e.g. [27]). It follows that the right-hand side of (6.3) is uniformly bounded in $C^{0, \alpha}(K)$, and thus, by Schauder estimates, we have that $w_{k}$ is uniformly bounded in $C^{2, \alpha}(K)$ (see e.g. [27]). Since this holds for every compact set contained in $\mathbb{R}^{3}$, arguing the same way as in Claim 1 , it follows that uniformly on compact sets and for some $w_{0} \in C^{2}\left(\mathbb{R}^{3}\right)$,

$$
\partial^{\beta} w_{k} \rightarrow \partial^{\beta} w_{0} \text { as } k \rightarrow+\infty, \quad|\beta| \leq 2 .
$$

Therefore, taking the limit $k \rightarrow+\infty$ in (6.3), we have

$$
-\Delta w_{0}+\lambda w_{0}=w_{0}^{p}, \quad x \in \mathbb{R}^{3} .
$$

We now aim to prove $(i i)$. Let $x_{k}$ be a maximum point of $u_{k}$. Since $u_{k}$ is a solution to (1.15), we have that

$$
-\epsilon_{k}^{2} \Delta u_{k}\left(x_{k}\right)+\lambda u_{k}\left(x_{k}\right)+\rho\left(x_{k}\right) \phi_{u_{x_{k}}}\left(x_{k}\right) u_{k}\left(x_{k}\right)=u_{k}^{p}\left(x_{k}\right)
$$

Noting that $\Delta u_{k}\left(x_{k}\right) \leq 0$ since $x_{k}$ is a maximum point of $u_{k}$, we see that 


$$
\left[\lambda+\rho\left(x_{k}\right) \phi_{u_{x_{k}}}\left(x_{k}\right)\right] u_{k}\left(x_{k}\right) \leq u_{k}^{p}\left(x_{k}\right)
$$

and so

$$
u_{k}\left(x_{k}\right) \geq\left[\lambda+\rho\left(x_{k}\right) \phi_{u_{x_{k}}}\left(x_{k}\right)\right]^{\frac{1}{p-1}} \geq \lambda^{\frac{1}{p-1}}>0 .
$$

Therefore, the local maximum values of $u_{k}$, and hence of $w_{k}$, are greater than or equal to $\lambda^{\frac{1}{p-1}}$, and since $w_{k} \rightarrow w_{0}$ in $C_{\text {loc }}^{2}\left(\mathbb{R}^{3}\right)$, then $w_{0} \not \equiv 0$. In particular, this and (6.4), imply that $w_{0}>0$ by the strong maximum principle.

Claim 3. For large $k$, it holds that $\int_{\mathbb{R}^{3}} \int_{\mathbb{R}^{3}} \frac{w_{k}^{2}(y) \rho\left(x_{0}+\epsilon_{k} y\right) w_{k}^{2}(x) \nabla \rho\left(x_{0}+\epsilon_{k} x\right)}{4 \pi|x-y|} \mathrm{d} y \mathrm{~d} x=0$.

We first recall that $w_{k}$, as defined in Claim 2, solves (6.2). Multiplying the first equation in (6.2) by $\nabla w_{k}$ and integrating on $B_{R}(0)$, we get

$$
\begin{aligned}
0= & \int_{B_{R}} \Delta w_{k} \nabla w_{k} \mathrm{~d} x-\int_{B_{R}} \lambda \frac{\nabla w_{k}^{2}}{2} \mathrm{~d} x-\frac{1}{2} \int_{B_{R}} \nabla\left(\rho\left(x_{0}+\epsilon_{k} x\right) \phi_{u_{k}}\left(x_{0}+\epsilon_{k} x\right) w_{k}^{2}\right) \mathrm{d} x \\
& +\frac{\epsilon_{k}}{2} \int_{B_{R}} \rho\left(x_{0}+\epsilon_{k} x\right) \nabla \phi_{u_{k}}\left(x_{0}+\epsilon_{k} x\right) w_{k}^{2} \mathrm{~d} x+\frac{\epsilon_{k}}{2} \int_{B_{R}} \nabla \rho\left(x_{0}+\epsilon_{k} x\right) \phi_{u_{k}}\left(x_{0}+\epsilon_{k} x\right) w_{k}^{2} \mathrm{~d} x \\
& +\int_{B_{R}} \frac{\nabla w_{k}^{p+1}}{p+1} \mathrm{~d} x .
\end{aligned}
$$

By using the divergence theorem and rearranging terms, this becomes

$$
\begin{aligned}
\frac{\epsilon_{k}}{2} \int_{B_{R}} \nabla & \rho\left(x_{0}+\epsilon_{k} x\right) \phi_{u_{k}}\left(x_{0}+\epsilon_{k} x\right) w_{k}^{2} \mathrm{~d} x \\
= & \int_{\partial B_{R}}\left(\lambda \frac{w_{k}^{2}}{2} \nu-\frac{w_{k}^{p+1}}{p+1} \nu+\frac{1}{2} \rho\left(x_{0}+\epsilon_{k} x\right) \phi_{u_{k}}\left(x_{0}+\epsilon_{k} x\right) w_{k}^{2} \nu\right) \mathrm{d} \sigma \\
& \quad-\frac{\epsilon_{k}}{2} \int_{B_{R}} \rho\left(x_{0}+\epsilon_{k} x\right) \nabla \phi_{u_{k}}\left(x_{0}+\epsilon_{k} x\right) w_{k}^{2} \mathrm{~d} x-\int_{B_{R}} \Delta w_{k} \nabla w_{k} \mathrm{~d} x
\end{aligned}
$$

where $\nu$ is the exterior normal field on $B_{R}$. We now focus on the second integral on the righthand side of this equality. We begin by noting that if we multiply the second equation in (6.2) by $\nabla \phi_{u_{k}}\left(x_{0}+\epsilon_{k} x\right)$ and integrate on $B_{R}(0)$, we get

$$
-\int_{B_{R}} \rho\left(x_{0}+\epsilon_{k} x\right) \nabla \phi_{u_{k}}\left(x_{0}+\epsilon_{k} x\right) w_{k}^{2} \mathrm{~d} x=\int_{B_{R}} \Delta \phi_{u_{k}}\left(x_{0}+\epsilon_{k} x\right) \nabla \phi_{u_{k}}\left(x_{0}+\epsilon_{k} x\right) \mathrm{d} x .
$$

Moreover, using the divergence theorem, we see that 


$$
\begin{aligned}
\frac{\epsilon_{k}}{2} \int_{B_{R}} \Delta \phi_{u_{k}}\left(x_{0}+\epsilon_{k} x\right) \frac{\partial}{\partial x_{i}} \phi_{u_{k}}\left(x_{0}+\epsilon_{k} x\right) d x= & \frac{1}{2} \int_{B_{R}} \operatorname{div}\left(\nabla \phi_{u_{k}}\left(x_{0}+\epsilon_{k} x\right) \frac{\partial}{\partial x_{i}} \phi_{u_{k}}\left(x_{0}+\epsilon_{k} x\right)\right) \mathrm{d} x \\
& -\frac{1}{2} \int_{B_{R}} \nabla \phi_{u_{k}}\left(x_{0}+\epsilon_{k} x\right) \frac{\partial}{\partial x_{i}}\left(\nabla \phi_{u_{k}}\left(x_{0}+\epsilon_{k} x\right)\right) \mathrm{d} x \\
= & \frac{1}{2} \int_{\partial B_{R}}\left(\frac{\partial \phi_{u_{k}}\left(x_{0}+\epsilon_{k} x\right)}{\partial \nu} \frac{\partial}{\partial x_{i}} \phi_{u_{k}}\left(x_{0}+\epsilon_{k} x\right)\right. \\
& \left.-\frac{1}{2}\left|\nabla \phi_{u_{k}}\left(x_{0}+\epsilon_{k} x\right)\right|^{2} \nu_{i}\right) \mathrm{d} \sigma .
\end{aligned}
$$

Therefore, combining (6.7) and (6.8), we obtain

$$
\begin{aligned}
-\frac{\epsilon_{k}}{2} \int_{B_{R}} \rho\left(x_{0}+\epsilon_{k} x\right) & \nabla \phi_{u_{k}}\left(x_{0}+\epsilon_{k} x\right) w_{k}^{2} \mathrm{~d} x \\
& =\frac{1}{2} \int_{\partial B_{R}}\left(\nabla \phi_{u_{k}}\left(x_{0}+\epsilon_{k} x\right) \frac{\partial \phi_{u_{k}}\left(x_{0}+\epsilon_{k} x\right)}{\partial \nu}-\frac{1}{2}\left|\nabla \phi_{u_{k}}\left(x_{0}+\epsilon_{k} x\right)\right|^{2} \nu\right) \mathrm{d} \sigma .
\end{aligned}
$$

Turning our attention to the third integral on the right-hand side of (6.6) and by arguing in a similar way as above, we can show that

$$
\int_{B_{R}} \Delta w_{k} \nabla w_{k} \mathrm{~d} x=\int_{\partial B_{R}}\left(\nabla w_{k} \frac{\partial w_{k}}{\partial \nu}-\frac{1}{2}\left|\nabla w_{k}\right|^{2} \nu\right) \mathrm{d} \sigma .
$$

Therefore, using (6.9) and (6.10), we see that (6.6) becomes

$$
\begin{aligned}
\frac{\epsilon_{k}}{2} \int_{B_{R}} \nabla \rho\left(x_{0}+\epsilon_{k} x\right) & \phi_{u_{k}}\left(x_{0}+\epsilon_{k} x\right) w_{k}^{2} \mathrm{~d} x \\
& =\int_{\partial B_{R}}\left(\lambda \frac{w_{k}^{2}}{2} \nu-\frac{w_{k}^{p+1}}{p+1} \nu+\frac{1}{2} \rho\left(x_{0}+\epsilon_{k} x\right) \phi_{u_{k}}\left(x_{0}+\epsilon_{k} x\right) w_{k}^{2} \nu\right. \\
& +\frac{1}{2} \nabla \phi_{u_{k}}\left(x_{0}+\epsilon_{k} x\right) \frac{\partial \phi_{u_{k}}\left(x_{0}+\epsilon_{k} x\right)}{\partial \nu}-\frac{1}{4}\left|\nabla \phi_{u_{k}}\left(x_{0}+\epsilon_{k} x\right)\right|^{2} \nu \\
& \left.-\nabla w_{k} \frac{\partial w_{k}}{\partial \nu}+\frac{1}{2}\left|\nabla w_{k}\right|^{2} \nu\right) \mathrm{d} \sigma
\end{aligned}
$$

Call the integral on the right-hand side of this equation $I_{R}$. Then,

$$
\begin{aligned}
\left|I_{R}\right| \leq & \int_{\partial B_{R}}\left(\lambda \frac{w_{k}^{2}}{2}+\frac{w_{k}^{p+1}}{p+1}+\frac{1}{2} \rho\left(x_{0}+\epsilon_{k} x\right) \phi_{u_{k}}\left(x_{0}+\epsilon_{k} x\right) w_{k}^{2}+\frac{1}{2}\left|\nabla \phi_{u_{k}}\left(x_{0}+\epsilon_{k} x\right)\right|^{2}\right. \\
& \left.+\frac{1}{4}\left|\nabla \phi_{u_{k}}\left(x_{0}+\epsilon_{k} x\right)\right|^{2}+\left|\nabla w_{k}\right|^{2}+\frac{1}{2}\left|\nabla w_{k}\right|^{2}\right) \mathrm{d} \sigma \\
\leq & \frac{3}{2} \int_{\partial B_{R}}\left(\lambda w_{k}^{2}+w_{k}^{p+1}+\rho\left(x_{0}+\epsilon_{k} x\right) \phi_{u_{k}}\left(x_{0}+\epsilon_{k} x\right) w_{k}^{2}+\left|\nabla \phi_{u_{k}}\left(x_{0}+\epsilon_{k} x\right)\right|^{2}+\left|\nabla w_{k}\right|^{2}\right) \mathrm{d} \sigma .
\end{aligned}
$$


So,

$$
\begin{aligned}
\int_{0}^{+\infty}\left|I_{R}\right| \leq \int_{0}^{+\infty} \frac{3}{2} \int_{\partial B_{R}}\left(\lambda w_{k}^{2}+w_{k}^{p+1}+\rho\left(x_{0}+\epsilon_{k} x\right) \phi_{u_{k}}\left(x_{0}+\epsilon_{k} x\right) w_{k}^{2}\right. \\
\left.\quad+\left|\nabla \phi_{u_{k}}\left(x_{0}+\epsilon_{k} x\right)\right|^{2}+\left|\nabla w_{k}\right|^{2}\right) \mathrm{d} \sigma \mathrm{d} R \\
=\frac{3}{2} \int_{\mathbb{R}^{3}}\left(\lambda w_{k}^{2}+w_{k}^{p+1}+\rho\left(x_{0}+\epsilon_{k} x\right) \phi_{u_{k}}\left(x_{0}+\epsilon_{k} x\right) w_{k}^{2}\right. \\
\left.\quad+\left|\nabla \phi_{u_{k}}\left(x_{0}+\epsilon_{k} x\right)\right|^{2}+\left|\nabla w_{k}\right|^{2}\right) \mathrm{d} x \\
<+\infty \text { for each } k
\end{aligned}
$$

since $w_{k}$ is a solution to (6.2). Thus, for each fixed $k$, there exists a sequence $R_{m} \rightarrow+\infty$ as $m \rightarrow+\infty$ such that $I_{R_{m}} \rightarrow 0$ as $m \rightarrow+\infty$. Letting $R=R_{m} \rightarrow+\infty$ in (6.11) yields

$$
\begin{aligned}
0 & =\frac{\epsilon_{k}}{2} \int_{\mathbb{R}^{3}} \nabla \rho\left(x_{0}+\epsilon_{k} x\right) \phi_{u_{k}}\left(x_{0}+\epsilon_{k} x\right) w_{k}^{2} \mathrm{~d} x \\
& =\frac{\epsilon_{k}}{2} \int_{\mathbb{R}^{3}} \int_{\mathbb{R}^{3}} \frac{\epsilon_{k}^{2} w_{k}^{2}(y) \rho\left(x_{0}+\epsilon_{k} y\right) w_{k}^{2}(x) \nabla \rho\left(x_{0}+\epsilon_{k} x\right)}{4 \pi|x-y|} \mathrm{d} y \mathrm{~d} x .
\end{aligned}
$$

Since this holds for each fixed $k$, we have

$$
\int_{\mathbb{R}^{3}} \int_{\mathbb{R}^{3}} \frac{w_{k}^{2}(y) \rho\left(x_{0}+\epsilon_{k} y\right) w_{k}^{2}(x) \nabla \rho\left(x_{0}+\epsilon_{k} x\right)}{4 \pi|x-y|} \mathrm{d} y \mathrm{~d} x=0 .
$$

Claim 4. There exists $R_{0}>0$ and $C>0$ such that, for $k$ sufficiently large, $w_{k}(x) \leq C|x|^{-1} e^{-\frac{\sqrt{\lambda}}{2}|x|}$ for all $|x| \geq R_{0}$.

We first note that, by the concentration assumption, it holds that $w_{k} \rightarrow 0$ as $|x| \rightarrow+\infty$. Namely, there exists $R_{0}>0, K>0$ such that

$$
w_{k} \leq\left(\frac{\lambda}{2}\right)^{\frac{1}{p-1}}, \quad \forall|x| \geq R_{0}, \forall k \geq K
$$

It follows that

$$
w_{k}^{p} \leq \frac{\lambda}{2} w_{k}, \quad \forall|x| \geq R_{0}, \forall k \geq K
$$

and therefore, since $w_{k}$ solves (6.2), we have, for all $|x| \geq R_{0}$ and for all $k \geq K$,

$$
-\Delta w_{k}+\lambda w_{k} \leq-\Delta w_{k}+\left(\lambda+\rho\left(x_{0}+\epsilon_{k} x\right) \phi_{u_{k}}\left(x_{0}+\epsilon_{k} x\right)\right) w_{k}=w_{k}^{p} \leq \frac{\lambda}{2} w_{k}
$$


Namely, it holds that

$$
-\Delta w_{k} \leq-\frac{\lambda}{2} w_{k}, \quad \forall|x| \geq R_{0}, \quad \forall k \geq K
$$

Now, define

$$
\omega(x):=C|x|^{-1} e^{-\frac{\sqrt{\lambda}}{2}|x|}, \quad \text { where } C:=\left(\frac{\lambda}{2}\right)^{\frac{1}{p-1}} R_{0} e^{\frac{\sqrt{\lambda}}{2} R_{0}},
$$

Then, using this definition and (6.13), we see that

$$
w_{k}(x) \leq\left(\frac{\lambda}{2}\right)^{\frac{1}{p-1}}=\omega(x), \quad \text { for }|x|=R_{0}, \quad \forall k \geq K .
$$

It can also be checked that,

$$
\Delta \omega \leq \frac{\lambda}{4} \omega, \quad \text { for }|x| \neq 0
$$

We then define $\bar{\omega}_{k}(x):=w_{k}(x)-\omega(x)$. By (6.16) it holds that

$$
\bar{\omega}_{k}(x) \leq 0, \quad \text { for }|x|=R_{0}, \forall k \geq K \text {. }
$$

Moreover, using (6.15) and (6.17), it holds that

$$
-\Delta \bar{\omega}_{k}+\frac{\lambda}{2} \bar{\omega}_{k} \leq 0, \quad \forall|x| \geq R_{0}, \forall k \geq K
$$

and

$$
\lim _{|x| \rightarrow+\infty} \bar{\omega}_{k}(x)=0
$$

Thus, by the maximum principle on unbounded domains (see e.g. [11]), it follows that,

$$
w_{k}(x) \leq C|x|^{-1} e^{-\frac{\sqrt{\lambda}}{2}|x|}, \quad \forall|x| \geq R_{0},
$$

for $k$ sufficiently large.

Claim 5. $\rho\left(x_{0}\right) \nabla \rho\left(x_{0}\right)=0$.

We first pick a uniform large constant $C>0$ such that for all $x \in \mathbb{R}^{3}$ and large $k$ it holds that

$$
w_{k}(x) \leq \tilde{w}(x):=C(1+|x|)^{-1} e^{-\frac{\sqrt{\lambda}}{2}|x|} .
$$


We now highlight the fact that due to the concentration assumption, from now on, we can take $k$ large enough and a suitable $\epsilon_{1}>0$ such that

$$
\epsilon_{k}<\epsilon_{1}<\min \left\{\epsilon_{0}, \frac{\sqrt{\lambda}}{b}\right\}, \quad \text { if } b>0,
$$

and simply

$$
\epsilon_{k}<\epsilon_{0}, \quad \text { if } b \leq 0 \text {, }
$$

where $\epsilon_{0}>0$ is defined in the statement of the theorem. We assume that $b>0$ as the case $b \leq 0$ is easier and requires only obvious modifications. By the growth assumption on $\rho$, there exists a uniform constant $C_{1}>0$ such that for all $x \in \mathbb{R}^{3}$,

$$
\left|\nabla \rho\left(x_{0}+\epsilon_{k} x\right)\right| \leq C_{1}(1+|x|)^{a} e^{b \epsilon_{1}|x|}=: g(x) .
$$

By the mean value theorem we have

$$
\left|\rho\left(x_{0}+\epsilon_{k} y\right)\right| \leq\left|\epsilon_{k} y\right|\left|\nabla \rho\left(x_{0}+\theta\left(\epsilon_{k} y\right)\right)\right|+\left|\rho\left(x_{0}\right)\right|,
$$

for some $\theta \in[0,1]$. Combining this with the estimate on $\left|\nabla \rho\left(x_{0}+\theta\left(\epsilon_{k} y\right)\right)\right|$, it follows that for some uniform constant $C_{2}>0$ and for all $y \in \mathbb{R}^{3}$,

$$
\left|\rho\left(x_{0}+\epsilon_{k} y\right)\right| \leq C_{2}|y|(1+|y|)^{a} e^{b \epsilon_{1}|y|}+\left|\rho\left(x_{0}\right)\right|=: f(y)
$$

Therefore, putting everything together, we have that, for $k$ sufficiently large,

$$
\left|\frac{w_{k}^{2}(y) \rho\left(x_{0}+\epsilon_{k} y\right) w_{k}^{2}(x) \nabla \rho\left(x_{0}+\epsilon_{k} x\right)}{(x-y)}\right| \leq \frac{\tilde{w}^{2}(y) f(y) \tilde{w}^{2}(x) g(x)}{|x-y|} .
$$

The right hand side is a uniform $L^{1}\left(\mathbb{R}^{6}\right)$ bound. In fact, using for instance the Hardy-LittlewoodSobolev inequality, we have

$$
\begin{aligned}
\left|\int_{\mathbb{R}^{3}} \int_{\mathbb{R}^{3}} \frac{\tilde{w}^{2}(y) f(y) \tilde{w}^{2}(x) g(x)}{|x-y|} \mathrm{d} y \mathrm{~d} x\right| & \lesssim\left\|\tilde{w}^{2} f\right\|_{L^{6 / 5}\left(\mathbb{R}^{3}\right)}\left\|\tilde{w}^{2} g\right\|_{L^{6 / 5}\left(\mathbb{R}^{3}\right)} \\
& <+\infty
\end{aligned}
$$

as the choice of $\epsilon_{1}$ implies that $\tilde{w}^{2} f, \tilde{w}^{2} g \in L^{6 / 5}\left(\mathbb{R}^{3}\right)$. We now let $k \rightarrow+\infty$ in (6.12), and note that by (6.22), (6.23), Claim 2 , and the assumption that $\rho \in C^{1}\left(\mathbb{R}^{3}\right)$, we can use the dominated convergence theorem to obtain

$$
\int_{\mathbb{R}^{3}} \int_{\mathbb{R}^{3}} \frac{w_{0}^{2}(y) \rho\left(x_{0}\right) w_{0}^{2}(x) \nabla \rho\left(x_{0}\right)}{4 \pi|x-y|} \mathrm{d} y \mathrm{~d} x=0 .
$$

Then, since $w_{0}>0$ by Claim 2, we have that

$$
\rho\left(x_{0}\right) \nabla \rho\left(x_{0}\right)=0 .
$$


Since $\rho$ is nonnegative, any zero is global minimum, and therefore we have $\nabla \rho\left(x_{0}\right)=0$.

6.2. Proof of Theorem 6. We follow up Theorem 5 with a similar result on necessary conditions for concentration of solutions in $H^{1}\left(\mathbb{R}^{3}\right) \times D^{1,2}\left(\mathbb{R}^{3}\right)$.

Proof of Theorem 6. The proof closely follows that of Theorem 5. We assert that the same five claims as were made in the proof of Theorem 5 hold, and will only highlight the differences in the proofs of these claims. The proof of Claim 1 and Claim 2 follow similarly as in Theorem 5 , however since $\rho$ is both continuous and globally bounded in this case, we do not need to fix a specific compact set $K$ in the regularity arguments, but instead it follows directly that $v_{m}$ is uniformly bounded in $C_{\text {loc }}^{2, \alpha}\left(\mathbb{R}^{3}\right)$ and $w_{k}$ is uniformly bounded in $C_{\text {loc }}^{2, \alpha}\left(\mathbb{R}^{3}\right)$. The proof of Claim 3 and Claim 4 follow exactly as in Theorem 5. To prove Claim 5, we define the exponentially decaying function $\tilde{w}$ as in (6.21) and since $\rho$ and $\nabla \rho$ are bounded, we have, for $k$ sufficiently large,

$$
\left|\frac{w_{k}^{2}(y) \rho\left(x_{0}+\epsilon_{k} y\right) w_{k}^{2}(x) \nabla \rho\left(x_{0}+\epsilon_{k} x\right)}{(x-y)}\right| \lesssim \frac{\tilde{w}^{2}(y) \tilde{w}^{2}(x)}{|x-y|} \in L^{1}\left(\mathbb{R}^{6}\right) .
$$

This is enough to conclude the proof as in Theorem 5 using the dominated convergence theorem.

\section{APPEndix: PROOF OF LEMMA 2.2}

Proof of Lemma 2.2. With the regularity remarks of Section 2.2 in place, we now multiply the first equation in $(2.1)$ by $(x, \nabla u)$ and integrate on $B_{R}(0)$ for some $R>0$. We will compute each integral separately. We first note that by Lemma 3.1 in [22] it holds that

$$
\int_{B_{R}}-\Delta u(x, \nabla u) \mathrm{d} x=-\frac{1}{2} \int_{B_{R}}|\nabla u|^{2} \mathrm{~d} x-\frac{1}{R} \int_{\partial B_{R}}|(x, \nabla u)|^{2} \mathrm{~d} \sigma+\frac{R}{2} \int_{\partial B_{R}}|\nabla u|^{2} \mathrm{~d} \sigma .
$$

Fixing $i=1,2,3$, integrating by parts and using the divergence theorem, we then see that,

$$
\begin{aligned}
\int_{B_{R}} b u\left(x_{i} \partial_{i} u\right) \mathrm{d} x & =b\left[-\frac{1}{2} \int_{B_{R}} u^{2} \mathrm{~d} x+\frac{1}{2} \int_{B_{R}} \partial_{i}\left(u^{2} x_{i}\right) \mathrm{d} x\right] \\
& =b\left[-\frac{1}{2} \int_{B_{R}} u^{2} \mathrm{~d} x+\frac{1}{2} \int_{\partial B_{R}} u^{2} \frac{x_{i}^{2}}{|x|} \mathrm{d} \sigma\right]
\end{aligned}
$$

So, summing over $i$, we get

$$
\int_{B_{R}} b u(x, \nabla u) \mathrm{d} x=b\left[-\frac{3}{2} \int_{B_{R}} u^{2} \mathrm{~d} x+\frac{R}{2} \int_{\partial B_{R}} u^{2} \mathrm{~d} \sigma\right] .
$$

Again, fixing $i=1,2,3$, integrating by parts and using the divergence theorem, we find that,

$$
\begin{aligned}
\int_{B_{R}} c \rho \phi_{u} u x_{i}\left(\partial_{i} u\right) \mathrm{d} x=c[- & \frac{1}{2} \int_{B_{R}} \rho \phi_{u} u^{2} \mathrm{~d} x-\frac{1}{2} \int_{B_{R}} \phi_{u} u^{2} x_{i}\left(\partial_{i} \rho\right) \mathrm{d} x-\frac{1}{2} \int_{B_{R}} \rho u^{2} x_{i}\left(\partial_{i} \phi_{u}\right) \mathrm{d} x \\
& \left.+\frac{1}{2} \int_{B_{R}} \partial_{i}\left(\rho \phi_{u} u^{2} x_{i}\right) \mathrm{d} x\right]
\end{aligned}
$$




$$
\begin{aligned}
=c\left[-\frac{1}{2} \int_{B_{R}} \rho \phi_{u} u^{2} \mathrm{~d} x-\frac{1}{2} \int_{B_{R}} \phi_{u} u^{2} x_{i}\left(\partial_{i} \rho\right) \mathrm{d} x-\frac{1}{2} \int_{B_{R}} \rho u^{2} x_{i}\left(\partial_{i} \phi_{u}\right) \mathrm{d} x\right. \\
\left.+\frac{1}{2} \int_{\partial B_{R}} \rho \phi_{u} u^{2} \frac{x_{i}^{2}}{|x|} \mathrm{d} \sigma\right] .
\end{aligned}
$$

Thus, summing over $i$, we get

$$
\begin{aligned}
\int_{B_{R}} c \rho \phi_{u} u(x, \nabla u) \mathrm{d} x=c[- & \frac{3}{2} \int_{B_{R}} \rho \phi_{u} u^{2} \mathrm{~d} x-\frac{1}{2} \int_{B_{R}} \phi_{u} u^{2}(x, \nabla \rho) \mathrm{d} x \\
& \left.-\frac{1}{2} \int_{B_{R}} \rho u^{2}\left(x, \nabla \phi_{u}\right) \mathrm{d} x+\frac{R}{2} \int_{\partial B_{R}} \rho \phi_{u} u^{2} \mathrm{~d} \sigma\right] .
\end{aligned}
$$

Finally, once more fixing $i=1,2,3$, integrating by parts and using the divergence theorem, we find that,

$$
\int_{B_{R}} d|u|^{p-1} u\left(x_{i} \partial_{i} u\right) \mathrm{d} x=d\left[\frac{-1}{p+1} \int_{B_{R}}|u|^{p+1} \mathrm{~d} x+\frac{1}{p+1} \int_{\partial B_{R}}|u|^{p+1} \frac{x_{i}^{2}}{|x|} \mathrm{d} \sigma\right],
$$

and so, summing over $i$, we see that

$$
\int_{B_{R}} d|u|^{p-1} u(x, \nabla u) \mathrm{d} x=d\left[\frac{-3}{p+1} \int_{B_{R}}|u|^{p+1} \mathrm{~d} x+\frac{R}{p+1} \int_{\partial B_{R}}|u|^{p+1} \mathrm{~d} \sigma\right]
$$

Putting (6.24), (6.25), (6.26) and (6.27) together, we see that

$$
\begin{aligned}
- & \frac{1}{2} \int_{B_{R}}|\nabla u|^{2} \mathrm{~d} x-\frac{1}{R} \int_{\partial B_{R}}|(x, \nabla u)|^{2} \mathrm{~d} \sigma+\frac{R}{2} \int_{\partial B_{R}}|\nabla u|^{2} \mathrm{~d} \sigma+b\left[-\frac{3}{2} \int_{B_{R}} u^{2} \mathrm{~d} x\right. \\
& \left.+\frac{R}{2} \int_{\partial B_{R}} u^{2} \mathrm{~d} \sigma\right]+c\left[-\frac{3}{2} \int_{B_{R}} \rho \phi_{u} u^{2} \mathrm{~d} x-\frac{1}{2} \int_{B_{R}} \phi_{u} u^{2}(x, \nabla \rho) \mathrm{d} x-\frac{1}{2} \int_{B_{R}} \rho u^{2}\left(x, \nabla \phi_{u}\right) \mathrm{d} x\right.
\end{aligned}
$$

$$
\left.+\frac{R}{2} \int_{\partial B_{R}} \rho \phi_{u} u^{2} \mathrm{~d} \sigma\right]-d\left[\frac{-3}{p+1} \int_{B_{R}}|u|^{p+1} \mathrm{~d} x+\frac{R}{p+1} \int_{\partial B_{R}}|u|^{p+1} \mathrm{~d} \sigma\right]=0 .
$$

We now multiply the second equation in $(2.1)$ by $\left(x, \nabla \phi_{u}\right)$ and integrate on $B_{R}(0)$ for some $R>0$. Using Lemma 3.1 in [22] we see that

$$
\begin{aligned}
\int_{B_{R}} \rho u^{2}\left(x, \nabla \phi_{u}\right) \mathrm{d} x & =\int_{B_{R}}-\Delta \phi_{u}\left(x, \nabla \phi_{u}\right) \mathrm{d} x \\
& =-\frac{1}{2} \int_{B_{R}}\left|\nabla \phi_{u}\right|^{2} \mathrm{~d} x-\frac{1}{R} \int_{\partial B_{R}}\left|\left(x, \nabla \phi_{u}\right)\right|^{2} \mathrm{~d} \sigma+\frac{R}{2} \int_{\partial B_{R}}\left|\nabla \phi_{u}\right|^{2} \mathrm{~d} \sigma .
\end{aligned}
$$

Substituting this into (6.28) and rearranging, we get 


$$
\begin{aligned}
&-\frac{1}{2} \int_{B_{R}}|\nabla u|^{2} \mathrm{~d} x-\frac{3 b}{2} \int_{B_{R}} u^{2} \mathrm{~d} x-\frac{3 c}{2} \int_{B_{R}} \rho \phi_{u} u^{2} \mathrm{~d} x \\
&- \frac{c}{2} \int_{B_{R}} \phi_{u} u^{2}(x, \nabla \rho) \mathrm{d} x+\frac{c}{4} \int_{B_{R}}\left|\nabla \phi_{u}\right|^{2} \mathrm{~d} x+\frac{3 d}{p+1} \int_{B_{R}}|u|^{p+1} \mathrm{~d} x \\
&=\frac{1}{R} \int_{\partial B_{R}}|(x, \nabla u)|^{2} \mathrm{~d} \sigma-\frac{R}{2} \int_{\partial B_{R}}|\nabla u|^{2} \mathrm{~d} \sigma-\frac{b R}{2} \int_{\partial B_{R}} u^{2} \mathrm{~d} \sigma-\frac{c R}{2} \int_{\partial B_{R}} \rho \phi_{u} u^{2} \mathrm{~d} \sigma \\
& \quad-\frac{c}{2 R} \int_{\partial B_{R}}\left|\left(x, \nabla \phi_{u}\right)\right|^{2} \mathrm{~d} \sigma+\frac{c R}{4} \int_{\partial B_{R}}\left|\nabla \phi_{u}\right|^{2} \mathrm{~d} \sigma+\frac{d R}{p+1} \int_{\partial B_{R}}|u|^{p+1} \mathrm{~d} \sigma .
\end{aligned}
$$

We now call the right hand side of this equality $I_{R}$. We note that $|(x, \nabla u)| \leq R|\nabla u|$ and $\left|\left(x, \nabla \phi_{u}\right)\right| \leq R\left|\nabla \phi_{u}\right|$ on $\partial B_{R}$, so it holds that

$$
\begin{aligned}
\left|I_{R}\right| \leq & \frac{3 R}{2} \int_{\partial B_{R}}|\nabla u|^{2} \mathrm{~d} \sigma+\frac{b R}{2} \int_{\partial B_{R}} u^{2} \mathrm{~d} \sigma \\
& +\frac{c R}{2} \int_{\partial B_{R}} \rho \phi_{u} u^{2} \mathrm{~d} \sigma+\frac{3 c R}{4} \int_{\partial B_{R}}\left|\nabla \phi_{u}\right|^{2} \mathrm{~d} \sigma+\frac{d R}{p+1} \int_{\partial B_{R}}|u|^{p+1} \mathrm{~d} \sigma .
\end{aligned}
$$

Now, since $|\nabla u|^{2}, u^{2} \in L^{1}\left(\mathbb{R}^{3}\right)$ because $u \in E\left(\mathbb{R}^{3}\right) \subset H^{1}\left(\mathbb{R}^{3}\right), \rho \phi_{u} u^{2},\left|\nabla \phi_{u}\right|^{2} \in L^{1}\left(\mathbb{R}^{3}\right)$ because $\int_{\mathbb{R}^{3}} \rho \phi_{u} u^{2} \mathrm{~d} x=\int_{\mathbb{R}^{3}}\left|\nabla \phi_{u}\right|^{2} \mathrm{~d} x$ and $\phi_{u} \in D^{1,2}\left(\mathbb{R}^{3}\right)$, and $|u|^{p+1} \in L^{1}\left(\mathbb{R}^{3}\right)$ because $E\left(\mathbb{R}^{3}\right) \hookrightarrow L^{q}\left(\mathbb{R}^{3}\right)$ for all $q \in[2,6]$, then it holds that $I_{R_{n}} \rightarrow 0$ as $n \rightarrow+\infty$ for a suitable sequence $R_{n} \rightarrow+\infty$ (see e.g. [22]). Thus, considering (6.29) with $R=R_{n}$, we see that

$$
\begin{aligned}
-\frac{c}{2} \int_{\mathbb{R}^{3}} \phi_{u} u^{2}(x, \nabla \rho) \mathrm{d} x & \lim _{n \rightarrow+\infty}\left(-\frac{c}{2} \int_{B_{R_{n}}} \phi_{u} u^{2}(x, \nabla \rho) \mathrm{d} x\right) \\
= & \lim _{n \rightarrow+\infty}\left(\frac{1}{2} \int_{B_{R_{n}}}|\nabla u|^{2} \mathrm{~d} x+\frac{3 b}{2} \int_{B_{R_{n}}} u^{2} \mathrm{~d} x+\frac{3 c}{2} \int_{B_{R_{n}}} \rho \phi_{u} u^{2} \mathrm{~d} x-\frac{c}{4} \int_{B_{R}}\left|\nabla \phi_{u}\right|^{2} \mathrm{~d} x\right. \\
& \quad-\frac{3 d}{p+1} \int_{B_{R_{n}}}|u|^{p+1} \mathrm{~d} x+\frac{1}{R_{n}} \int_{\partial B_{R_{n}}}|(x, \nabla u)|^{2} \mathrm{~d} \sigma-\frac{R_{n}}{2} \int_{\partial B_{R_{n}}}|\nabla u|^{2} \mathrm{~d} \sigma \\
& \quad-\frac{b R_{n}}{2} \int_{\partial B_{R_{n}}} u^{2} \mathrm{~d} \sigma-\frac{c R_{n}}{2} \int_{\partial B_{R_{n}}} \rho \phi_{u} u^{2} \mathrm{~d} \sigma-\frac{c}{2 R_{n}} \int_{\partial B_{R_{n}}}\left|\left(x, \nabla \phi_{u}\right)\right|^{2} \mathrm{~d} \sigma \\
=\frac{1}{2} & \int_{\mathbb{R}^{3}}|\nabla u|^{2} \mathrm{~d} x+\frac{3 b}{2} \int_{\mathbb{R}^{3}} u^{2} \mathrm{~d} x+\frac{3 c}{2} \int_{\mathbb{R}^{3}} \rho \phi_{u} u^{2} \mathrm{~d} x \\
& -\frac{c}{4} \int_{\mathbb{R}^{3}}\left|\nabla \phi_{u}\right|^{2} \mathrm{~d} x-\frac{d R_{n}}{p+1} \int_{\mathbb{R}^{3}}|u|^{p+1} \mathrm{~d} x \\
= & \int_{\mathbb{R}^{3}}|\nabla u|^{2} \mathrm{~d} x+\frac{3 b}{2} \int_{\mathbb{R}^{3}} u^{2} \mathrm{~d} x+\frac{5 c}{4} \int_{\mathbb{R}^{3}} \rho \phi_{u} u^{2} \mathrm{~d} x-\frac{3 d}{p+1} \int_{\mathbb{R}^{3}}|u|^{p+1} \mathrm{~d} x
\end{aligned}
$$


where the final equality holds because $\int_{\mathbb{R}^{3}}\left|\nabla \phi_{u}\right|^{2} \mathrm{~d} x=\int_{\mathbb{R}^{3}} \rho \phi_{u} u^{2} \mathrm{~d} x$. Therefore, since $\left(u, \phi_{u}\right) \in$ $E\left(\mathbb{R}^{3}\right) \times D^{1,2}\left(\mathbb{R}^{3}\right)$ solves $(2.1)$, we have shown that

$$
\left|\frac{c}{2} \int_{\mathbb{R}^{3}} \phi_{u} u^{2}(x, \nabla \rho) \mathrm{d} x\right|<+\infty
$$

Moreover, (6.30) also proves that

$$
\frac{1}{2} \int_{\mathbb{R}^{3}}|\nabla u|^{2}+\frac{3 b}{2} \int_{\mathbb{R}^{3}} u^{2}+\frac{5 c}{4} \int_{\mathbb{R}^{3}} \rho \phi_{u} u^{2}+\frac{c}{2} \int_{\mathbb{R}^{3}}(x, \nabla \rho) u^{2} \phi_{u}-\frac{3 d}{p+1} \int_{\mathbb{R}^{3}}|u|^{p+1}=0 .
$$

\section{REFERENCES}

[1] R. Adams. Sobolev Spaces. New York, NY: Academic Press, 1975.

[2] A. Ambrosetti. On Schrödinger- Poisson systems. Milan J. Math., 2008, 76, pp. 257-274.

[3] A. Ambrosetti and A. Malchiodi. Perturbation Methods and Semilinear Elliptic Problems on $\mathbb{R}^{n}$. Progress in Mathematics, 240. Birkhäuser Verlag, Basel, 2006.

[4] A. Ambrosetti and P. H. Rabinowitz. Dual variational methods in critical point theory and its applications. J. Funct. Anal., 1973, 14, pp.349-381.

[5] A. Ambrosetti and D. Ruiz. Multiple bound states for the Schrödinger- Poisson problem. Commun. Contemp. Math, 2008, 10, pp. 391-404.

[6] W. Bao, N. J. Mauser and H. P. Stimming. Effective one particle quantum dynamics of electrons: a numerical study of the Schrödinger-Poisson-X $\alpha$ model. Commun. Math. Sci, 2003, 1(4), pp. 809-828.

[7] J. Bellazzini, R. Frank and N. Visciglia. Maximizers for Gagliardo-Nirenberg inequalities and related non-local problems. Mat. Ann., 2014, 360(3-4), pp. 653-673.

[8] J. Bellazzini, M. Ghimenti, C. Mercuri, V. Moroz and J. Van Schaftingen. Sharp Gagliardo-Nirenberg inequalities in fractional Coulomb-Sobolev spaces. Transactions of AMS, 2018, 370(11), pp. 8285-8310.

[9] V. Benci and G. Cerami. Positive solutions of some nonlinear elliptic problems in exterior domains. Arch. Rat. Mech. Anal., 1987, 99, pp. 283-300 .

[10] V. Benci and D. Fortunato. Variational methods in nonlinear field equations. Solitary waves, hylomorphic solitons and vortices. Springer Monographs in Mathematics. Springer, Cham, 2014.

[11] H. Berestycki, L. Caffarelli and L. Nirenberg. Monotonicity for elliptic equations in unbounded Lipschitz domains. Comm. Pure Appl. Math., 1997, 50, pp. 1089-1111.

[12] O. Bokanowski, J. L. López and J. Soler. On an exchange interaction model for quantum transport: the Schrödinger-Poisson-Slater system. Math. Models Methods Appl. Sci., 2003, 13(10), pp. 1397-1412.

[13] D. Bonheure. Personal communication.

[14] D. Bonheure, J. Di Cosmo and C. Mercuri. Concentration on circles for nonlinear Schrödinger-Poisson systems with unbounded potentials vanishing at infinity. Commun. Contemp. Math., 2012, 14(2), pp. 31.

[15] D. Bonheure and C. Mercuri. Embedding theorems and existence results for nonlinear Schrödinger-Poisson systems with unbounded and vanishing potentials. J. Differential Equations, 2011, 251, pp. 1056-1085.

[16] D. Bonheure and J. Van Schaftingen. Groundstates for the nonlinear Schrödinger equation with potential vanishing at infinity. Ann. Mat. Pura Appl., 2010, 189, pp. 273-301.

[17] H. Brezis and E. Lieb. A relation between pointwise convergence of functions and convergence of functionals. Proc. Am. Math. Soc., 1983, 88(3), pp. 486-490.

[18] I. Catto, J. Dolbeault, O. Sanchez and J. Soler. Existence of steady states for the Maxwell-Schrödinger-Poisson system: exploring the applicability of the concentration-compactness principle. Math. Models Methods Appl. Sci., 2013, 23(10), pp. 1915-1938.

[19] G. Cerami and R. Molle. Multiple positive bound states for critical Schrödinger-Poisson systems. arXiv:1802.02539.

[20] G. Cerami and R. Molle. Positive bound state solutions for some Schrödinger-Poisson systems. Nonlinearity, 2016, 29, pp. 3103-3119.

[21] G. Cerami and G. Vaira. Positive solutions for some non-autonomous Schrödinger-Poisson systems. J. Differential Equations, 2010, 248, pp. 521-543.

[22] T. D'Aprile and D. Mugnai. Non-Existence Results for the Coupled Klein-Gordon-Maxwell Equations. Adv. Nonlinear Stud., 2004, 4(3), pp. 307-322. 
[23] M. Degiovanni and S. Lancelotti. Linking over cones and nontrivial solutions for $p$-Laplace equations with p-superlinear nonlinearity. Ann. Inst. H. Poincaré Anal. Non Linéaire, 2007, 24, pp. 907-919.

[24] M. del Pino and P. Felmer. Local mountain passes for semilinear elliptic problems in unbounded domains. Calc. Var. Partial Differential Equations, 1996, 4(2), pp. 121-137.

[25] L. Evans. Partial differential equations. American Mathematical Society, 1998.

[26] B. Gidas and J. Spruck. A priori bounds for positive solutions of nonlinear elliptic equations. Comm. PDE, 1981, 6, pp. 883-901.

[27] D. Gilbarg and N. Trudinger. Elliptic partial differential equations of second order, $2^{\text {nd }}$ edition. New York, Berlin: Springer, 1983.

[28] I. Ianni and D. Ruiz. Ground and bound states for a static Schrödinger-Poisson-Slater problem. Commun. Contemp. Math., 2012, 14(1), 1250003, 22.

[29] I. Ianni and G. Vaira. On concentration of positive bound states for the Schrödinger-Poisson problem with potentials. Advanced Nonlin. Studies, 2016, 8(3), pp. 573-595.

[30] L. Jeanjean. On the existence of bounded Palais-Smale sequences and applications to a Landesman-Lazer type problem set on $\mathbb{R}^{N}$. Proc. Roy. Soc. Edinburgh, 1999, 129, pp. 787-809.

[31] L. Jeanjean and K. Tanaka. A positive solution for a nonlinear Schrödinger equation on $\mathbb{R}^{N}$. Indiana University Mathematics Journal, 2005, 54(2), pp. 443-464.

[32] W. Jeong and J. Seok. On perturbation of a functional with the mountain pass geometry: applications to the nonlinear Schrödinger-Poisson equations and the nonlinear Klein-Gordon-Maxwell equations. Calc. Var. Partial Differential Equations, 2014, 49(1-2), pp. 649-668.

[33] M. Kwong. Uniqueness of positive solutions of $\Delta u-u+u^{p}=0$ in $\mathbb{R}^{n}$. Arch. Rat. Mech. Anal., 1989, 105, pp. $243-266$.

[34] E. H. Lieb and M. Loss. Analysis, $2^{\text {nd }}$ edition. Rhode Island: American Mathematical Society, 2001.

[35] P. L. Lions. Some remarks on Hartree equation. Nonlinear Anal., 1981, 5(11), pp. 1245-1256.

[36] P. L. Lions. The concentration-compactness principle in the calculus of variations. The locally compact case. Ann. Inst. H. Poincairé Anal. Non Linéaire, 1984, 1, pp. 109-145 and 223-283.

[37] P. L. Lions. Solutions of Hartree-Fock equations for Coulomb systems. Comm. Math. Phys., 1987, 109(1), pp. 33-97.

[38] N. J. Mauser. The Schrödinger-Poisson-X $\alpha$ equation. Appl. Math. Lett., 2001, 14(6), pp. 759-763.

[39] C. Mercuri. Positive solutions of nonlinear Schrödinger-Poisson systems with radial potentials vanishing at infinity. Atti Accad. Naz. Lincei Rend. Lincei Mat. Appl., 2008, 19(3), pp. $211-227$.

[40] C. Mercuri, V. Moroz and J. Van Schaftingen. Groundstates and radial solutions to nonlinear SchrödingerPoisson-Slater equations at the critical frequency. Calc. Var. Partial Differential Equations, 2016, 55(6), pp. $1-58$.

[41] C. Mercuri and M. Squassina. Global compactness for a class of quasi-linear problems. Manuscripta Math., 2013, 140(1-2), pp. 119-144.

[42] C. Mercuri and M. Willem. A global compactness result for the p-Laplacian involving critical nonlinearities. Discrete and Continuous Dynamical Systems, 2010, 28(2), pp. 469-493.

[43] P. Rabinowitz. On a class of nonlinear Schrödinger equations. Z. Angew. Math. Phys., 1992, 43(2), pp. $229-266$.

[44] D. Ruiz. On the Schrödinger-Poisson-Slater system: behavior of minimizers, radial and nonradial cases. Arch. Rat. Mech. Anal., 2010, 198, pp. 349-368.

[45] D. Ruiz. The Schrödinger-Poisson equation under the effect of a nonlinear local term. J. Functional Analysis, 2006, 237, pp. 655-674.

[46] J. Slater. A simplification of the Hartree-Fock Method. Phys. Rev., 1951, 81, pp. 385-390.

[47] M. Struwe. On the evolution of harmonic mappings of Riemannian surfaces. Comment. Math. Helvetici, 1985, 60, pp. 558-581.

[48] M. Struwe. Variational Methods: Applications to Nonlinear Partial Differential Equations and Hamiltonian Systems, $4^{\text {th }}$ edition. Berlin: Springer-Verlag, 2008.

[49] J. Sun, T. Wu and Z. Feng. Non-autonomous Schrödinger-Poisson System in $\mathbb{R}^{3}$. Discrete and Continuous Dynamical Systems, 2018, 38(4), pp. 1889-1933.

[50] G. Vaira. Ground states for Schrödinger-Poisson type systems. Ricerche mat., 2011, 60, pp. $263-297$.

[51] X. Wang. On concentration of positive bound states of nonlinear Schrödinger equations. Comm. Math. Phys., 1993, 153, pp. 229-224.

[52] M. Willem. Minimax Theorems, Birkhäuser Boston, MA, 1996 
Department of Mathematics, Swansea University, Fabian Way, Crymlyn Burrows, Skewen, Swansea, U.K. SA1 8EN

E-mail address: c.mercuri@swansea.ac.uk

Department of Mathematics, Swansea University, Fabian Way, Crymlyn Burrows, Skewen, Swansea, U.K. SA1 8EN

E-mail address: 807424@swansea.ac.uk 